\title{
Measurements of Wind, Aeolian Sand Transport, and Precipitation in the Colorado River Corridor, Grand Canyon, Arizona - November 2003 to December 2004
}

By Amy E. Draut and David M. Rubin

Any use of trade, firm, or product names is for descriptive purposes only and does not imply endorsement by the U.S. Government

Open-File Report 2005-1309

U.S. Department of the Interior U.S. Geological Survey 


\title{
Measurements of Wind, Aeolian Sand Transport, and Precipitation in the Colorado River Corridor, Grand Canyon, Arizona-November 2003 to December 2004
}

\author{
Amy E. Draut ${ }^{1}$ and David M. Rubin ${ }^{2}$ \\ ${ }^{1}$ US Geological Survey Pacific Sciences Center, Santa Cruz, CA and \\ University of California, Santa Cruz, CA \\ ${ }^{2}$ US Geological Survey Pacific Sciences Center, Santa Cruz, CA
}

U.S. GEOLOGICAL SURVEY

Open-File Report 2005-1309 


\section{U.S. Department of the Interior \\ Gale A. Norton, Secretary}

\section{U.S. Geological Survey \\ Charles G. Groat, Director}

U.S. Geological Survey, Reston, Virginia 2005

Revised and reprinted: 2005

To download a copy of this report from the

World Wide Web: http://pubs.usgs.gov/of/2005/1309/

For more information on the USGS - the Federal source for science about the Earth, its natural and living resources, natural hazards, and the environment:

World Wide Web: http://www.usgs.gov

Telephone: 1-888-ASK-USGS

To contact the authors:

Amy Draut, USGS Pacific Sciences Center, Santa Cruz CA 95060

831-427-4733; adraut@usgs.gov

David Rubin, USGS Pacific Sciences Center, Santa Cruz CA 95060

831-427-4736; drubin@usgs.gov

For more information on USGS science in Grand Canyon, Arizona through the Grand Canyon Monitoring and Research Center: http://www.gcmrc.gov

Although this report is in the public domain, permission must be secured from the individual copyright owners to reproduce any copyrighted material contained within this report. 


\begin{abstract}
This report presents measurements of aeolian sediment-transport rates, wind speed and direction, and precipitation records from nine locations in the Colorado River corridor in Grand Canyon, Grand Canyon National Park, Arizona. Aeolian deposits, many of which contain and preserve archaeological material, are a critical part of the Grand Canyon ecosystem. Data collected between November 2003 and December 2004 indicate that wind velocities and sand transport were greatest during April and May 2004 (with maximum winds locally $>25 \mathrm{~m} \mathrm{~s}^{-1}$, and transport rates locally $\sim 5 \mathrm{~kg} \mathrm{~cm}^{-1}$ day $^{-1}$ ). Dominant wind direction during strong wind intervals varies with location, but during the April-May windy season the greatest transport potential was directed upstream in Marble Canyon (upper Grand Canyon). Such information can be used to evaluate the potential for aeolian reworking of new fluvial sand deposits, and restoration of higherelevation aeolian deposits, following a 60-hour controlled flood release from Glen Canyon Dam in November 2004.
\end{abstract}

\title{
INTRODUCTION
}

In the fall of 2003, a temporary network of weather stations was established in the Colorado River corridor in Grand Canyon, Grand Canyon National Park, Arizona, to monitor wind, precipitation, and aeolian sedimenttransport patterns over an interval of approximately two years. Data collected at these stations constitutes the only continuous weather record from the river corridor with the exception of wind and rainfall measurements made at Phantom Ranch (river mile 88) by the National Park Service. These data allow resolution of seasonal and regional variability in wind intensity and direction, and resultant aeolian sediment transport, as well as precipitation patterns. This report contains data collected between November 2003 and December 2004 at three sites, and data from April through December 2004 collected at three additional sites. 
High-resolution records from these weather stations can be used to identify rainfall events that cause gully incision and to predict aeolian sediment redistribution, aiding other sedimentary and geomorphic studies of sediment deposits in the river corridor. The condition of fluvial and aeolian deposits in the river corridor is of particular concern to scientists and recreational visitors to Grand Canyon National Park, in light of the depletion of sediment deposits since 1963 attributed to sediment-supply limiting effects of Glen Canyon Dam (Beus and others, 1985; Schmidt and Graf, 1987; Kearsley and others, 1994; Kaplinski and others, 1995; Topping and others, 2000a, b; Rubin and Topping, 2001; Rubin and others, 2002; Schmidt and others, 2004). Data collected at these stations during the year before the November 2004 high-flow release from Glen Canyon Dam can, in particular, be used to predict aeolian redistribution of new sediment deposited by that flood. These records are therefore important for assessing the potential of controlled flooding to replenish aeolian sediment deposits above the flood-stage elevation. Many of these aeolian deposits contain and provide a protective cover for archaeological material, a valuable component of the Grand Canyon ecosystem.

\section{Study Sites:}

The six study sites where weather stations were established for this project are shown in figure 1. Locations in the river corridor are commonly referred to by distance in miles downstream of Lees Ferry; this report follows that convention while using metric units for other measurements. Three sites in upper Grand Canyon, at 24.5 mile, Malgosa (river mile 57.9) and Palisades (river mile 66.1) were equipped with two weather stations each, beginning in November 2003. Three additional sites, at Comanche (river mile 68.0), Forster (river mile 123), and 202.9 mile, have been studied since April 2004 with one weather station at each site.

Study sites were selected for instrumentation based on several factors. In order to provide data that will be relevant to monitoring of archaeological 


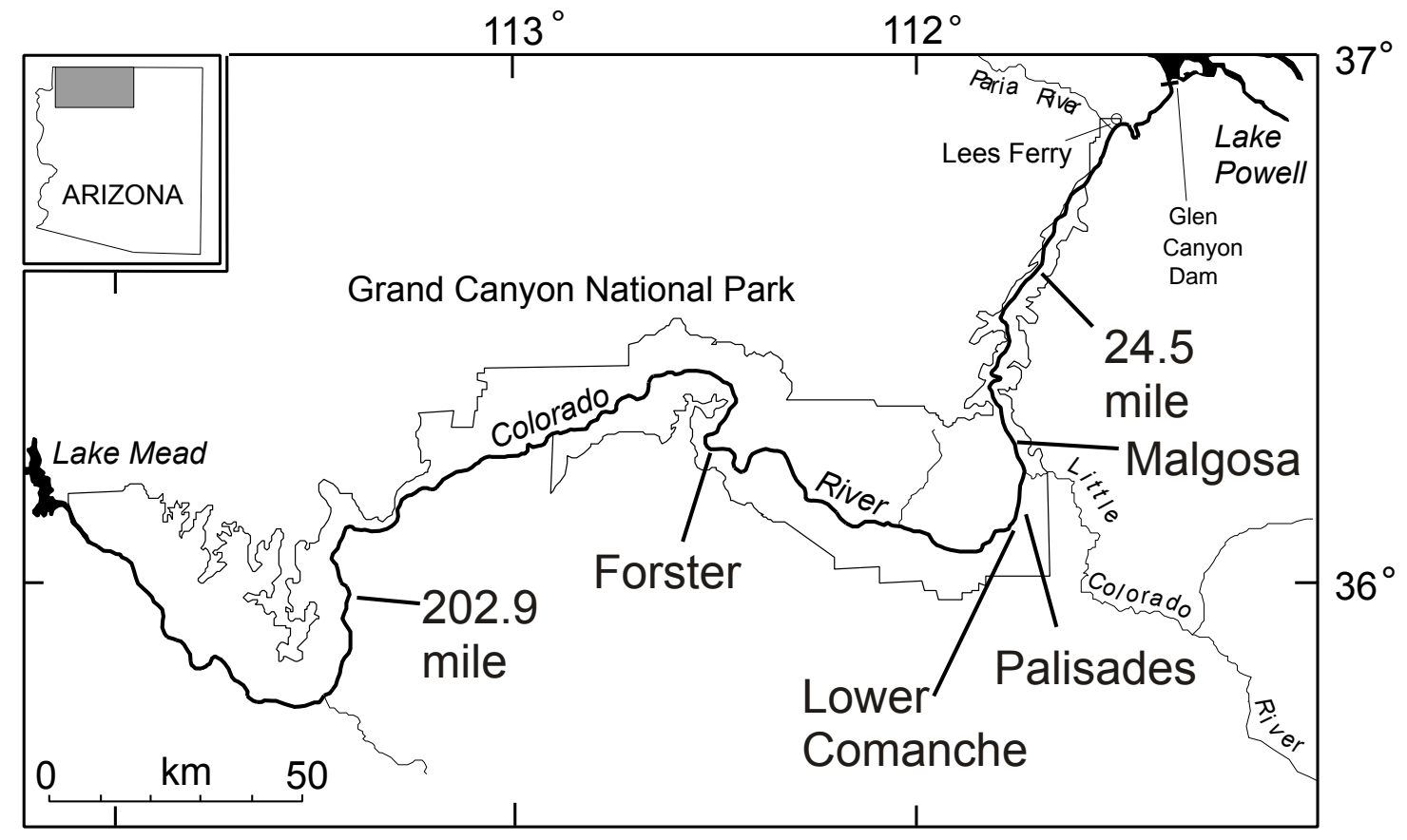

FIGURE 1. Location map showing the Colorado River through Grand Canyon, with weather-station sites indicated. Six stations, active since November 2003, collect data at three sites: two stations each at 24.5 mile, Malgosa, and Palisades. One station collects data at each of three additional sites: Comanche, Forster, and 202.9 mile; these three have been in operation since April 2004. 
resources, weather stations were deployed in areas of the river corridor known to contain cultural sites but not near enough to any archaeologically significant areas that the presence of the equipment might damage these sites. All study sites have experienced a reduction in open sand area since Glen Canyon Dam was constructed (based on analysis of aerial photographs), but also experienced new deposition during the 1996 experimental flood released from the dam (Webb and others, 1999). These criteria were intended to allow monitoring of the effects of similar high-flow experiments in the event that any occurred during the twoyear duration of the weather-station program; the timing of the November 2004 flood experiment does in fact allow for its effects to be studied as part of this project. Finally, study sites were chosen away from high-visitation areas such as camping beaches, to limit access to the equipment by the many recreational visitors who use the river corridor.

\section{5 mile:}

Two instrument stations have been in operation at 24.5 mile, at the downstream end of a small debris fan on river left (the left bank of the river when facing downstream) since November 14, 2003. One of these, named Station 24.5 $\mathrm{L}$ (for "lower"), is located near the river just above the $1,270 \mathrm{~m}^{3} / \mathrm{s}\left(45,000 \mathrm{ft}^{3} / \mathrm{s}\right)$ stage at the lower end of a small aeolian dune field. The area of this dune field that lies between the two weather stations undergoes active aeolian sand transport, while an area of approximately equal size to the north (upstream) of the active dunes contains relatively inactive, deflated aeolian dunes that have a thin cover of cryptogamic crust. A moderate amount of vegetation (tamarisk shrubs and grasses) is present between this weather station and the river. The second station, $24.5 \mathrm{U}$ ("upper") is $95 \mathrm{~m}$ uphill of $24.5 \mathrm{~L}$, at the upper, northeastern end of the dune field, $\sim 20 \mathrm{~m}$ away from the cliff wall that forms the upper-elevation boundary of the dune field. 


\section{Malgosa:}

Two weather stations were set up in a very active aeolian dune field at the downstream side of the debris fan at the mouth of Malgosa Canyon (a small tributary drainage that joins the Colorado River on river right, near river mile 57.9) on November 17, 2003. Station Mal L is located near river level approximately on the $1,160 \mathrm{~m}^{3} / \mathrm{s}\left(41,000 \mathrm{ft}^{3} / \mathrm{s}\right)$ stage line, at the low-elevation, downstream (southern) end of the dune field. Minor vegetation is present between Station Mal $\mathrm{L}$ and the water line. Station Mal $U$ is located $50 \mathrm{~m}$ uphill from Mal $\mathrm{L}$ at the top (north, upstream end) of the dune field, approximately one vertical meter below the dune crest; the position of the dune crest tends to shift on time scales of weeks due to active sand transport at this site. The dune field between the two instrument stations has little vegetation and no cryptogamic soil development.

\section{Palisades:}

Two weather stations have been in operation on river left in the Palisades area (near Palisades Creek, river mile 66.1) since November 18, 2003. Station Pal $L$ is located on a relict fluvial cobble-boulder deposit immediately above the river ( $\sim 2 \mathrm{~m}$ above the $566 \mathrm{~m}^{3} / \mathrm{s}\left[20,000 \mathrm{ft}^{3} / \mathrm{s}\right]$ stage). Boulders and moderate vegetation surround this instrument site. Station $\mathrm{Pal} U$ is located $\sim 100 \mathrm{~m}$ northeast of $\mathrm{Pal} \mathrm{L}$ in a large aeolian dune field that is relatively inactive, with grasses, occasional mesquite trees, and well developed cryptogamic soil covering most dunes.

\section{Comanche:}

One weather station was deployed on river left downstream of Comanche Creek on April 20, 2004 (Station Com; river mile 68.0). This station is located in an aeolian dune field that is relatively inactive, with grass and cryptogamic-crust cover; a zone of dense vegetation dominated by mesquite trees separates this dune field from the river. 


\section{Forster:}

One weather station was deployed on river left in an active aeolian dune field at the mouth of Forster Canyon (river mile 123) on April 23, 2004. The orientation of sand shadows and dune forms in this dune field indicated that the dominant aeolian sand transport was likely to be directed up the Forster drainage, perpendicular to the trend of the river, an unusual situation that prompted further study. Sparse vegetation and occasional cryptogamic crust occur in this dune field.

\section{9 mile:}

One weather station was deployed on river right at mile 202.9 (Station 202.9) on April 28, 2004. This site includes an aeolian dune field near river level that is covered by trees and abundant arrowweed and rabbit-brush shrubs. The heavy vegetation cover has grown on the dune field and on a large river-level sand bar just downstream of the dune field in post-dam time, judging from aerial photographs, and now covers a large area of previously open sand. Immediately upstream from the instrument site, an aeolian dune field at higher elevation has experienced erosion by deflation and gully incision. Equipment installed at 202.9 mile included an anemometer and rain gauge but no sand traps. The purpose of data collection at this site is to identify the sand source for that eroding, higherelevation dune field by documenting the dominant wind direction that could cause sand transport; this will establish to what degree vegetation encroachment downstream of the site may have affected the condition of the upper dune field by limiting aeolian transport from its sand source. 


\section{METHODS}

Technical specifications of instruments used during this study are discussed below. Digital measurements of wind and rainfall are recorded on data loggers from which data are downloaded when the instrument stations are visited at regular intervals (approximately every 4-8 weeks). Mean wind velocity, maximum gust velocity, and wind direction are measured at each station with a 4-minute sampling interval and recorded as 4-minute averages. Aeolian sand flux is monitored using sand traps that are emptied once during each maintenance visit. Measurements of sand transport are therefore based on cumulative values that represent the interval between maintenance visits to the study sites.

\section{Anemometers:}

Wind velocity, maximum gust velocity, and wind direction were measured using weatherproof 'spinning cup' anemometers manufactured by Onset Computer Company of Bourne, Massachusetts ${ }^{1}$. These anemometers measure wind speed with a resolution of $0.19 \mathrm{~m} / \mathrm{s}$ (accuracy range $\pm 0.5 \mathrm{~m} / \mathrm{s}$ at wind speeds below $17 \mathrm{~m} / \mathrm{s}, \pm 3 \%$ at wind speeds 17 to $30 \mathrm{~m} / \mathrm{s}$, and $\pm 4 \%$ at wind speeds 30 to $44 \mathrm{~m} / \mathrm{s}$ ). Revolutions of the instrument's spinning cups are accumulated every three seconds for the duration of the user-defined logging interval (four minutes in this study); wind speed is then recorded as the average speed for the entire logging interval. Gust speed is recorded as the maximum three-second wind recorded during each logging interval. Wind direction, measured as vector components with a resolution of $1.4^{\circ}$ and an accuracy range of $\pm 5^{\circ}$, is accumulated every three seconds during each logging interval, and recorded as an average direction calculated from the sum of the vector components. These anemometers have a $2^{\circ}$ blind window between $358^{\circ}$ and $0^{\circ}$ in which no readings can be made.

\footnotetext{
${ }^{1}$ Use of trade, firm, or product names is for descriptive purposes only and does not imply endorsement by the U.S. Government.
} 


\section{Rain Gauges:}

Rainfall is monitored using Onset 'tipping-bucket' rain gauges. The use of two rain gauges at each of three of the study sites (24.5 mile, Malgosa, and Palisades, one at each instrument tripod) allows resolution of local precipitation events that may include precipitation that varies widely in intensity over short distances. The Onset rain gauge has a resolution of $0.2 \mathrm{~mm}$, the amount of rainfall required to trigger one 'tip' of its sensor. The accuracy to which these gauges are calibrated is $\pm 1 \%$ at rainfall rates of up to $20 \mathrm{~mm} /$ hour. Data are recorded as the number of tips per sampling interval.

\section{Data Loggers:}

Anemometers and rain gauges at each instrument station are connected to a $\mathrm{HOBO}^{\circledR}$ MicroStation weather station (data logger) manufactured by Onset. This four-channel weather station, powered by four AA batteries, contains $512 \mathrm{~kb}$ of memory. The logger is equipped with a $3.5 \mathrm{~mm}$ serial port for communication with a laptop computer via a USB port. Time accuracy is reported by Onset to be 0 to 2 seconds for the first data point, and \pm 5 seconds per week for subsequent data points when operating conditions are maintained at $\sim 25^{\circ} \mathrm{C}$. Because the logging interval used in this study is substantially longer (4 minutes) than the accuracy envelope for time, the potential drift in the logger's time accuracy is not considered significant.

\section{Sand Traps:}

A variety of sand traps have been developed for use in aeolian transport studies (for example, Goossens and others, 2000, 2001; Zobeck and others, 2003). Optimal trap design for use in aeolian studies includes the ability of traps to perform isokinetically, causing minimal distortion of air flow at the sampling orifice, and a high and well-calibrated efficiency range (Nickling and McKenna 
Neuman, 1997). The galvanized metal traps used in this study, a wedge-shaped passive-sampling design known as the Big Spring Number Eight (BSNE; Fryrear, 1986) have been shown by previous studies to perform well in both respects (Stout and Fryrear, 1989), and have the added advantage of vanes that turn the traps to face the incident wind direction. BSNE traps have a sampling orifice that is $0.05 \mathrm{~m}$ tall and $0.02 \mathrm{~m}$ wide; air flow enters the trap through this orifice and exits through a 60-mesh screen in the upper surface of the trap. Sand is retained in the lower half of the trap after falling through a wider (18-mesh) screen.

Calibration of sand trap efficiency can be a substantial source of error in field studies if not well established (McEwan and Willets, 1993; Sherman and others, 1998). The BSNE design is one of the most widely used in agricultural and sedimentological studies (Zobeck and others, 2003). Its efficiency has been calibrated by multiple wind-tunnel and field studies using a range of grain sizes for sand and dust, and over a wide range of wind velocities (Fryrear, 1986; Shao and others, 1993; Goossens and Offer, 2000; Goossens and others, 2000, 2001). Calibrations in those studies suggest an efficiency range of 70-130 percent for the range of grain sizes and velocities measured at these study sites (a truly isokinetic sand trap would have a trapping efficiency of $100 \%$; trapping efficiency will be above or below $100 \%$ if the sand-trap design directs air flow into the trap or away from it, respectively).

\section{Field Deployment:}

Three of the weather stations were initially deployed with three anemometers arranged at different heights on an instrument tripod, to allow resolution of a vertical wind-velocity profile: $24.5 \mathrm{U}$, Mal L, and Pal U. These three stations operated with three anemometers between November 2003 and April 2004. In April 2004, one anemometer was removed from $24.5 \mathrm{U}$ and one from $\mathrm{Pal} U$; these two were redeployed with equipment set up at three new sites (Comanche, Forster, 202.9 mile) at that time. Mal L has been using only one anemometer since May 2004. Station Mal U was deployed with two anemometers but since May 2004 has used three. Stations 24.5 L, Mal L, Pal L, 
and Com were deployed with two anemometers each; $24.5 \mathrm{~L}$ and $\mathrm{Pal} \mathrm{L}$ continue to use two anemometers each. The station at Forster (For) was initially deployed in April 2004 with two anemometers, but has been operated with only one since May 2004, when it was discovered that frequent high winds and very active sand transport at that site caused substantial damage to the anemometer deployed closer to ground level. The station at 202.9 mile has been operated with only one anemometer throughout its use (since April 2004).

For stations at which three anemometers were deployed, they are mounted on the tripod mast at heights of 2.0,1.0, and $0.5 \mathrm{~m}$ and connected to a data logger. For stations that include only two anemometers, the heights are set at 2.0 and $0.5 \mathrm{~m}$ (with the exception of Station $\mathrm{Pal} \mathrm{L}$, where the lower anemometer is at $1.0 \mathrm{~m}$ to reduce interference with boulders). Using a compass, the directional reading of each anemometer is re-calibrated at each maintenance visit. Instrument tripods are equipped with a copper-plated grounding rod and copper grounding wire clamped to the metal tripod, to reduce instrument damage in the event of a lightning strike.

An array of BSNE sand traps is set up $\sim 3$ to $5 \mathrm{~m}$ from each instrument tripod (with the exception of the 202.9 mile station, at which no sand traps are used) to collect wind-blown sand. Four BSNE traps are mounted on a vertical pole; these traps are equipped with vanes to turn them into the wind. Sand trap heights are set at 1.0, 0.7, 0.4, and $0.1 \mathrm{~m}$. Station Pal L uses only three traps, because very little sand transport occurs given its location in a boulder field; heights of sand traps at Pal $L$ are $1.0,0.5$, and $0.25 \mathrm{~m}$.

To minimize visual impacts to canyon visitors, instruments were made as inconspicuous as possible using paint and vegetation camouflage (fig. 2). At each site, vegetation along the river level provides some screening so that instruments are not immediately obvious from river level. In the event that instruments are noticed by river runners or hikers, the tripods are labeled with signs that briefly explain their function and the purpose of the research to educate visitors and minimize the chance of equipment disruption. 


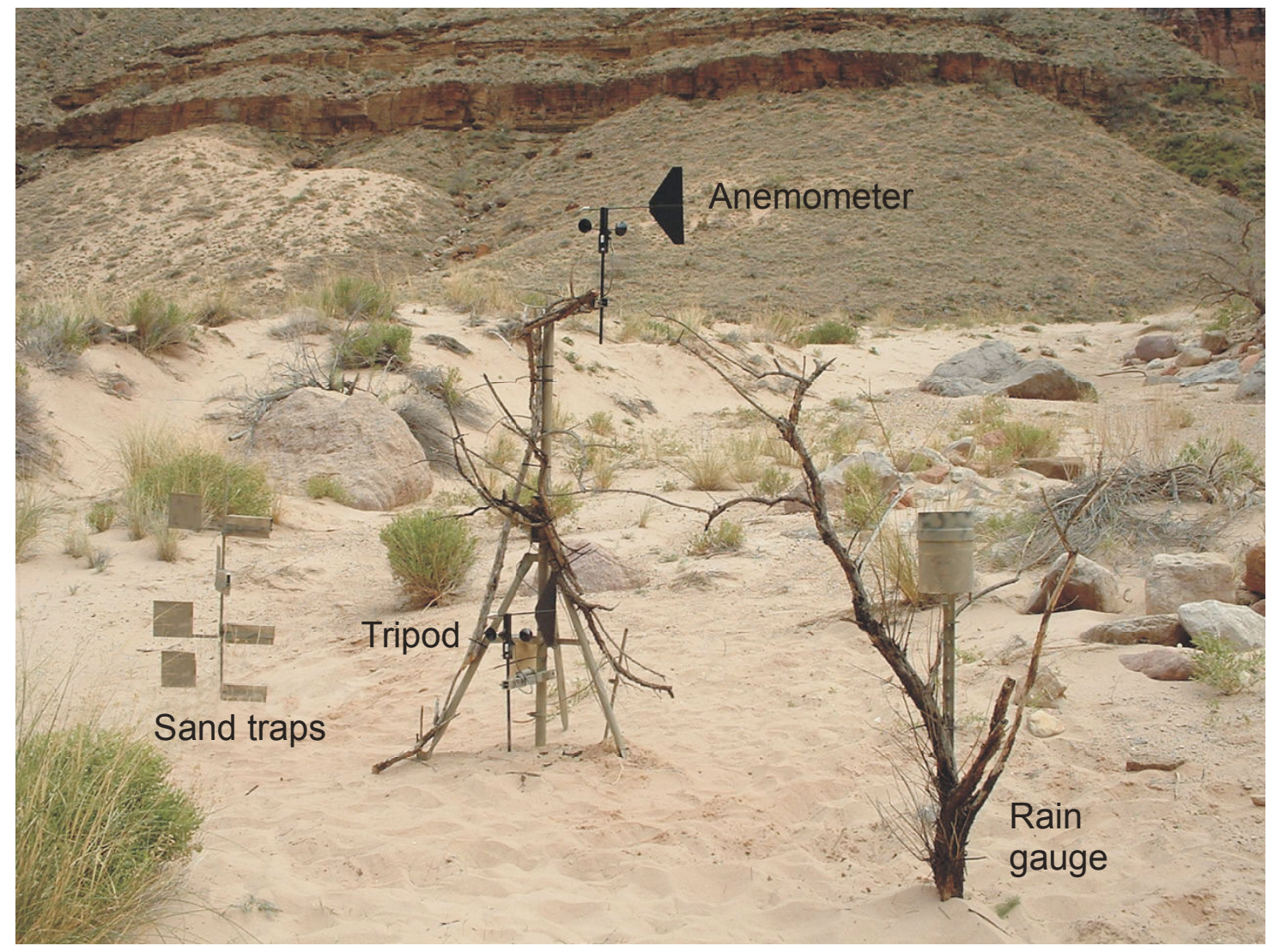

FIGURE 2. Photograph of one instrument station, deployed at the mouth of Forster Canyon. Four sand traps are set up on a vertical pole at the left side of the photo. A tripod with two anemometers (at heights $2.0 \mathrm{~m}$ and 0.5 $m$ above ground level) is in the center; the data logger is attached to this tripod also but is not visible. On the right side of the photograph is a tipping-bucket rain gauge. Equipment has been spray-painted and camouflaged with dead branches to reduce visibility. 


\section{Sediment Collection and Analysis:}

The total sand mass collected from each trap during each data download is weighed. Mass-transport rates are calculated for each interval between downloads by integration from 0 to $1 \mathrm{~m}$ of a curve fit to the mass-vs.-height data. Because the great majority of aeolian sediment transport takes place near the bed, the amount of sediment transport represented by the lowermost $1 \mathrm{~m}$ will generally account for over 99 percent of the actual transport that occurred during each time interval (Zobeck and Fryrear, 1986; Vories and Fryrear, 1991; Sterk and Raats, 1996; Zobeck and others, 2003).

The curve-fitting procedure uses a five-parameter combined power-law and exponential function, as this has been shown to model vertical aeolian mass flux more accurately than either power-law (Zobeck and Fryrear, 1986; Fryrear and others, 1991) or exponential (Vories and Fryrear, 1991) fits alone (Sterk and Raats, 1996). The actual mass flux is considered to span an efficiency range of $70-130 \%$ for the BSNE traps with the grain size at the various study locations and the wind speeds measured (Goossens and others, 2000).

Grain-size analyses were completed for representative samples of sand at the location of each instrument tripod at the time of initial deployment. Sediment samples were analyzed using a Beckman Coulter LS 100Q laser particle-size analyzer linked to a LS variable-speed fluid module. This instrument uses 126 detector rings to measure the pattern of diffracted light intensities as a function of the angle from the axis of the original laser beam to compute the particle-size distribution. Median grain size $\left(d_{50}\right)$ for these 'grab samples' from each of the study sites is shown in Table 1. These particle-size data allowed estimation of the critical threshold of motion at these sites, that is, the wind velocity needed to mobilize and transport sand grains of a certain size. Using the formulation developed by Bagnold (1941), the critical threshold of motion for sand of the grain sizes found at these study sites is approximately $2 \mathrm{~m} / \mathrm{s}$. 


\begin{tabular}{|c|c|}
\hline \multicolumn{2}{|c|}{$\mathrm{d}_{50 \text { (microns) }}$} \\
\hline 24.5 mile $L$ & 198.8 \\
\hline 24.5 mile U & 214.6 \\
\hline Mal L & 204 \\
\hline Mal U & 312.9 \\
\hline Pal L & boulders \\
\hline Pal U & 156.7 \\
\hline Com & N/A \\
\hline For & 225.7 \\
\hline 202.5 mile & 210.3 \\
\hline
\end{tabular}

Table 1. Median grain size, $d_{50}$, for sand at the locations of the nine weather stations, measured by Coulter laser particle-size analysis. Station Pal $L$ is located in a cobble/boulder bar; the others are on sand deposits. 


\section{Data Processing:}

Data downloaded from the data loggers (wind speed, gust speed, wind direction, and rainfall) were exported from Onset Boxcar ${ }^{\mathrm{TM}}$ software into Microsoft Excel spreadsheets and then into Mathworks ${ }^{\text {TM MATLAB software for }}$ subsequent manipulation and analysis. The first four and last four data points of each data set are deleted to remove the influence of maintenance activities on the recorded data. These deleted data points represent 16 minutes after the data logger was launched at the completion of the previous download, and 16 minutes prior to downloading the present data set.

In analyzing wind data collected during this study, it is useful to consider not only wind velocity but the potential for aeolian sediment transport that can result from a given wind velocity. We use a proxy variable to represent the potential for sediment transport due to wind velocity. This variable, $Q p$, is calculated for data points in which wind velocity $(u)$ exceeds the critical threshold of motion $\left(u_{c r i t}\right)$, and is defined as the difference between the measured wind velocity and the critical threshold of motion (taken to be $2 \mathrm{~m} / \mathrm{s}$ ), raised to the third power:

$$
Q p=\left(u \square u_{c r i t}\right)^{3}
$$

This relationship between wind velocity and potential for sediment transport follows the convention used to develop aeolian sediment-transport models such as those of Kawamura (1951) and Lettau and Lettau (1977), but substitutes wind velocity for shear velocity $(u *)$. The variable $Q p$ has units of $\mathrm{m}^{3} \mathrm{~s}^{-3}$; while the units do not directly translate to a mass flux, comparing spatial and temporal variations in the relative magnitude of $Q p$ values yields information about the potential for sediment redistribution by wind. For data points in which wind velocity is less than $u_{\text {crit, }} Q p$ is set equal to zero (no sediment transport can occur). 


\section{RESULTS}

Files containing complete weather records from these instrument stations in Microsoft Excel $\square$ format are available to be downloaded at http://pubs.usgs.gov/of/2005/1309/. An example of a high-resolution wind-velocity record for one month (average wind velocity, collected every four minutes) is shown in figure 3. This record is from May, 2004, at Station Mal $U$, and represents conditions during the season with the highest wind velocities and greatest recorded sediment-transport rates at this site. At this resolution, it is apparent that wind conditions vary diurnally, with highest velocity occurring typically in the afternoons. Wind direction often varies on a diurnal cycle also (fig. 3 ), with the direction in this record coming from the south (directed upstream) most consistently in the afternoon. Given that sediment transport is expected to occur only when wind speed exceeds $\sim 2 \mathrm{~m} / \mathrm{s}$, the greatest potential for transport in the record shown in figure 3 would also be directed upstream (wind blowing from the south).

Diurnally averaged wind conditions are reported for each individual station and discussed in more detail below. Vector sums indicating the magnitude and direction of potential sediment transport $(Q p)$ are listed by month for each weather station in Table 2.

Precipitation data for November 2003 through December 2004 are summarized in figure 4 and in tables 3 and 4 . Total rain amounts vary substantially with location throughout the river corridor (tab. 3), and the same event may induce precipitation with great spatial variability (tab. 4). It is common for daily rainfall amounts at the upper and lower rain gauge at a single location to differ by several tens of millimeters (for example, records from 1/31/04 or 2/3/04 at 24.5 mile and Malgosa; tab. 4). In other cases, the two rain gauges at one location may record daily rainfall totals that differ by several $\mathrm{mm}$ (for example, on 4/8/04 at 24.5 mile, where rainfall was $5.2 \mathrm{~mm}$ and $2.6 \mathrm{~mm}$ at Stations $24.5 \mathrm{~L}$ and $24.5 \mathrm{U}$, respectively; tab. 4). Occasionally, strong but very isolated summer storms were recorded, such as an event on 6/29/04 that brought $15.0 \mathrm{~mm}$ and 


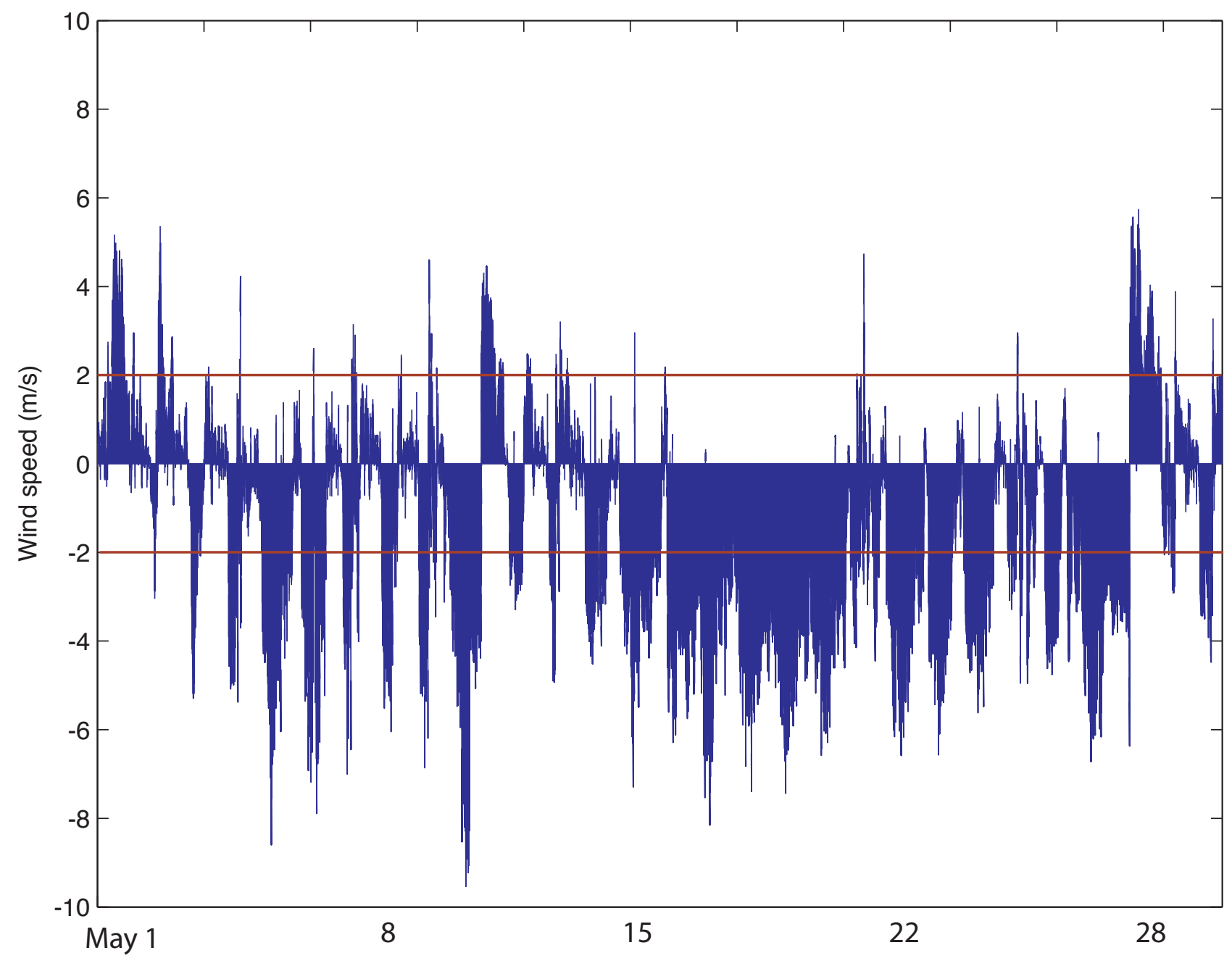

Figure 3. Example of high-resolution wind-speed data for one month at one weather station; data were collected during May 2004 at the upper of the two stations at Malgosa, river mile 57.9 (Station Mal U). Data represent wind speed averaged over four-minute intervals, sampled every four minutes. Line lengths represent magnitude of wind speed for each data point; orientation of the lines indicates the direction from which the wind blew. At Malgosa, the trend of the main canyon is approximately north-south, such that the dominant wind directions (upstream and downstream) are readily apparent. Wind speeds tend to increase during the afternoons and to assume a more consistent direction compared with other times of day. The critical threshold of motion for sand of grain size $\sim 300$ microns (at this site the median grain size, $d_{50}$, is 313 microns) is approximately $2 \mathrm{~m} / \mathrm{s}$; wind velocities capable of transporting sand must exceed this (red horizontal lines). For this month, the direction of dominant transport at this site would be from the south, directed upstream. 


\begin{tabular}{|c|c|c|c|c|c|c|c|c|c|c|c|c|c|c|}
\hline Station & Nov-03 & Dec-03 & Jan-04 & Feb-04 & Mar-04 & Apr-04 & May-04 & Jun-04 & Jul-04 & Aug-04 & Sep-04 & Oct-04 & Nov-04 & Dec-04 \\
\hline $24.5 \mathrm{~L}$ & 1361,273 & 485,248 & 142,163 & 189,260 & 345,237 & 1667,242 & 1203,230 & 1250,244 & 192,150 & 555,254 & 1048,246 & 1283,269 & 49,118 & 7,230 \\
\hline $24.5 \mathrm{U}$ & 4240,243 & 1542,225 & 135,155 & 874,248 & 1218,229 & 7330,244 & 7382,234 & 6157,211 & 2520,205 & 3747,217 & 4118,233 & 2960,254 & 399,220 & 36,226 \\
\hline Mal L & 4120,129 & 9349,131 & 2869,139 & 6226,140 & 2376,155 & 21771,135 & 35033,122 & 15468,147 & 2761,181 & 2693,136 & 9997,124 & 7187,126 & 73,308 & 3569,113 \\
\hline Mal U & 17734,153 & 29082,149 & 1555,197 & 50752,161 & 18531,158 & 110100,163 & 160030,138 & 104300,144 & 59612,134 & 48205,136 & 102490,134 & 99518,135 & 3711,115 & 14531,142 \\
\hline Pal L & 1113,164 & 8254,145 & 6755,149 & 2197,171 & 439,319 & 13393,159 & 8633,142 & 2771,217 & 1291,352 & 2061,231 & 2668,116 & 2436,124 & 850,13 & 5626,106 \\
\hline Pal U & 10869,150 & 34311,139 & 22740,144 & 9451,138 & 4184,107 & 107440,165 & 144340,159 & 32301,149 & 19968,185 & 33732,185 & 52451,146 & 39481,141 & 8553,90 & 20177,80 \\
\hline Com & & & & & & 1528,102 & 5136,140 & 1030,53 & 2394,15 & 1144,87 & 1589,149 & 1008,127 & 1465,6 & 719,57 \\
\hline For & & & & & & 55385,29 & 65151,18 & 29764,15 & 14945,22 & 11320,24 & 28060,19 & 2828,31 & 8740,20 & 3545,17 \\
\hline 202.9 & & & & & & 334,31 & 1675,196 & 841,166 & 461,300 & 223,302 & 588,139 & 275,235 & & \\
\hline
\end{tabular}

Table 2a

\begin{tabular}{|c|c|c|c|c|c|c|c|c|c|c|c|c|c|c|}
\hline Station & Nov-03 & Dec-03 & Jan-04 & Feb-04 & Mar-04 & Apr-04 & May-04 & Jun-04 & Jul-04 & Aug-04 & Sep-04 & Oct-04 & Nov-04 & Dec-04 \\
\hline $24.5 \mathrm{~L}$ & 1361,273 & 106,266 & 59,149 & 158,258 & 325,243 & 1893,236 & 1203,230 & 1313,244 & 265,121 & 346,272 & 994.249 & 1284.268 & 41,113 & 4.47 \\
\hline $24.5 \mathrm{U}$ & 4240,243 & 477,232 & 55,163 & 784,236 & 1252,227 & 6972,245 & 7382,234 & 6194,212 & 1817,200 & 1887,219 & 3478,234 & 2857,255 & 398,220 & 13,197 \\
\hline Mal L & 4120,129 & 5699,130 & 1823,141 & 1780,154 & 1803,156 & 18112,134 & 35033,122 & 1548,147 & 2982,178 & 1700,128 & 4159,116 & 4465,121 & 76,313 & 1339,117 \\
\hline Mal U & 17734,153 & 23225,152 & 2667,155 & 12712,158 & 12244,154 & 96329,163 & 160030,138 & 104220,144 & 59028,135 & 24797,135 & 55496,135 & 43462,133 & 5946,141 & 7247,140 \\
\hline Pal L & 1113,164 & 2117,128 & 6519,149 & 1283,320 & 610,239 & 13528,159 & 8547,142 & 2540,219 & 1546,352 & 1747,200 & 1536,109 & 1996,120 & 780,47 & 4850,327 \\
\hline Pal U & 10869,150 & 11306,104 & 20057,145 & 2437,97 & 3211,111 & 109640,166 & 144340,159 & 34924,152 & 17737,185 & 30925,190 & 28094,145 & 31084,138 & 8395,103 & 30201,5 \\
\hline Com & & & & & & 1528,102 & 5136,140 & 849,70 & 2299,15 & 893,123 & 1318,151 & 794,120 & 1192,9 & 1193,1 \\
\hline For & & & & & & 55385,29 & 65151,18 & 29709,15 & 14860,22 & 5356,21 & 19314,20 & 2759,29 & 8713,20 & 35,359 \\
\hline 202.9 & & & & & & 334,31 & 1675,196 & 841,166 & 76,238 & 26,302 & & & & \\
\hline
\end{tabular}

Table 2b 
Table 2. Vector sums of the sediment-transport proxy variable, $Q p$, by month for each weather station. Vector sums were calculated using wind velocity and direction measurements from the 2.0-m anemometer at each station, and are reported as the magnitude of $Q p\left(\right.$ in $\left.m^{3} s^{-3}\right)$ followed by the direction from which this net transport would occur, in degrees. a) vector sums for all months at all stations irrespective of rain events. b) vector sums re-calculated using wind data only from time when sand is assumed to have been dry enough to transport. Values in bold text are those that differ from the corresponding values in part a. These calculations (in part b) omit wind data collected within 48 hours of a rainfall event. Records at 24.5 mile, Malgosa, and Palisades begin on November 14, 17, and 18, 2003, respectively. Records for Comanche, Forster, and 202.9 mile begin on April 20,23, and 28, 2004, respectively. The record at Mal $L$ does not include data between November 17 and December 9, 2004, when that station was disabled during the flood experiment. The record from Mal $\mathrm{U}$ is missing $\sim 10$ hours of data from February and March 2004. The record at Forster is missing data between October 21 and November 23, 2004. The record from 202.9 mile is missing data from September 11 through September 23, from November 6 through November 24, and for all days after November 26, 2004. 




Figure 4. Average monthly rainfall in the Colorado River corridor in Grand Canyon. Values are calculated for November 2003 through April 2004 from the monthly rainfall from weather stations at 24.5 mile, Malgosa, and Palisades (using one mean value for each of these sites, each of which has two instrument stations). For the period from May through December, 2004, values are calculated as the mean of the total for each of the six instrumented locations, excluding data from 202.9 mile between September and December 2004 when the rain gauge at that site was not functioning. 
$14.0 \mathrm{~mm}$ of rain to Stations $24.5 \mathrm{~L}$ and $24.5 \mathrm{U}$, respectively, while no other rain gauges recorded any precipitation that day (tab. 4). The highest precipitation rates during this study interval were measured in the late summer and fall, when monsoon storms tend to dominate rainfall (fig. 4; for example, Hereford and others, 2002). The single event that resulted in the greatest total rainfall during this study interval occurred on the night of September 18-19, 2004, when a dissipating Pacific cyclonic storm (named Javier) brought rain that varied between $\sim 20$ and $\sim 50 \mathrm{~mm}$ locally (with $52.4 \mathrm{~mm}$ recorded at Station Mal L that night).

\section{5 mile:}

The record at Station $24.5 \mathrm{~L}$ begins at 1814 hours on November 14, 2003, and continues through December 31, 2004 with no interruptions other than routine maintenance. The record at Station $24.5 \mathrm{U}$ begins at 1823 hours on November 14, 2003, and continues through December 31, 2004 with no interruptions other than routine maintenance. Sand-transport data, diurnally averaged wind velocity, diurnal measurements of maximum gust speed, and total daily rainfall for Station $24.5 \mathrm{~L}$ are shown in figure 5 . Sediment-transport potential by direction for January through December 2004 is summarized in the rose diagram in figure 6. A vector sum of the data for calendar year 2004 at this station yields a net $Q p$ magnitude of 7,350 $\mathrm{m}^{3} \mathrm{~s}^{-3}$ from a direction of 244 degrees.

Sand-transport, wind, and precipitation data for Station $24.5 \mathrm{U}$ are shown in figure 7; potential sediment transport there is summarized in figure 8 (for comparison purposes, figs. $6 a$ and $8 a$ are plotted on the same scale). A vector sum of the data for calendar year 2004 at Station $24.5 U$ yields a net $Q p$ magnitude of $35,800 \mathrm{~m}^{3} \mathrm{~s}^{-3}$ from a direction of 229 degrees. Rates of sediment transport measured at Station $24.5 \mathrm{U}$ were greater than those measured at Station $24.5 \mathrm{~L}$ by a factor of two to three during most of the year; this agrees with higher wind velocities measured at Station $24.5 \mathrm{U}$, which resulted in greater potential for sediment transport (fig. 8). Net aeolian sediment transport is thus directed upstream with respect to the river, oriented such that sand will be 


\begin{tabular}{c|ccccccccc} 
& $24.5 \mathrm{~L}$ & $24.5 \mathrm{U}$ & Mal L & Mal U & Pal L & Pal U & Com & For & 202.9 \\
\hline Nov-03 & 0 & 0 & 0 & 0 & 0 & 0 & & & \\
Dec-03 & 1.4 & 1.4 & 3.4 & 3.4 & 5.2 & 4.8 & & & \\
Jan-04 & 1.4 & 1.6 & 16.2 & 17.0 & 11.6 & 10.4 & & & \\
Feb-04 & 6.6 & 6.8 & 9.6 & 9.6 & 3.8 & 3.2 & & & \\
Mar-04 & 3.0 & 3.0 & 1.8 & 1.6 & 9.0 & 10.0 & & & \\
Apr-04 & 15.0 & 14.0 & 8.2 & 7.8 & 11.4 & 11.2 & 0 & 0 & 0 \\
May-04 & 0 & 0 & 0 & 0 & 0.4 & 0 & 0 & 0 & 0 \\
Jun-04 & 15.4 & 14.2 & 0.4 & 0.4 & 2.8 & 2.2 & 1.2 & 0.4 & 0 \\
Jul-04 & 3.0 & 2.8 & 16.2 & 18.8 & 3.4 & 3.4 & 3.8 & 9.2 & 24.0 \\
Aug-04 & 28.4 & 25.2 & 60.4 & 64.4 & 45.0 & 45.2 & 43.0 & 43.0 & 35.6 \\
Sep-04 & 57.0 & 55.8 & 65.0 & 64.8 & 34.2 & 31.8 & 37.4 & 40.0 & \\
Oct-04 & 11.6 & 11.8 & 9.2 & 9.8 & 6.0 & 5.6 & 7.0 & 41.4 & \\
Nov-04 & 61.8 & 60.6 & & 67.0 & 45.6 & 32.4 & 51.4 & 4.0 & \\
Dec-04 & 20.2 & 19.8 & & 32.6 & 17.2 & 16.6 & 19.0 & 17.4 &
\end{tabular}

Table 3. Total rainfall, in $\mathrm{mm}$, received each month at each of the weather stations. Records at 24.5 mile, Malgosa, and Palisades begin on November 14, 17, and 18, 2003, respectively. Records for Comanche, Forster, and 202.9 mile begin on April 20, 23, and 28, 2004, respectively. The record for Mal $L$ in November and December 2004 is not reported because the station had been disabled during the November flood experiment. Records for 202.9 mile for September through December are not reported because that station required repairs to the rain gauge and experienced malfunction of the data logger during that time. 
Table 4. Total rainfall, in $\mathrm{mm}$, received daily at each of the weather stations.

Records at 24.5 mile, Malgosa, and Palisades begin on November 14, 17, and 18, 2003, respectively. Records for Comanche, Forster, and 202.9 mile begin on April 20, 23, and 28, 2004, respectively. The record for Mal $L$ in November and December 2004 is not reported because the station had been disabled during the November flood experiment. Records for 202.9 mile for September through December are not reported because that station required repairs to the rain gauge and experienced malfunction of the data logger during that time.

\begin{tabular}{|c|c|c|c|c|c|c|c|c|c|c|}
\hline & $24.5 \mathrm{~L}$ & $24.5 \mathrm{U}$ & Mal L & Mal U & Pal L & Pal U & Com & For & 202.9 & Year Day \\
\hline $11 / 14 / 03$ & 0 & 0 & N/A & $\mathrm{N} / \mathrm{A}$ & $\mathrm{N} / \mathrm{A}$ & $N / A$ & $N / A$ & $\mathrm{~N} / \mathrm{A}$ & $\mathrm{N} / \mathrm{A}$ & 318 \\
\hline $11 / 15 / 03$ & 0 & 0 & N/A & $\mathrm{N} / \mathrm{A}$ & $\mathrm{N} / \mathrm{A}$ & $\mathrm{N} / \mathrm{A}$ & $\mathrm{N} / \mathrm{A}$ & $\mathrm{N} / \mathrm{A}$ & $\mathrm{N} / \mathrm{A}$ & 319 \\
\hline $11 / 16 / 03$ & 0 & 0 & N/A & $\mathrm{N} / \mathrm{A}$ & $\mathrm{N} / \mathrm{A}$ & $\mathrm{N} / \mathrm{A}$ & $\mathrm{N} / \mathrm{A}$ & $\mathrm{N} / \mathrm{A}$ & $\mathrm{N} / \mathrm{A}$ & 320 \\
\hline $11 / 17 / 03$ & 0 & 0 & 0 & 0 & $\mathrm{~N} / \mathrm{A}$ & $N / A$ & $\mathrm{~N} / \mathrm{A}$ & $\mathrm{N} / \mathrm{A}$ & $\mathrm{N} / \mathrm{A}$ & 321 \\
\hline $11 / 18 / 03$ & 0 & 0 & 0 & 0 & 0 & 0 & $\mathrm{~N} / \mathrm{A}$ & $\mathrm{N} / \mathrm{A}$ & $\mathrm{N} / \mathrm{A}$ & 322 \\
\hline $11 / 19 / 03$ & 0 & 0 & 0 & 0 & 0 & 0 & $\mathrm{~N} / \mathrm{A}$ & $\mathrm{N} / \mathrm{A}$ & $\mathrm{N} / \mathrm{A}$ & 323 \\
\hline $11 / 20 / 03$ & 0 & 0 & 0 & 0 & 0 & 0 & N/A & $\mathrm{N} / \mathrm{A}$ & N/A & 324 \\
\hline $11 / 21 / 03$ & 0 & 0 & 0 & 0 & 0 & 0 & $\mathrm{~N} / \mathrm{A}$ & $\mathrm{N} / \mathrm{A}$ & $\mathrm{N} / \mathrm{A}$ & 325 \\
\hline $11 / 22 / 03$ & 0 & 0 & 0 & 0 & 0 & 0 & $\mathrm{~N} / \mathrm{A}$ & $\mathrm{N} / \mathrm{A}$ & $\mathrm{N} / \mathrm{A}$ & 326 \\
\hline $11 / 23 / 03$ & 0 & 0 & 0 & 0 & 0 & 0 & $\mathrm{~N} / \mathrm{A}$ & $\mathrm{N} / \mathrm{A}$ & $\mathrm{N} / \mathrm{A}$ & 327 \\
\hline $11 / 24 / 03$ & 0 & 0 & 0 & 0 & 0 & 0 & $\mathrm{~N} / \mathrm{A}$ & $\mathrm{N} / \mathrm{A}$ & $\mathrm{N} / \mathrm{A}$ & 328 \\
\hline $11 / 25 / 03$ & 0 & 0 & 0 & 0 & 0 & 0 & $\mathrm{~N} / \mathrm{A}$ & $\mathrm{N} / \mathrm{A}$ & $\mathrm{N} / \mathrm{A}$ & 329 \\
\hline $11 / 26 / 03$ & 0 & 0 & 0 & 0 & 0 & 0 & $\mathrm{~N} / \mathrm{A}$ & $\mathrm{N} / \mathrm{A}$ & $\mathrm{N} / \mathrm{A}$ & 330 \\
\hline $11 / 27 / 03$ & 0 & 0 & 0 & 0 & 0 & 0 & N/A & $\mathrm{N} / \mathrm{A}$ & N/A & 331 \\
\hline $11 / 28 / 03$ & 0 & 0 & 0 & 0 & 0 & 0 & $\mathrm{~N} / \mathrm{A}$ & $\mathrm{N} / \mathrm{A}$ & $\mathrm{N} / \mathrm{A}$ & 332 \\
\hline $11 / 29 / 03$ & 0 & 0 & 0 & 0 & 0 & 0 & $\mathrm{~N} / \mathrm{A}$ & $\mathrm{N} / \mathrm{A}$ & N/A & 333 \\
\hline $11 / 30 / 03$ & 0 & 0 & 0 & 0 & 0 & 0 & $\mathrm{~N} / \mathrm{A}$ & $\mathrm{N} / \mathrm{A}$ & $\mathrm{N} / \mathrm{A}$ & 334 \\
\hline $12 / 1 / 03$ & 0 & 0 & 0 & 0 & 0 & 0 & $N / A$ & $\mathrm{~N} / \mathrm{A}$ & $\mathrm{N} / \mathrm{A}$ & 335 \\
\hline $12 / 2 / 03$ & 0 & 0 & 0 & 0 & 0 & 0 & $\mathrm{~N} / \mathrm{A}$ & $\mathrm{N} / \mathrm{A}$ & N/A & 336 \\
\hline $12 / 3 / 03$ & 0 & 0 & 0 & 0 & 0 & 0 & $\mathrm{~N} / \mathrm{A}$ & $\mathrm{N} / \mathrm{A}$ & $\mathrm{N} / \mathrm{A}$ & 337 \\
\hline $12 / 4 / 03$ & 0 & 0 & 0 & 0 & 0 & 0 & $\mathrm{~N} / \mathrm{A}$ & $\mathrm{N} / \mathrm{A}$ & $\mathrm{N} / \mathrm{A}$ & 338 \\
\hline $12 / 5 / 03$ & 0 & 0 & 0 & 0 & 0 & 0 & $\mathrm{~N} / \mathrm{A}$ & $\mathrm{N} / \mathrm{A}$ & $\mathrm{N} / \mathrm{A}$ & 339 \\
\hline $12 / 6 / 03$ & 0 & 0 & 0 & 0 & 0 & 0 & $\mathrm{~N} / \mathrm{A}$ & $\mathrm{N} / \mathrm{A}$ & $\mathrm{N} / \mathrm{A}$ & 340 \\
\hline 12/7/03 & 0 & 0 & 0 & 0.2 & 0 & 0 & $\mathrm{~N} / \mathrm{A}$ & $\mathrm{N} / \mathrm{A}$ & $\mathrm{N} / \mathrm{A}$ & 341 \\
\hline $12 / 8 / 03$ & 0.4 & 0.4 & 0.6 & 0.6 & 1.0 & 0.8 & $\mathrm{~N} / \mathrm{A}$ & $\mathrm{N} / \mathrm{A}$ & $\mathrm{N} / \mathrm{A}$ & 342 \\
\hline $12 / 9 / 03$ & 0 & 0 & 0 & 0 & 0 & 0 & $\mathrm{~N} / \mathrm{A}$ & $\mathrm{N} / \mathrm{A}$ & N/A & 343 \\
\hline $12 / 10 / 03$ & 0 & 0 & 0 & 0 & 0 & 0 & $\mathrm{~N} / \mathrm{A}$ & $\mathrm{N} / \mathrm{A}$ & $\mathrm{N} / \mathrm{A}$ & 344 \\
\hline $12 / 11 / 03$ & 0 & 0 & 0.2 & 0.2 & 1.4 & 1.2 & $\mathrm{~N} / \mathrm{A}$ & $\mathrm{N} / \mathrm{A}$ & $\mathrm{N} / \mathrm{A}$ & 345 \\
\hline $12 / 12 / 03$ & 0 & 0 & 0 & 0 & 0 & 0 & $\mathrm{~N} / \mathrm{A}$ & $\mathrm{N} / \mathrm{A}$ & $\mathrm{N} / \mathrm{A}$ & 346 \\
\hline $12 / 13 / 03$ & 0 & 0 & 0 & 0 & 0 & 0 & $\mathrm{~N} / \mathrm{A}$ & $\mathrm{N} / \mathrm{A}$ & N/A & 347 \\
\hline
\end{tabular}




\begin{tabular}{|c|c|c|c|c|c|c|c|c|c|c|}
\hline $12 / 14 / 03$ & 0.6 & 0.6 & 1.0 & 1.2 & 1.4 & 1.4 & $\mathrm{~N} / \mathrm{A}$ & N/A & N/A & 348 \\
\hline $12 / 15 / 03$ & 0 & 0 & 0 & 0 & 0 & 0 & N/A & N/A & N/A & 349 \\
\hline $12 / 16 / 03$ & 0 & 0 & 0 & 0 & 0 & 0 & N/A & N/A & N/A & 350 \\
\hline $12 / 17 / 03$ & 0 & 0 & 0 & 0 & 0 & 0 & N/A & N/A & $N / A$ & 351 \\
\hline $12 / 18 / 03$ & 0 & 0 & 0 & 0 & 0 & 0 & N/A & N/A & N/A & 352 \\
\hline $12 / 19 / 03$ & 0 & 0 & 0 & 0 & 0 & 0 & N/A & N/A & N/A & 353 \\
\hline $12 / 20 / 03$ & 0 & 0 & 0 & 0 & 0 & 0 & N/A & N/A & N/A & 354 \\
\hline $12 / 21 / 03$ & 0 & 0 & 0 & 0 & 0 & 0 & N/A & N/A & N/A & 355 \\
\hline $12 / 22 / 03$ & 0 & 0 & 0 & 0 & 0 & 0 & N/A & N/A & $\mathrm{N} / \mathrm{A}$ & 356 \\
\hline $12 / 23 / 03$ & 0 & 0 & 0 & 0 & 0 & 0 & N/A & N/A & N/A & 357 \\
\hline $12 / 24 / 03$ & 0 & 0 & 0 & 0 & 0 & 0 & N/A & N/A & N/A & 358 \\
\hline $12 / 25 / 03$ & 0 & 0 & 0 & 0 & 0 & 0 & N/A & N/A & N/A & 359 \\
\hline $12 / 26 / 03$ & 0.4 & 0.4 & 1.4 & 1.2 & 1.4 & 1.4 & N/A & N/A & N/A & 360 \\
\hline $12 / 27 / 03$ & 0 & 0 & 0 & 0 & 0 & 0 & N/A & N/A & $\mathrm{N} / \mathrm{A}$ & 361 \\
\hline $12 / 28 / 03$ & 0 & 0 & 0 & 0 & 0 & 0 & N/A & N/A & N/A & 362 \\
\hline $12 / 29 / 03$ & 0 & 0 & 0 & 0 & 0 & 0 & N/A & N/A & $N / A$ & 363 \\
\hline $12 / 30 / 03$ & 0 & 0 & 0 & 0 & 0 & 0 & N/A & N/A & N/A & 364 \\
\hline $12 / 31 / 03$ & 0 & 0 & 0 & 0 & 0 & 0 & N/A & N/A & N/A & 365 \\
\hline $1 / 1 / 04$ & 0 & 0 & 0 & 0 & 0 & 0 & N/A & N/A & N/A & 1 \\
\hline $1 / 2 / 04$ & 0 & 0 & 1.4 & 1.6 & 0.4 & 0.4 & N/A & N/A & N/A & 2 \\
\hline $1 / 3 / 04$ & 0.2 & 0.2 & 10.8 & 11.0 & 4.6 & 4.2 & N/A & N/A & N/A & 3 \\
\hline $1 / 4 / 04$ & 0 & 0 & 0 & 0 & 0 & 0 & N/A & N/A & N/A & 4 \\
\hline $1 / 5 / 04$ & 0 & 0 & 0 & 0 & 0 & 0 & N/A & N/A & N/A & 5 \\
\hline $1 / 6 / 04$ & 0 & 0 & 0 & 0 & 0 & 0 & N/A & N/A & N/A & 6 \\
\hline $1 / 7 / 04$ & 0 & 0 & 0 & 0 & 0 & 0 & N/A & N/A & N/A & 7 \\
\hline $1 / 8 / 04$ & 0 & 0 & 0 & 0 & 0 & 0 & N/A & N/A & N/A & 8 \\
\hline $1 / 9 / 04$ & 0 & 0 & 0 & 0 & 0 & 0 & N/A & N/A & N/A & 9 \\
\hline $1 / 10 / 04$ & 0 & 0 & 0 & 0 & 0 & 0 & N/A & N/A & N/A & 10 \\
\hline $1 / 11 / 04$ & 0 & 0 & 0 & 0 & 0 & 0 & N/A & N/A & N/A & 11 \\
\hline $1 / 12 / 04$ & 0 & 0 & 0 & 0 & 0 & 0 & N/A & N/A & N/A & 12 \\
\hline $1 / 13 / 04$ & 0 & 0 & 0 & 0 & 0 & 0 & N/A & N/A & N/A & 13 \\
\hline $1 / 14 / 04$ & 0 & 0 & 0 & 0 & 0 & 0 & N/A & N/A & N/A & 14 \\
\hline $1 / 15 / 04$ & 0 & 0 & 0 & 0 & 0 & 0 & N/A & N/A & N/A & 15 \\
\hline $1 / 16 / 04$ & 0 & 0 & 0 & 0 & 0 & 0 & N/A & N/A & N/A & 16 \\
\hline $1 / 17 / 04$ & 0 & 0 & 0 & 0 & 0 & 0 & N/A & N/A & $N / A$ & 17 \\
\hline $1 / 18 / 04$ & 0 & 0 & 0 & 0 & 0 & 0 & N/A & N/A & N/A & 18 \\
\hline $1 / 19 / 04$ & 0 & 0 & 0.8 & 0.6 & 0.2 & 0.2 & N/A & N/A & N/A & 19 \\
\hline $1 / 20 / 04$ & 0.2 & 0.2 & 1.0 & 1.2 & 4.2 & 3.8 & N/A & N/A & N/A & 20 \\
\hline $1 / 21 / 04$ & 0 & 0 & 0 & 0 & 0 & 0 & N/A & N/A & N/A & 21 \\
\hline $1 / 22 / 04$ & 0 & 0 & 0 & 0 & 0 & 0 & N/A & N/A & N/A & 22 \\
\hline $1 / 23 / 04$ & 0 & 0 & 0 & 0 & 0 & 0 & N/A & N/A & N/A & 23 \\
\hline $1 / 24 / 04$ & 0 & 0 & 0 & 0 & 0 & 0 & N/A & N/A & N/A & 24 \\
\hline $1 / 25 / 04$ & 0 & 0 & 0 & 0 & 0.4 & 0 & N/A & N/A & N/A & 25 \\
\hline
\end{tabular}




\begin{tabular}{|c|c|c|c|c|c|c|c|c|c|}
\hline $1 / 26 / 04$ & 0 & 0 & 0 & 0 & 0 & 0 & N/A & $\mathrm{N} / \mathrm{A}$ & N/A \\
\hline $1 / 27 / 04$ & 0 & 0 & 0 & 0 & 0 & 0 & N/A & $\mathrm{N} / \mathrm{A}$ & $\mathrm{N} / \mathrm{A}$ \\
\hline $1 / 28 / 04$ & 0 & 0 & 0 & 0 & 0 & 0 & N/A & $\mathrm{N} / \mathrm{A}$ & N/A \\
\hline $1 / 29 / 04$ & 0 & 0 & 0 & 0 & 0 & 0 & N/A & $\mathrm{N} / \mathrm{A}$ & N/A \\
\hline $1 / 30 / 04$ & 0 & 0 & 0 & 0 & 0 & 0 & N/A & $\mathrm{N} / \mathrm{A}$ & N/A \\
\hline $1 / 31 / 04$ & 1.0 & 1.2 & 2.2 & 2.6 & 1.8 & 1.8 & N/A & $\mathrm{N} / \mathrm{A}$ & N/A \\
\hline $2 / 1 / 04$ & 0 & 0 & 0 & 0 & 0 & 0 & N/A & $\mathrm{N} / \mathrm{A}$ & N/A \\
\hline $2 / 2 / 04$ & 0 & 0 & 0 & 0 & 0 & 0 & N/A & $\mathrm{N} / \mathrm{A}$ & N/A \\
\hline $2 / 3 / 04$ & 1.2 & 1.4 & 2.6 & 2.4 & 0.8 & 0.8 & N/A & $\mathrm{N} / \mathrm{A}$ & N/A \\
\hline $2 / 4 / 04$ & 0.2 & 0 & 0.2 & 0.2 & 0 & 0 & N/A & $\mathrm{N} / \mathrm{A}$ & N/A \\
\hline $2 / 5 / 04$ & 0 & 0 & 0 & 0 & 0 & 0 & N/A & $\mathrm{N} / \mathrm{A}$ & N/A \\
\hline $2 / 6 / 04$ & 0 & 0 & 0 & 0 & 0 & 0 & $\mathrm{~N} / \mathrm{A}$ & $\mathrm{N} / \mathrm{A}$ & N/A \\
\hline $2 / 7 / 04$ & 0 & 0 & 0 & 0 & 0 & 0 & N/A & $\mathrm{N} / \mathrm{A}$ & N/A \\
\hline $2 / 8 / 04$ & 0 & 0 & 0 & 0 & 0 & 0 & N/A & $\mathrm{N} / \mathrm{A}$ & N/A \\
\hline $2 / 9 / 04$ & 0 & 0 & 0 & 0 & 0 & 0 & N/A & $\mathrm{N} / \mathrm{A}$ & N/A \\
\hline 2/10/04 & 0 & 0 & 0 & 0 & 0 & 0 & N/A & $\mathrm{N} / \mathrm{A}$ & $\mathrm{N} / \mathrm{A}$ \\
\hline $2 / 11 / 04$ & 0 & 0 & 0 & 0 & 0 & 0 & $\mathrm{~N} / \mathrm{A}$ & $\mathrm{N} / \mathrm{A}$ & N/A \\
\hline $2 / 12 / 04$ & 0 & 0 & 0 & 0 & 0 & 0 & N/A & $\mathrm{N} / \mathrm{A}$ & N/A \\
\hline 2/13/04 & 0 & 0 & 0 & 0 & 0 & 0 & $\mathrm{~N} / \mathrm{A}$ & $\mathrm{N} / \mathrm{A}$ & N/A \\
\hline $2 / 14 / 04$ & 0 & 0 & 0 & 0 & 0 & 0 & N/A & $\mathrm{N} / \mathrm{A}$ & N/A \\
\hline 2/15/04 & 0 & 0 & 0 & 0 & 0 & 0 & N/A & $\mathrm{N} / \mathrm{A}$ & N/A \\
\hline 2/16/04 & 0 & 0 & 0 & 0 & 0 & 0 & N/A & $\mathrm{N} / \mathrm{A}$ & N/A \\
\hline 2/17/04 & 0 & 0 & 0 & 0 & 0 & 0 & $\mathrm{~N} / \mathrm{A}$ & $\mathrm{N} / \mathrm{A}$ & N/A \\
\hline 2/18/04 & 0 & 0 & 0 & 0 & 0 & 0 & $\mathrm{~N} / \mathrm{A}$ & $\mathrm{N} / \mathrm{A}$ & N/A \\
\hline 2/19/04 & 0 & 0 & 0 & 0 & 0 & 0 & N/A & $\mathrm{N} / \mathrm{A}$ & N/A \\
\hline $2 / 20 / 04$ & 0 & 0 & 0 & 0 & 0 & 0 & N/A & $\mathrm{N} / \mathrm{A}$ & $\mathrm{N} / \mathrm{A}$ \\
\hline $2 / 21 / 04$ & 1.6 & 1.4 & 1.4 & 1.4 & 0.6 & 0.4 & N/A & $\mathrm{N} / \mathrm{A}$ & $\mathrm{N} / \mathrm{A}$ \\
\hline $2 / 22 / 04$ & 0 & 0.2 & 0.6 & 0.6 & 0.2 & 0.4 & N/A & $\mathrm{N} / \mathrm{A}$ & N/A \\
\hline $2 / 23 / 04$ & 2.8 & 3.0 & 2.2 & 2.2 & 1.0 & 0.8 & N/A & $\mathrm{N} / \mathrm{A}$ & N/A \\
\hline $2 / 24 / 04$ & 0 & 0 & 0 & 0 & 0.2 & 0 & $\mathrm{~N} / \mathrm{A}$ & $\mathrm{N} / \mathrm{A}$ & N/A \\
\hline $2 / 25 / 04$ & 0 & 0 & 0 & 0 & 0 & 0 & $\mathrm{~N} / \mathrm{A}$ & $\mathrm{N} / \mathrm{A}$ & N/A \\
\hline $2 / 26 / 04$ & 0 & 0 & 0.4 & 0.4 & 0.4 & 0.2 & N/A & $\mathrm{N} / \mathrm{A}$ & N/A \\
\hline $2 / 27 / 04$ & 0.8 & 0.8 & 2.2 & 2.4 & 0.6 & 0.6 & $\mathrm{~N} / \mathrm{A}$ & $\mathrm{N} / \mathrm{A}$ & $\mathrm{N} / \mathrm{A}$ \\
\hline $2 / 28 / 04$ & 0 & 0 & 0 & 0 & 0 & 0 & N/A & $\mathrm{N} / \mathrm{A}$ & $\mathrm{N} / \mathrm{A}$ \\
\hline 2/29/04 & 0 & 0 & 0 & 0 & 0 & 0 & N/A & $\mathrm{N} / \mathrm{A}$ & N/A \\
\hline $3 / 1 / 04$ & 0 & 0 & 0 & 0 & 0 & 0 & N/A & $\mathrm{N} / \mathrm{A}$ & N/A \\
\hline $3 / 2 / 04$ & 0 & 0 & 1.4 & 1.2 & 4.6 & 5.4 & $\mathrm{~N} / \mathrm{A}$ & $\mathrm{N} / \mathrm{A}$ & N/A \\
\hline $3 / 3 / 04$ & 0 & 0 & 0 & 0 & 0 & 0 & N/A & $\mathrm{N} / \mathrm{A}$ & N/A \\
\hline $3 / 4 / 04$ & 0 & 0 & 0 & 0 & 0 & 0 & N/A & $\mathrm{N} / \mathrm{A}$ & N/A \\
\hline $3 / 5 / 04$ & 0 & 0 & 0 & 0 & 0 & 0 & N/A & $\mathrm{N} / \mathrm{A}$ & $\mathrm{N} / \mathrm{A}$ \\
\hline $3 / 6 / 04$ & 0 & 0 & 0 & 0 & 0 & 0 & N/A & $\mathrm{N} / \mathrm{A}$ & $\mathrm{N} / \mathrm{A}$ \\
\hline $3 / 7 / 04$ & 0 & 0 & 0 & 0 & 0 & 0 & N/A & $\mathrm{N} / \mathrm{A}$ & N/A \\
\hline $3 / 8 / 04$ & 0 & 0 & 0 & 0 & 0 & 0 & N/A & $\mathrm{N} / \mathrm{A}$ & N/A \\
\hline
\end{tabular}




\begin{tabular}{|c|c|c|c|c|c|c|c|c|c|c|}
\hline $3 / 9 / 04$ & 0 & 0 & 0 & 0 & 0 & 0 & $\mathrm{~N} / \mathrm{A}$ & N/A & N/A & 69 \\
\hline 3/10/04 & 0 & 0 & 0 & 0 & 0 & 0 & $\mathrm{~N} / \mathrm{A}$ & $\mathrm{N} / \mathrm{A}$ & $\mathrm{N} / \mathrm{A}$ & 70 \\
\hline $3 / 11 / 04$ & 0 & 0 & 0 & 0 & 0 & 0 & $\mathrm{~N} / \mathrm{A}$ & $\mathrm{N} / \mathrm{A}$ & N/A & 71 \\
\hline $3 / 12 / 04$ & 2.6 & 2.6 & 2.6 & 0 & 0 & 0 & N/A & N/A & N/A & 72 \\
\hline $3 / 13 / 04$ & 0 & 0 & 0 & 0 & 0 & 0 & N/A & N/A & $\mathrm{N} / \mathrm{A}$ & 73 \\
\hline $3 / 14 / 04$ & 0 & 0 & 0 & 0 & 0 & 0 & N/A & N/A & $\mathrm{N} / \mathrm{A}$ & 74 \\
\hline $3 / 15 / 04$ & 0 & 0 & 0 & 0 & 0 & 0 & $\mathrm{~N} / \mathrm{A}$ & N/A & N/A & 75 \\
\hline $3 / 16 / 04$ & 0 & 0 & 0 & 0 & 0 & 0 & $\mathrm{~N} / \mathrm{A}$ & $\mathrm{N} / \mathrm{A}$ & N/A & 76 \\
\hline $3 / 17 / 04$ & 0 & 0 & 0 & 0 & 0 & 0 & $\mathrm{~N} / \mathrm{A}$ & N/A & N/A & 77 \\
\hline $3 / 18 / 04$ & 0 & 0 & 0 & 0 & 0 & 0 & $\mathrm{~N} / \mathrm{A}$ & $\mathrm{N} / \mathrm{A}$ & N/A & 78 \\
\hline $3 / 19 / 04$ & 0 & 0 & 0 & 0 & 0 & 0 & $\mathrm{~N} / \mathrm{A}$ & N/A & $\mathrm{N} / \mathrm{A}$ & 79 \\
\hline $3 / 20 / 04$ & 0 & 0 & 0 & 0 & 0 & 0 & $\mathrm{~N} / \mathrm{A}$ & $\mathrm{N} / \mathrm{A}$ & $\mathrm{N} / \mathrm{A}$ & 80 \\
\hline $3 / 21 / 04$ & 0 & 0 & 0 & 0 & 0 & 0 & $\mathrm{~N} / \mathrm{A}$ & N/A & $\mathrm{N} / \mathrm{A}$ & 81 \\
\hline $3 / 22 / 04$ & 0 & 0 & 0 & 0 & 0 & 0 & $\mathrm{~N} / \mathrm{A}$ & N/A & N/A & 82 \\
\hline $3 / 23 / 04$ & 0 & 0 & 0.4 & 0.4 & 4.2 & 4.6 & $\mathrm{~N} / \mathrm{A}$ & $\mathrm{N} / \mathrm{A}$ & N/A & 83 \\
\hline $3 / 24 / 04$ & 0 & 0 & 0 & 0 & 0 & 0 & $\mathrm{~N} / \mathrm{A}$ & $\mathrm{N} / \mathrm{A}$ & N/A & 84 \\
\hline $3 / 25 / 04$ & 0 & 0 & 0 & 0 & 0 & 0 & $\mathrm{~N} / \mathrm{A}$ & $\mathrm{N} / \mathrm{A}$ & $\mathrm{N} / \mathrm{A}$ & 85 \\
\hline $3 / 26 / 04$ & 0.2 & 0.2 & 0.2 & 0 & 0 & 0 & N/A & N/A & $\mathrm{N} / \mathrm{A}$ & 86 \\
\hline $3 / 27 / 04$ & 0 & 0 & 0 & 0 & 0 & 0 & $\mathrm{~N} / \mathrm{A}$ & $\mathrm{N} / \mathrm{A}$ & $\mathrm{N} / \mathrm{A}$ & 87 \\
\hline $3 / 28 / 04$ & 0 & 0 & 0 & 0 & 0 & 0 & $\mathrm{~N} / \mathrm{A}$ & N/A & N/A & 88 \\
\hline $3 / 29 / 04$ & 0 & 0 & 0 & 0 & 0 & 0 & $\mathrm{~N} / \mathrm{A}$ & N/A & N/A & 89 \\
\hline $3 / 30 / 04$ & 0 & 0 & 0 & 0 & 0 & 0 & $\mathrm{~N} / \mathrm{A}$ & N/A & N/A & 90 \\
\hline $3 / 31 / 04$ & 0 & 0 & 0 & 0 & 0.2 & 0.2 & $\mathrm{~N} / \mathrm{A}$ & $\mathrm{N} / \mathrm{A}$ & $\mathrm{N} / \mathrm{A}$ & 91 \\
\hline $4 / 1 / 04$ & 0 & 0 & 0 & 0 & 0 & 0 & $\mathrm{~N} / \mathrm{A}$ & $\mathrm{N} / \mathrm{A}$ & $\mathrm{N} / \mathrm{A}$ & 92 \\
\hline $4 / 2 / 04$ & 2.2 & 2.2 & 4.2 & 4.4 & 5.6 & 5.6 & N/A & N/A & $\mathrm{N} / \mathrm{A}$ & 93 \\
\hline $4 / 3 / 04$ & 3.8 & 3.8 & 2.2 & 2.0 & 2.8 & 2.8 & $\mathrm{~N} / \mathrm{A}$ & $\mathrm{N} / \mathrm{A}$ & N/A & 94 \\
\hline $4 / 4 / 04$ & 3.6 & 3.8 & 1.2 & 1.2 & 0 & 0 & $\mathrm{~N} / \mathrm{A}$ & N/A & N/A & 95 \\
\hline $4 / 5 / 04$ & 0.2 & 0 & 0.2 & 0.2 & 0.2 & 0.2 & $\mathrm{~N} / \mathrm{A}$ & $\mathrm{N} / \mathrm{A}$ & N/A & 96 \\
\hline $4 / 6 / 04$ & 0 & 0 & 0 & 0 & 0 & 0 & $\mathrm{~N} / \mathrm{A}$ & $\mathrm{N} / \mathrm{A}$ & $\mathrm{N} / \mathrm{A}$ & 97 \\
\hline $4 / 7 / 04$ & 0 & 0 & 0 & 0 & 0.6 & 0.6 & $\mathrm{~N} / \mathrm{A}$ & $\mathrm{N} / \mathrm{A}$ & $\mathrm{N} / \mathrm{A}$ & 98 \\
\hline $4 / 8 / 04$ & 5.2 & 2.6 & 2.6 & 0 & 0 & 0 & $\mathrm{~N} / \mathrm{A}$ & $\mathrm{N} / \mathrm{A}$ & $\mathrm{N} / \mathrm{A}$ & 99 \\
\hline $4 / 9 / 04$ & 0 & 0 & 0 & 0 & 0 & 0 & $\mathrm{~N} / \mathrm{A}$ & N/A & N/A & 100 \\
\hline $4 / 10 / 04$ & 0 & 0 & 0.2 & 0.2 & 2.0 & 2.0 & $\mathrm{~N} / \mathrm{A}$ & $\mathrm{N} / \mathrm{A}$ & $\mathrm{N} / \mathrm{A}$ & 101 \\
\hline $4 / 11 / 04$ & 0 & 0 & 0 & 0 & 0 & 0 & $\mathrm{~N} / \mathrm{A}$ & N/A & N/A & 102 \\
\hline $4 / 12 / 04$ & 0 & 0 & 0 & 0 & 0 & 0 & $\mathrm{~N} / \mathrm{A}$ & N/A & N/A & 103 \\
\hline $4 / 13 / 04$ & 0 & 0 & 0 & 0 & 0 & 0 & $\mathrm{~N} / \mathrm{A}$ & $\mathrm{N} / \mathrm{A}$ & $\mathrm{N} / \mathrm{A}$ & 104 \\
\hline $4 / 14 / 04$ & 0 & 0 & 0 & 0 & 0 & 0 & $\mathrm{~N} / \mathrm{A}$ & $\mathrm{N} / \mathrm{A}$ & $\mathrm{N} / \mathrm{A}$ & 105 \\
\hline $4 / 15 / 04$ & 0 & 0 & 0 & 0 & 0 & 0 & N/A & N/A & N/A & 106 \\
\hline $4 / 16 / 04$ & 0 & 0 & 0 & 0 & 0 & 0 & $\mathrm{~N} / \mathrm{A}$ & $\mathrm{N} / \mathrm{A}$ & N/A & 107 \\
\hline $4 / 17 / 04$ & 0 & 0 & 0 & 0 & 0 & 0 & $\mathrm{~N} / \mathrm{A}$ & $\mathrm{N} / \mathrm{A}$ & $\mathrm{N} / \mathrm{A}$ & 108 \\
\hline $4 / 18 / 04$ & 0 & 0 & 0.2 & 0.2 & 0.2 & 0 & $\mathrm{~N} / \mathrm{A}$ & $\mathrm{N} / \mathrm{A}$ & N/A & 109 \\
\hline $4 / 19 / 04$ & 0 & 0 & 0 & 0 & 0 & 0 & $\mathrm{~N} / \mathrm{A}$ & $\mathrm{N} / \mathrm{A}$ & N/A & 110 \\
\hline $4 / 20 / 04$ & 0 & 0 & 0 & 0 & 0 & 0 & 0 & $\mathrm{~N} / \mathrm{A}$ & $\mathrm{N} / \mathrm{A}$ & 111 \\
\hline
\end{tabular}




\begin{tabular}{|c|c|c|c|c|c|c|c|c|c|c|}
\hline $4 / 21 / 04$ & 0 & 0 & 0 & 0 & 0 & 0 & 0 & $\mathrm{~N} / \mathrm{A}$ & N/A & 112 \\
\hline $4 / 22 / 04$ & 0 & 0 & 0 & 0 & 0 & 0 & 0 & $\mathrm{~N} / \mathrm{A}$ & $\mathrm{N} / \mathrm{A}$ & 113 \\
\hline $4 / 23 / 04$ & 0 & 0 & 0 & 0 & 0 & 0 & 0 & 0 & N/A & 114 \\
\hline $4 / 24 / 04$ & 0 & 0 & 0 & 0 & 0 & 0 & 0 & 0 & N/A & 115 \\
\hline $4 / 25 / 04$ & 0 & 0 & 0 & 0 & 0 & 0 & 0 & 0 & N/A & 116 \\
\hline $4 / 26 / 04$ & 0 & 0 & 0 & 0 & 0 & 0 & 0 & 0 & N/A & 117 \\
\hline $4 / 27 / 04$ & 0 & 0 & 0 & 0 & 0 & 0 & 0 & 0 & N/A & 118 \\
\hline $4 / 28 / 04$ & 0 & 0 & 0 & 0 & 0 & 0 & 0 & 0 & 0 & 119 \\
\hline $4 / 29 / 04$ & 0 & 0 & 0 & 0 & 0 & 0 & 0 & 0 & 0 & 120 \\
\hline $4 / 30 / 04$ & 0 & 0 & 0 & 0 & 0 & 0 & 0 & 0 & 0 & 121 \\
\hline $5 / 1 / 04$ & 0 & 0 & 0 & 0 & 0 & 0 & 0 & 0 & 0 & 122 \\
\hline $5 / 2 / 04$ & 0 & 0 & 0 & 0 & 0 & 0 & 0 & 0 & 0 & 123 \\
\hline $5 / 3 / 04$ & 0 & 0 & 0 & 0 & 0 & 0 & 0 & 0 & 0 & 124 \\
\hline $5 / 4 / 04$ & 0 & 0 & 0 & 0 & 0 & 0 & 0 & 0 & 0 & 125 \\
\hline $5 / 5 / 04$ & 0 & 0 & 0 & 0 & 0 & 0 & 0 & 0 & 0 & 126 \\
\hline $5 / 6 / 04$ & 0 & 0 & 0 & 0 & 0 & 0 & 0 & 0 & 0 & 127 \\
\hline $5 / 7 / 04$ & 0 & 0 & 0 & 0 & 0 & 0 & 0 & 0 & 0 & 128 \\
\hline $5 / 8 / 04$ & 0 & 0 & 0 & 0 & 0 & 0 & 0 & 0 & 0 & 129 \\
\hline $5 / 9 / 04$ & 0 & 0 & 0 & 0 & 0 & 0 & 0 & 0 & 0 & 130 \\
\hline $5 / 10 / 04$ & 0 & 0 & 0 & 0 & 0 & 0 & 0 & 0 & 0 & 131 \\
\hline $5 / 11 / 04$ & 0 & 0 & 0 & 0 & 0 & 0 & 0 & 0 & 0 & 132 \\
\hline $5 / 12 / 04$ & 0 & 0 & 0 & 0 & 0 & 0 & 0 & 0 & 0 & 133 \\
\hline $5 / 13 / 04$ & 0 & 0 & 0 & 0 & 0 & 0 & 0 & 0 & 0 & 134 \\
\hline $5 / 14 / 04$ & 0 & 0 & 0 & 0 & 0 & 0 & 0 & 0 & 0 & 135 \\
\hline $5 / 15 / 04$ & 0 & 0 & 0 & 0 & 0 & 0 & 0 & 0 & 0 & 136 \\
\hline $5 / 16 / 04$ & 0 & 0 & 0 & 0 & 0 & 0 & 0 & 0 & 0 & 137 \\
\hline $5 / 17 / 04$ & 0 & 0 & 0 & 0 & 0 & 0 & 0 & 0 & 0 & 138 \\
\hline $5 / 18 / 04$ & 0 & 0 & 0 & 0 & 0 & 0 & 0 & 0 & 0 & 139 \\
\hline $5 / 19 / 04$ & 0 & 0 & 0 & 0 & 0 & 0 & 0 & 0 & 0 & 140 \\
\hline $5 / 20 / 04$ & 0 & 0 & 0 & 0 & 0.4 & 0 & 0 & 0 & 0 & 141 \\
\hline $5 / 21 / 04$ & 0 & 0 & 0 & 0 & 0 & 0 & 0 & 0 & 0 & 142 \\
\hline $5 / 22 / 04$ & 0 & 0 & 0 & 0 & 0 & 0 & 0 & 0 & 0 & 143 \\
\hline $5 / 23 / 04$ & 0 & 0 & 0 & 0 & 0 & 0 & 0 & 0 & 0 & 144 \\
\hline $5 / 24 / 04$ & 0 & 0 & 0 & 0 & 0 & 0 & 0 & 0 & 0 & 145 \\
\hline $5 / 25 / 04$ & 0 & 0 & 0 & 0 & 0 & 0 & 0 & 0 & 0 & 146 \\
\hline $5 / 26 / 04$ & 0 & 0 & 0 & 0 & 0 & 0 & 0 & 0 & 0 & 147 \\
\hline $5 / 27 / 04$ & 0 & 0 & 0 & 0 & 0 & 0 & 0 & 0 & 0 & 148 \\
\hline $5 / 28 / 04$ & 0 & 0 & 0 & 0 & 0 & 0 & 0 & 0 & 0 & 149 \\
\hline $5 / 29 / 04$ & 0 & 0 & 0 & 0 & 0 & 0 & 0 & 0 & 0 & 150 \\
\hline $5 / 30 / 04$ & 0 & 0 & 0 & 0 & 0 & 0 & 0 & 0 & 0 & 151 \\
\hline $5 / 31 / 04$ & 0 & 0 & 0 & 0 & 0 & 0 & 0 & 0 & 0 & 152 \\
\hline $6 / 1 / 04$ & 0 & 0 & 0 & 0 & 0 & 0 & 0 & 0 & 0 & 153 \\
\hline $6 / 2 / 04$ & 0 & 0 & 0 & 0 & 0 & 0 & 0 & 0 & 0 & 154 \\
\hline
\end{tabular}




\begin{tabular}{|c|c|c|c|c|c|c|c|c|c|c|}
\hline $6 / 3 / 04$ & 0 & 0 & 0 & 0 & 0 & 0 & 0 & 0 & 0 & 155 \\
\hline $6 / 4 / 04$ & 0 & 0 & 0 & 0 & 0 & 0 & 0 & 0 & 0 & 156 \\
\hline 6/5/04 & 0 & 0 & 0 & 0 & 0 & 0 & 0 & 0 & 0 & 157 \\
\hline 6/6/04 & 0 & 0 & 0 & 0 & 0 & 0 & 0 & 0 & 0 & 158 \\
\hline 6/7/04 & 0 & 0 & 0 & 0 & 0 & 0 & 0 & 0 & 0 & 159 \\
\hline 6/8/04 & 0 & 0 & 0 & 0 & 0 & 0 & 0 & 0 & 0 & 160 \\
\hline 6/9/04 & 0 & 0 & 0 & 0 & 0.2 & 0 & 0 & 0 & 0 & 161 \\
\hline $6 / 10 / 04$ & 0 & 0 & 0 & 0 & 0 & 0 & 0 & 0 & 0 & 162 \\
\hline $6 / 11 / 04$ & 0 & 0 & 0 & 0 & 0 & 0 & 0 & 0 & 0 & 163 \\
\hline $6 / 12 / 04$ & 0 & 0 & 0 & 0 & 0 & 0 & 0 & 0 & 0 & 164 \\
\hline $6 / 13 / 04$ & 0 & 0 & 0 & 0 & 0 & 0 & 0 & 0 & 0 & 165 \\
\hline $6 / 14 / 04$ & 0 & 0 & 0 & 0 & 0 & 0 & 0 & 0 & 0 & 166 \\
\hline $6 / 15 / 04$ & 0 & 0 & 0 & 0 & 0 & 0 & 0 & 0 & 0 & 167 \\
\hline $6 / 16 / 04$ & 0 & 0 & 0 & 0 & 0 & 0 & 0 & 0 & 0 & 168 \\
\hline $6 / 17 / 04$ & 0 & 0 & 0 & 0 & 0 & 0 & 0 & 0 & 0 & 169 \\
\hline $6 / 18 / 04$ & 0 & 0 & 0 & 0 & 0 & 0 & 0 & 0 & 0 & 170 \\
\hline $6 / 19 / 04$ & 0 & 0 & 0 & 0 & 0 & 0 & 0 & 0 & 0 & 171 \\
\hline $6 / 20 / 04$ & 0 & 0 & 0 & 0 & 0 & 0 & 0 & 0 & 0 & 172 \\
\hline $6 / 21 / 04$ & 0.4 & 0.2 & 0 & 0 & 0 & 0 & 0 & 0 & 0 & 173 \\
\hline $6 / 22 / 04$ & 0 & 0 & 0 & 0 & 0 & 0 & 0 & 0 & 0 & 174 \\
\hline $6 / 23 / 04$ & 0 & 0 & 0 & 0 & 0 & 0 & 0 & 0 & 0 & 175 \\
\hline $6 / 24 / 04$ & 0 & 0 & 0 & 0 & 0 & 0 & 0 & 0 & 0 & 176 \\
\hline $6 / 25 / 04$ & 0 & 0 & 0 & 0 & 0.8 & 0.8 & 0.2 & 0.4 & 0 & 177 \\
\hline $6 / 26 / 04$ & 0 & 0 & 0 & 0 & 0 & 0 & 0 & 0 & 0 & 178 \\
\hline $6 / 27 / 04$ & 0 & 0 & 0 & 0 & 0 & 0 & 0.2 & 0 & 0 & 179 \\
\hline $6 / 28 / 04$ & 0 & 0 & 0.4 & 0.4 & 1.8 & 1.4 & 0.8 & 0 & 0 & 180 \\
\hline $6 / 29 / 04$ & 15.0 & 14.0 & 0 & 0 & 0 & 0 & 0 & 0 & 0 & 181 \\
\hline $6 / 30 / 04$ & 0 & 0 & 0 & 0 & 0 & 0 & 0 & 0 & 0 & 182 \\
\hline $7 / 1 / 04$ & 0 & 0 & 0 & 0 & 0 & 0 & 0 & 0 & 0 & 183 \\
\hline $7 / 2 / 04$ & 0 & 0 & 0 & 0 & 0 & 0 & 0 & 0 & 0 & 184 \\
\hline $7 / 3 / 04$ & 0 & 0 & 0 & 0 & 0 & 0 & 0 & 0 & 0 & 185 \\
\hline $7 / 4 / 04$ & 0 & 0 & 0 & 0 & 0 & 0 & 0 & 0 & 0 & 186 \\
\hline $7 / 5 / 04$ & 0 & 0 & 0 & 0 & 0 & 0 & 0 & 0 & 0 & 187 \\
\hline $7 / 6 / 04$ & 0 & 0 & 0 & 0 & 0 & 0 & 0 & 0 & 0 & 188 \\
\hline $7 / 7 / 04$ & 0 & 0 & 0 & 0 & 0 & 0 & 0 & 0 & 0 & 189 \\
\hline $7 / 8 / 04$ & 0 & 0 & 0 & 0 & 0 & 0 & 0 & 0 & 0 & 190 \\
\hline $7 / 9 / 04$ & 0 & 0 & 0 & 0 & 0 & 0 & 0 & 0 & 0 & 191 \\
\hline $7 / 10 / 04$ & 0 & 0 & 0 & 0 & 0 & 0 & 0 & 0 & 0 & 192 \\
\hline $7 / 11 / 04$ & 0 & 0 & 0 & 0 & 0 & 0 & 0 & 0 & 0 & 193 \\
\hline $7 / 12 / 04$ & 0 & 0 & 0 & 0 & 0 & 0 & 0 & 0 & 0 & 194 \\
\hline $7 / 13 / 04$ & 0 & 0 & 0 & 0 & 0 & 0 & 0 & 0 & 0 & 195 \\
\hline $7 / 14 / 04$ & 0 & 0 & 0 & 0 & 0 & 0 & 0.4 & 0 & 0 & 196 \\
\hline $7 / 15 / 04$ & 0 & 0 & 0 & 0 & 0 & 0 & 0 & 0 & 0 & 197 \\
\hline
\end{tabular}




\begin{tabular}{|c|c|c|c|c|c|c|c|c|c|c|}
\hline $7 / 16 / 04$ & 0 & 0 & 0 & 0 & 0.6 & 0.6 & 0 & 0 & 0.4 & 198 \\
\hline $7 / 17 / 04$ & 0 & 0 & 0 & 0 & 0 & 0 & 0 & 0 & 0 & 199 \\
\hline $7 / 18 / 04$ & 2.6 & 2.6 & 0 & 0 & 0 & 0 & 0 & 0 & 0.8 & 200 \\
\hline $7 / 19 / 04$ & 0 & 0 & 3.2 & 4.6 & 0 & 0 & 0 & 0 & 0.2 & 201 \\
\hline $7 / 20 / 04$ & 0 & 0 & 2.6 & 2.8 & 0 & 0 & 0 & 0 & 0 & 202 \\
\hline $7 / 21 / 04$ & 0 & 0 & 0.4 & 0.4 & 0 & 0 & 0 & 0 & 0 & 203 \\
\hline $7 / 22 / 04$ & 0 & 0 & 0 & 0 & 0 & 0 & 0 & 0 & 0 & 204 \\
\hline $7 / 23 / 04$ & 0 & 0 & 0.4 & 0.4 & 0 & 0 & 0 & 0 & 0 & 205 \\
\hline $7 / 24 / 04$ & 0 & 0 & 0 & 0 & 0 & 0 & 0 & 0 & 0 & 206 \\
\hline $7 / 25 / 04$ & 0 & 0 & 0 & 0 & 0 & 0 & 0 & 0 & 0 & 207 \\
\hline $7 / 26 / 04$ & 0 & 0 & 0.4 & 0.6 & 0 & 0 & 0 & 2.2 & 22.2 & 208 \\
\hline $7 / 27 / 04$ & 0.2 & 0 & 9.2 & 10.0 & 2.8 & 2.8 & 3.4 & 7.0 & 0.4 & 209 \\
\hline $7 / 28 / 04$ & 0.2 & 0.2 & 0 & 0 & 0 & 0 & 0 & 0 & 0 & 210 \\
\hline $7 / 29 / 04$ & 0 & 0 & 0 & 0 & 0 & 0 & 0 & 0 & 0 & 211 \\
\hline $7 / 30 / 04$ & 0 & 0 & 0 & 0 & 0 & 0 & 0 & 0 & 0 & 212 \\
\hline $7 / 31 / 04$ & 0 & 0 & 0 & 0 & 0 & 0 & 0 & 0 & 0 & 213 \\
\hline $8 / 1 / 04$ & 0 & 0 & 0 & 0 & 1.4 & 1.4 & 0.6 & 0 & 0.8 & 214 \\
\hline $8 / 2 / 04$ & 0.4 & 0.2 & 1.6 & 2.0 & 0 & 0 & 0 & 0 & 0 & 215 \\
\hline $8 / 3 / 04$ & 0 & 0 & 0 & 0 & 0 & 0 & 0 & 0 & 0 & 216 \\
\hline $8 / 4 / 04$ & 3.0 & 3.0 & 2.8 & 3.0 & 2.0 & 2.4 & 1.8 & 1.8 & 0 & 217 \\
\hline $8 / 5 / 04$ & 0.2 & 0.2 & 5.4 & 5.6 & 1.8 & 1.8 & 3.8 & 13.2 & 0 & 218 \\
\hline $8 / 6 / 04$ & 7.0 & 6.4 & 3.0 & 3.2 & 6.6 & 6.6 & 14.4 & 19.4 & 0 & 219 \\
\hline $8 / 7 / 04$ & 0 & 0 & 0 & 0 & 0 & 0 & 0 & 0 & 0 & 220 \\
\hline $8 / 8 / 04$ & 0 & 0 & 0 & 0 & 0 & 0 & 0 & 0 & 0 & 221 \\
\hline $8 / 9 / 04$ & 0 & 0 & 0 & 0 & 0 & 0 & 0 & 0 & 0 & 222 \\
\hline 8/10/04 & 0 & 0 & 0.2 & 0.2 & 0 & 0 & 0 & 0 & 0 & 223 \\
\hline $8 / 11 / 04$ & 0 & 0 & 0 & 0 & 0 & 0 & 0 & 0 & 0 & 224 \\
\hline $8 / 12 / 04$ & 0 & 0 & 0 & 0 & 0 & 0 & 0.4 & 0 & 1.4 & 225 \\
\hline $8 / 13 / 04$ & 0 & 0 & 0 & 0 & 0 & 0 & 0 & 0 & 0 & 226 \\
\hline 8/14/04 & 7.6 & 7.0 & 12.8 & 12.6 & 7.8 & 7.4 & 14.2 & 3.8 & 5.4 & 227 \\
\hline $8 / 15 / 04$ & 1.4 & 1.2 & 0 & 0 & 0 & 0 & 0 & 0 & 1.0 & 228 \\
\hline $8 / 16 / 04$ & 0 & 0 & 0.2 & 0.2 & 0 & 0 & 0 & 0 & 0 & 229 \\
\hline $8 / 17 / 04$ & 0 & 0 & 0.4 & 0.4 & 0.2 & 0 & 0 & 0 & 13.6 & 230 \\
\hline 8/18/04 & 0 & 0 & 0 & 0 & 12.8 & 13.6 & 3.0 & 1.6 & 12.0 & 231 \\
\hline 8/19/04 & 8.0 & 6.4 & 9.0 & 10.2 & 4.0 & 3.8 & 4.4 & 2.0 & 0 & 232 \\
\hline $8 / 20 / 04$ & 0.8 & 0.8 & 23.6 & 25.8 & 8.2 & 8.2 & 0.4 & 1.2 & 0.4 & 233 \\
\hline $8 / 21 / 04$ & 0 & 0 & 1.4 & 1.2 & 0.2 & 0 & 0 & 0 & 1.0 & 234 \\
\hline $8 / 22 / 04$ & 0 & 0 & 0 & 0 & 0 & 0 & 0 & 0 & 0 & 235 \\
\hline $8 / 23 / 04$ & 0 & 0 & 0 & 0 & 0 & 0 & 0 & 0 & 0 & 236 \\
\hline 8/24/04 & 0 & 0 & 0 & 0 & 0 & 0 & 0 & 0 & 0 & 237 \\
\hline $8 / 25 / 04$ & 0 & 0 & 0 & 0 & 0 & 0 & 0 & 0 & 0 & 238 \\
\hline $8 / 26 / 04$ & 0 & 0 & 0 & 0 & 0 & 0 & 0 & 0 & 0 & 239 \\
\hline $8 / 27 / 04$ & 0 & 0 & 0 & 0 & 0 & 0 & 0 & 0 & 0 & 240 \\
\hline
\end{tabular}




\begin{tabular}{|c|c|c|c|c|c|c|c|c|c|c|}
\hline 8/28/04 & 0 & 0 & 0 & 0 & 0 & 0 & 0 & 0 & 0 & 241 \\
\hline $8 / 29 / 04$ & 0 & 0 & 0 & 0 & 0 & 0 & 0 & 0 & 0 & 242 \\
\hline $8 / 30 / 04$ & 0 & 0 & 0 & 0 & 0 & 0 & 0 & 0 & 0 & 243 \\
\hline $8 / 31 / 04$ & 0 & 0 & 0 & 0 & 0 & 0 & 0 & 0 & 0 & 244 \\
\hline $9 / 1 / 04$ & 0 & 0 & 0 & 0 & 0 & 0 & 0 & 0 & 0 & 245 \\
\hline $9 / 2 / 04$ & 0 & 0 & 0 & 0 & 0 & 0 & 0 & 0 & 0 & 246 \\
\hline $9 / 3 / 04$ & 0.8 & 0.8 & 4.2 & 4.8 & 1.4 & 1.2 & 3.2 & 0 & 0 & 247 \\
\hline $9 / 4 / 04$ & 0 & 0 & 0.4 & 0.2 & 0.2 & 0.2 & 0.2 & 0 & 0 & 248 \\
\hline $9 / 5 / 04$ & 0 & 0 & 0 & 0 & 0 & 0 & 0 & 0 & 0 & 249 \\
\hline $9 / 6 / 04$ & 0 & 0 & 0 & 0 & 0 & 0 & 0 & 0 & 0 & 250 \\
\hline $9 / 7 / 04$ & 0 & 0 & 0 & 0 & 0 & 0 & 0 & 0 & 0 & 251 \\
\hline $9 / 8 / 04$ & 0 & 0 & 0 & 0 & 0 & 0 & 0 & 0 & 0 & 252 \\
\hline $9 / 9 / 04$ & 0.2 & 0 & 0 & 0 & 0.4 & 0.2 & 0.6 & 2.8 & 0 & 253 \\
\hline $9 / 10 / 04$ & 3.0 & 2.8 & 0 & 0 & 0 & 0 & 0 & 0 & 0 & 254 \\
\hline $9 / 11 / 04$ & 0.4 & 0.6 & 0.4 & 0.2 & 0 & 0 & 0 & 0 & N/A & 255 \\
\hline $9 / 12 / 04$ & 0 & 0 & 0 & 0 & 0.2 & 0.2 & 0 & 0 & $\mathrm{~N} / \mathrm{A}$ & 256 \\
\hline $9 / 13 / 04$ & 0 & 0 & 0 & 0 & 0 & 0 & 0 & 0 & N/A & 257 \\
\hline $9 / 14 / 04$ & 0 & 0 & 0 & 0 & 0 & 0 & 0 & 0 & N/A & 258 \\
\hline 9/15/04 & 0 & 0 & 0 & 0 & 0 & 0 & 0 & 0 & N/A & 259 \\
\hline 9/16/04 & 0 & 0 & 0 & 0 & 0 & 0 & 0 & 0 & $\mathrm{~N} / \mathrm{A}$ & 260 \\
\hline $9 / 17 / 04$ & 0 & 0 & 0 & 0 & 0 & 0 & 0 & 0 & N/A & 261 \\
\hline 9/18/04 & 2.0 & 1.8 & 3.4 & 3.4 & 3.2 & 3.0 & 4.2 & 1.2 & N/A & 262 \\
\hline 9/19/04 & 35.8 & 35.2 & 49.0 & 48.0 & 21.0 & 20.2 & 20.8 & 29.4 & N/A & 263 \\
\hline 9/20/04 & 0 & 0 & 0 & 0 & 0 & 0 & 0 & 0 & N/A & 264 \\
\hline $9 / 21 / 04$ & 0 & 0 & 0 & 0 & 0 & 0 & 0 & 0 & N/A & 265 \\
\hline $9 / 22 / 04$ & 0 & 0 & 0 & 0 & 0 & 0 & 0 & 0 & $\mathrm{~N} / \mathrm{A}$ & 266 \\
\hline $9 / 23 / 04$ & 0 & 0 & 0 & 0 & 0 & 0 & 0 & 0 & $\mathrm{~N} / \mathrm{A}$ & 267 \\
\hline $9 / 24 / 04$ & 0 & 0 & 0 & 0 & 0 & 0 & 0 & 0 & N/A & 268 \\
\hline $9 / 25 / 04$ & 0 & 0 & 0 & 0 & 0 & 0 & 0 & 0 & N/A & 269 \\
\hline $9 / 26 / 04$ & 0 & 0 & 0 & 0 & 0 & 0 & 0 & 0 & $\mathrm{~N} / \mathrm{A}$ & 270 \\
\hline $9 / 27 / 04$ & 0 & 0 & 0 & 0 & 0 & 0 & 0 & 0 & N/A & 271 \\
\hline $9 / 28 / 04$ & 2.8 & 2.8 & 2.8 & 3.0 & 2.2 & 2.0 & 3.2 & 3.6 & N/A & 272 \\
\hline 9/29/04 & 12.0 & 11.6 & 4.8 & 5.2 & 5.6 & 4.8 & 5.2 & 3.0 & $\mathrm{~N} / \mathrm{A}$ & 273 \\
\hline 9/30/04 & 0 & 0 & 0 & 0 & 0 & 0 & 0 & 0 & N/A & 274 \\
\hline $10 / 1 / 04$ & 0 & 0 & 0 & 0 & 0 & 0 & 0 & 0 & N/A & 275 \\
\hline $10 / 2 / 04$ & 0 & 0 & 0 & 0 & 0 & 0 & 0 & 0 & $\mathrm{~N} / \mathrm{A}$ & 276 \\
\hline $10 / 3 / 04$ & 0 & 0 & 0 & 0 & 0 & 0 & 0 & 0 & $\mathrm{~N} / \mathrm{A}$ & 277 \\
\hline $10 / 4 / 04$ & 0.2 & 0 & 0 & 0 & 0 & 0 & 0 & 0 & N/A & 278 \\
\hline $10 / 5 / 04$ & 0.2 & 0.2 & 0.4 & 0.4 & 0 & 0 & 0 & 0 & N/A & 279 \\
\hline $10 / 6 / 04$ & 0 & 0 & 0 & 0 & 0 & 0 & 0 & 0 & $\mathrm{~N} / \mathrm{A}$ & 280 \\
\hline $10 / 7 / 04$ & 0 & 0 & 0 & 0 & 0 & 0 & 0 & 0 & N/A & 281 \\
\hline $10 / 8 / 04$ & 0 & 0 & 0 & 0 & 0 & 0 & 0 & 0 & N/A & 282 \\
\hline $10 / 9 / 04$ & 0 & 0 & 0 & 0 & 0 & 0 & 0 & 0 & N/A & 283 \\
\hline
\end{tabular}




\begin{tabular}{|c|c|c|c|c|c|c|c|c|c|c|}
\hline $10 / 10 / 04$ & 0.6 & 0.6 & 0 & 0 & 0 & 0 & 0 & 0 & $\mathrm{~N} / \mathrm{A}$ & 284 \\
\hline $10 / 11 / 04$ & 0 & 0 & 5.2 & 6.0 & 0 & 0 & 0 & 2.2 & N/A & 285 \\
\hline $10 / 12 / 04$ & 0 & 0 & 0 & 0 & 0 & 0 & 0 & 0 & N/A & 286 \\
\hline $10 / 13 / 04$ & 0 & 0 & 0 & 0 & 0 & 0 & 0 & 0 & N/A & 287 \\
\hline $10 / 14 / 04$ & 0 & 0 & 0 & 0 & 0 & 0 & 0 & 0 & N/A & 288 \\
\hline $10 / 15 / 04$ & 0 & 0 & 0 & 0 & 0 & 0 & 0 & 0 & N/A & 289 \\
\hline $10 / 16 / 04$ & 0 & 0 & 0 & 0 & 0 & 0 & 0 & 0 & N/A & 290 \\
\hline $10 / 17 / 04$ & 0 & 0 & 0 & 0 & 0 & 0 & 0 & 0 & N/A & 291 \\
\hline $10 / 18 / 04$ & 0 & 0 & 0 & 0 & 0 & 0 & 0 & 0 & $\mathrm{~N} / \mathrm{A}$ & 292 \\
\hline $10 / 19 / 04$ & 0 & 0 & 0.2 & 0.2 & 0 & 0 & 0 & 0.6 & N/A & 293 \\
\hline $10 / 20 / 04$ & 0 & 0 & 0.2 & 0.2 & 0 & 0 & 0 & 8 & N/A & 294 \\
\hline $10 / 21 / 04$ & 9.4 & 9.8 & 1.6 & 1.6 & 2.2 & 2.0 & 3.0 & 30.6 & N/A & 295 \\
\hline $10 / 22 / 04$ & 0 & 0 & 0 & 0 & 0 & 0 & 0.2 & $\mathrm{~N} / \mathrm{A}$ & N/A & 296 \\
\hline $10 / 23 / 04$ & 0 & 0 & 0 & 0 & 0 & 0 & 0 & N/A & N/A & 297 \\
\hline $10 / 24 / 04$ & 0 & 0 & 0 & 0 & 0 & 0 & 0 & N/A & N/A & 298 \\
\hline $10 / 25 / 04$ & 0 & 0 & 0 & 0 & 0 & 0 & 0 & $\mathrm{~N} / \mathrm{A}$ & $\mathrm{N} / \mathrm{A}$ & 299 \\
\hline $10 / 26 / 04$ & 0 & 0 & 0 & 0 & 0 & 0 & 0 & $\mathrm{~N} / \mathrm{A}$ & N/A & 300 \\
\hline $10 / 27 / 04$ & 0.2 & 0.2 & 0 & 0.2 & 0.4 & 0.2 & 0.6 & $\mathrm{~N} / \mathrm{A}$ & N/A & 301 \\
\hline $10 / 28 / 04$ & 1.0 & 1.0 & 1.4 & 1.2 & 3.4 & 3.4 & 3.2 & $\mathrm{~N} / \mathrm{A}$ & N/A & 302 \\
\hline $10 / 29 / 04$ & 0 & 0 & 0 & 0 & 0 & 0 & 0 & N/A & N/A & 303 \\
\hline $10 / 30 / 04$ & 0 & 0 & 0 & 0 & 0 & 0 & 0 & N/A & N/A & 304 \\
\hline $10 / 31 / 04$ & 0 & 0 & 0 & 0 & 0 & 0 & 0 & N/A & N/A & 305 \\
\hline $11 / 1 / 04$ & 0 & 0 & 0 & 0 & 0 & 0 & 0 & $\mathrm{~N} / \mathrm{A}$ & N/A & 306 \\
\hline $11 / 2 / 04$ & 0 & 0 & 0 & 0 & 0 & 0 & 0 & $\mathrm{~N} / \mathrm{A}$ & N/A & 307 \\
\hline $11 / 3 / 04$ & 0 & 0 & 0 & 0 & 0 & 0 & 0 & $\mathrm{~N} / \mathrm{A}$ & N/A & 308 \\
\hline $11 / 4 / 04$ & 0 & 0 & 0 & 0 & 0 & 0 & 0 & N/A & N/A & 309 \\
\hline $11 / 5 / 04$ & 0 & 0 & 0 & 0 & 0 & 0 & 0 & N/A & N/A & 310 \\
\hline $11 / 6 / 04$ & 0 & 0 & 0 & 0 & 0 & 0 & 0 & N/A & N/A & 311 \\
\hline $11 / 7 / 04$ & 0 & 1.8 & 1.0 & 0 & 0.2 & 0.2 & 0.2 & $\mathrm{~N} / \mathrm{A}$ & N/A & 312 \\
\hline $11 / 8 / 04$ & 7.6 & 7.6 & 17.0 & 17.8 & 17.4 & 16.8 & 16.4 & $\mathrm{~N} / \mathrm{A}$ & N/A & 313 \\
\hline $11 / 9 / 04$ & 1.4 & 1.2 & 0 & 0 & 0 & 0 & 0 & $\mathrm{~N} / \mathrm{A}$ & N/A & 314 \\
\hline $11 / 10 / 04$ & 0 & 0 & 0 & 0 & 0 & 0 & 0 & $\mathrm{~N} / \mathrm{A}$ & N/A & 315 \\
\hline $11 / 11 / 04$ & 0 & 0 & 0 & 0 & 0 & 0 & 0 & $\mathrm{~N} / \mathrm{A}$ & N/A & 316 \\
\hline $11 / 12 / 04$ & 18.8 & 19.0 & 3.4 & 3.2 & 7.6 & 8 & 10.0 & N/A & N/A & 317 \\
\hline $11 / 13 / 04$ & 0 & 0.2 & 1.0 & 1.0 & 0 & 0 & 0.2 & $\mathrm{~N} / \mathrm{A}$ & N/A & 318 \\
\hline $11 / 14 / 04$ & 0 & 0 & 0.8 & 1.0 & 0 & 0 & 0 & $\mathrm{~N} / \mathrm{A}$ & N/A & 319 \\
\hline $11 / 15 / 04$ & 0 & 0 & 0 & 0 & 0 & 0 & 0 & $\mathrm{~N} / \mathrm{A}$ & N/A & 320 \\
\hline $11 / 16 / 04$ & 0 & 0 & 0 & 0 & 0 & 0 & 0 & $\mathrm{~N} / \mathrm{A}$ & N/A & 321 \\
\hline $11 / 17 / 04$ & 0 & 0 & $\mathrm{~N} / \mathrm{A}$ & 0 & 0 & 0 & 0 & N/A & N/A & 322 \\
\hline $11 / 18 / 04$ & 0 & 0 & $\mathrm{~N} / \mathrm{A}$ & 0 & 0 & 0 & 0 & $\mathrm{~N} / \mathrm{A}$ & N/A & 323 \\
\hline $11 / 19 / 04$ & 0 & 0 & $\mathrm{~N} / \mathrm{A}$ & 0 & 0 & 0 & 0 & $\mathrm{~N} / \mathrm{A}$ & N/A & 324 \\
\hline $11 / 20 / 04$ & 2.6 & 2.6 & $\mathrm{~N} / \mathrm{A}$ & 8.0 & 7.2 & 7.2 & 7.4 & $\mathrm{~N} / \mathrm{A}$ & N/A & 325 \\
\hline $11 / 21 / 04$ & 24.6 & 24.0 & $\mathrm{~N} / \mathrm{A}$ & 20.4 & 6.8 & 6.8 & 8.0 & $\mathrm{~N} / \mathrm{A}$ & N/A & 326 \\
\hline
\end{tabular}




\begin{tabular}{|c|c|c|c|c|c|c|c|c|c|c|}
\hline $11 / 22 / 04$ & 4.4 & 4.2 & $\mathrm{~N} / \mathrm{A}$ & 12.2 & 6.2 & 5.6 & 6.6 & $\mathrm{~N} / \mathrm{A}$ & N/A & 327 \\
\hline $11 / 23 / 04$ & 0 & 0 & $\mathrm{~N} / \mathrm{A}$ & 0 & 0 & 0 & 0 & 0 & N/A & 328 \\
\hline $11 / 24 / 04$ & 0 & 0 & N/A & 0 & 0 & 0 & 0 & 0 & N/A & 329 \\
\hline $11 / 25 / 04$ & 0 & 0 & N/A & 0 & 0 & 0 & 0 & 0 & N/A & 330 \\
\hline $11 / 26 / 04$ & 0 & 0 & N/A & 0 & 0 & 0 & 0 & 0 & N/A & 331 \\
\hline $11 / 27 / 04$ & 0.2 & 0 & $\mathrm{~N} / \mathrm{A}$ & 1.2 & 0 & 0 & 0 & 0 & N/A & 332 \\
\hline $11 / 28 / 04$ & 0 & 0 & $\mathrm{~N} / \mathrm{A}$ & 1.2 & 0 & 0 & 0 & 0.6 & N/A & 333 \\
\hline $11 / 29 / 04$ & 0 & 0 & N/A & 0 & 0 & 0 & 0 & 0 & N/A & 334 \\
\hline $11 / 30 / 04$ & 0 & 0 & N/A & 0 & 0 & 0 & 0 & 0 & N/A & 335 \\
\hline $12 / 1 / 04$ & 0 & 0 & N/A & 0 & 0 & 0 & 0 & 0 & N/A & 336 \\
\hline $12 / 2 / 04$ & 0 & 0 & N/A & 0 & 0 & 0 & 0 & 0 & N/A & 337 \\
\hline $12 / 3 / 04$ & 0 & 0 & $\mathrm{~N} / \mathrm{A}$ & 0 & 0 & 0 & 0 & 0 & N/A & 338 \\
\hline $12 / 4 / 04$ & 0 & 0 & $\mathrm{~N} / \mathrm{A}$ & 0 & 0 & 0 & 0 & 0 & N/A & 339 \\
\hline $12 / 5 / 04$ & 0 & 0 & N/A & 1.0 & 0 & 0 & 0 & 0 & N/A & 340 \\
\hline $12 / 6 / 04$ & 0 & 0.2 & N/A & 1.2 & 0.8 & 0.8 & 0.8 & 0 & N/A & 341 \\
\hline $12 / 7 / 04$ & 0 & 0 & N/A & 0 & 1.6 & 0 & 0.2 & 3.8 & N/A & 342 \\
\hline $12 / 8 / 04$ & 0 & 0 & N/A & 0.2 & 0 & 1.4 & 0.2 & 0.6 & N/A & 343 \\
\hline $12 / 9 / 04$ & 0 & 0 & 0 & 0 & 0 & 0 & 0 & 0 & N/A & 344 \\
\hline $12 / 10 / 04$ & 0 & 0 & 0 & 0 & 0 & 0 & 0 & 0 & N/A & 345 \\
\hline $12 / 11 / 04$ & 0 & 0 & 0 & 0 & 0 & 0 & 0 & 0 & N/A & 346 \\
\hline $12 / 12 / 04$ & 0 & 0 & 0 & 0 & 0 & 0 & 0 & 0 & N/A & 347 \\
\hline $12 / 13 / 04$ & 0 & 0 & 0 & 0 & 0 & 0 & 0 & 0 & N/A & 348 \\
\hline $12 / 14 / 04$ & 0 & 0 & 0 & 0 & 0 & 0 & 0 & 0 & N/A & 349 \\
\hline $12 / 15 / 04$ & 0 & 0 & 0 & 0 & 0 & 0 & 0 & 0 & N/A & 350 \\
\hline $12 / 16 / 04$ & 0 & 0 & 0 & 0 & 0 & 0 & 0 & 0 & N/A & 351 \\
\hline $12 / 17 / 04$ & 0 & 0 & 0 & 0 & 0 & 0 & 0 & 0 & N/A & 352 \\
\hline $12 / 18 / 04$ & 0 & 0 & 0 & 0 & 0 & 0 & 0 & 0 & N/A & 353 \\
\hline $12 / 19 / 04$ & 0 & 0 & 0 & 0 & 0 & 0 & 0 & 0 & N/A & 354 \\
\hline $12 / 20 / 04$ & 0 & 0 & 0 & 0 & 0 & 0 & 0 & 0 & N/A & 355 \\
\hline $12 / 21 / 04$ & 0 & 0 & 0 & 0 & 0 & 0 & 0 & 0 & N/A & 356 \\
\hline $12 / 22 / 04$ & 0 & 0 & 0 & 0 & 0 & 0 & 0 & 0 & N/A & 357 \\
\hline $12 / 23 / 04$ & 0 & 0 & 0 & 0 & 0 & 0 & 0 & 0 & N/A & 358 \\
\hline $12 / 24 / 04$ & 0 & 0 & 0 & 0 & 0 & 0 & 0 & 0 & N/A & 359 \\
\hline $12 / 25 / 04$ & 0 & 0 & 0 & 0 & 0 & 0 & 0 & 0 & N/A & 360 \\
\hline $12 / 26 / 04$ & 0 & 0 & 0 & 0 & 0 & 0 & 0 & 0 & N/A & 361 \\
\hline $12 / 27 / 04$ & 0 & 0 & 0 & 0 & 0 & 0 & 0 & 0 & N/A & 362 \\
\hline $12 / 28 / 04$ & 0.2 & 0.2 & 1.4 & 1.6 & 0 & 0 & 0 & 0.4 & N/A & 363 \\
\hline $12 / 29 / 04$ & 20.0 & 19.4 & 28.2 & 28.6 & 14.6 & 14.4 & 17.8 & 12.4 & N/A & 364 \\
\hline $12 / 30 / 04$ & 0 & 0 & 0 & 0 & 0 & 0 & 0 & 0 & N/A & 365 \\
\hline $12 / 31 / 04$ & 0 & 0 & 0 & 0 & 0 & 0 & 0 & 0.2 & N/A & 366 \\
\hline
\end{tabular}



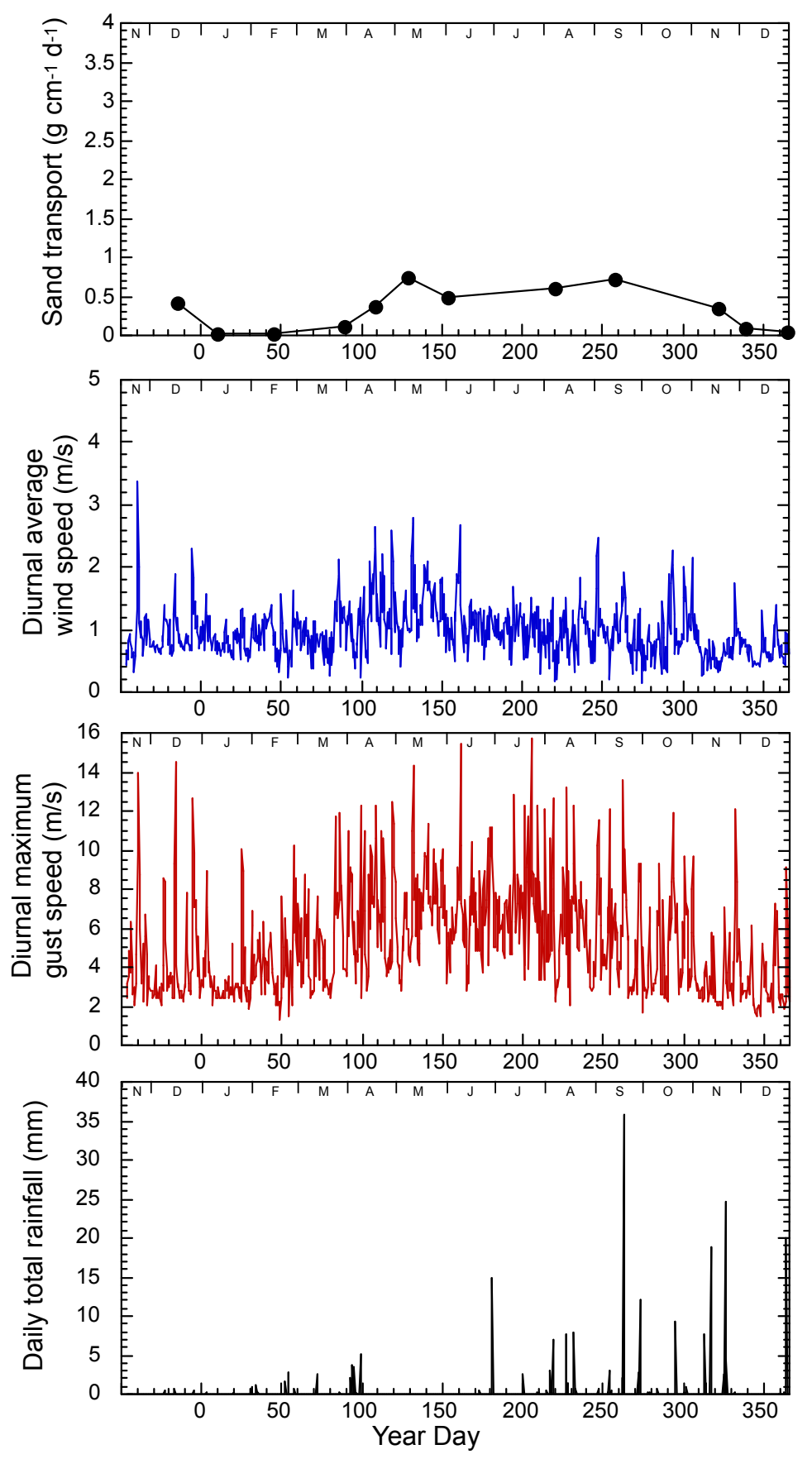

Figure 5. Sand-transport, wind, and precipitation data for the lower of the two stations deployed at 24.5 mile (Station 24.5 L). This record begins at 1814 hours on November 14, 2003, and continues through December 31, 2004 with no interruptions other than routine maintenance. For this and all other stations, sand-transport data are reported as grams per day transported between the ground surface and a height of $1 \mathrm{~m}$ (the elevation of the uppermost sand trap), normalized to a width of $1 \mathrm{~cm}$ to yield $\mathrm{g} \mathrm{cm}^{-1} \mathrm{~d}^{-1}$; sand mass collected from the four traps at each visit has been integrated over one vertical meter, and divided by the number of days since the traps had last been emptied, to obtain these values. Wind data from the upper $(2.0 \mathrm{~m})$ anemometer only are presented as diurnal averaged wind speed and diurnal maximum gust speed using daytime (0600-1800 hrs) and nighttime (1800-0600 hrs) averages of the data points collected at four-minute intervals. Rainfall is shown as daily totals summed over 24-hour periods for each day of the year. 

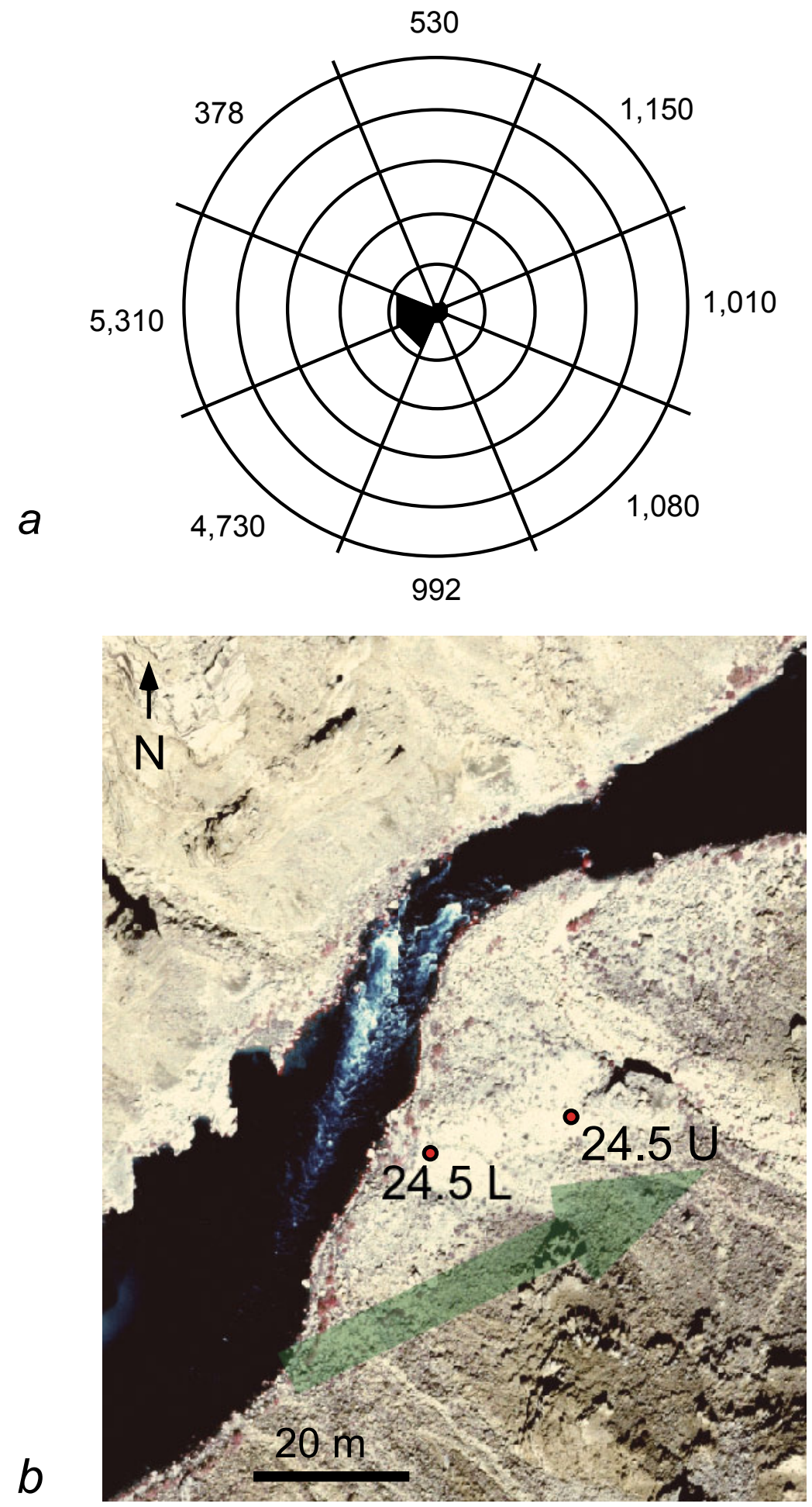

Figure 6. Potential sand transport calculated from wind data for calendar year 2004 at Station $24.5 \mathrm{~L}$. a) The rose diagram shows the total magnitude of the quantity $Q p$ for each of eight half-quadrants, indicating total potential for aeolian sediment transport from each sector. A vector sum of the data for calendar year 2004 at this station yields a net $Q p$ magnitude of $7,350 \mathrm{~m}^{3} \mathrm{~s}^{-3}$ from a direction of 244 degrees. b) aerial photograph of the 24.5-mile area. Locations of weather stations $24.5 \mathrm{~L}$ and $24.5 \mathrm{U}$ are indicated. The green arrow shows the net transport direction, from 244 degrees. 

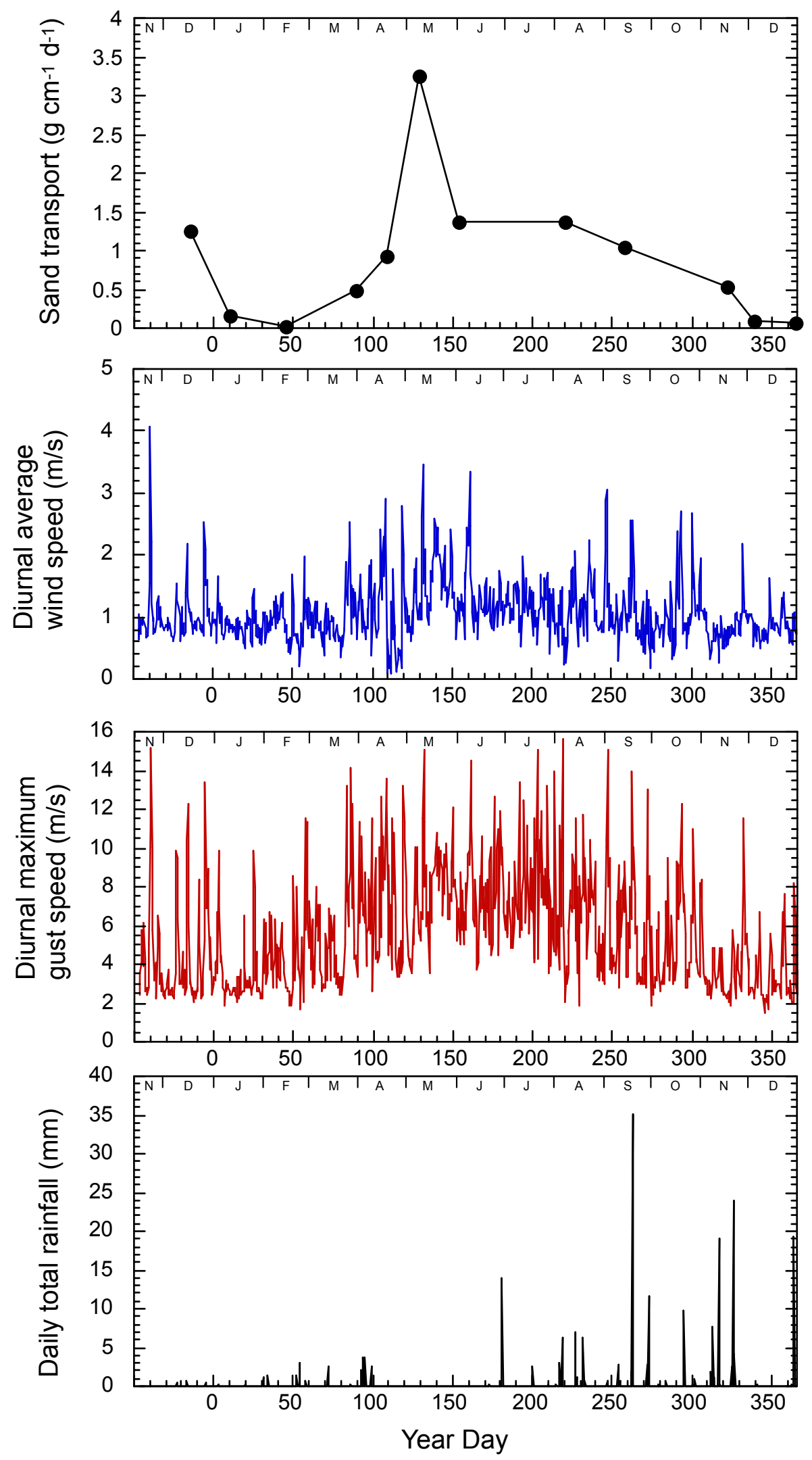

Figure 7. Sand-transport, wind, and precipitation data for the upper of the two stations deployed at 24.5 mile (Station $24.5 \mathrm{U}$ ). This record begins at 1823 hours on November 14, 2003, and continues through December 31, 2004 with no interruptions other than routine maintenance. 

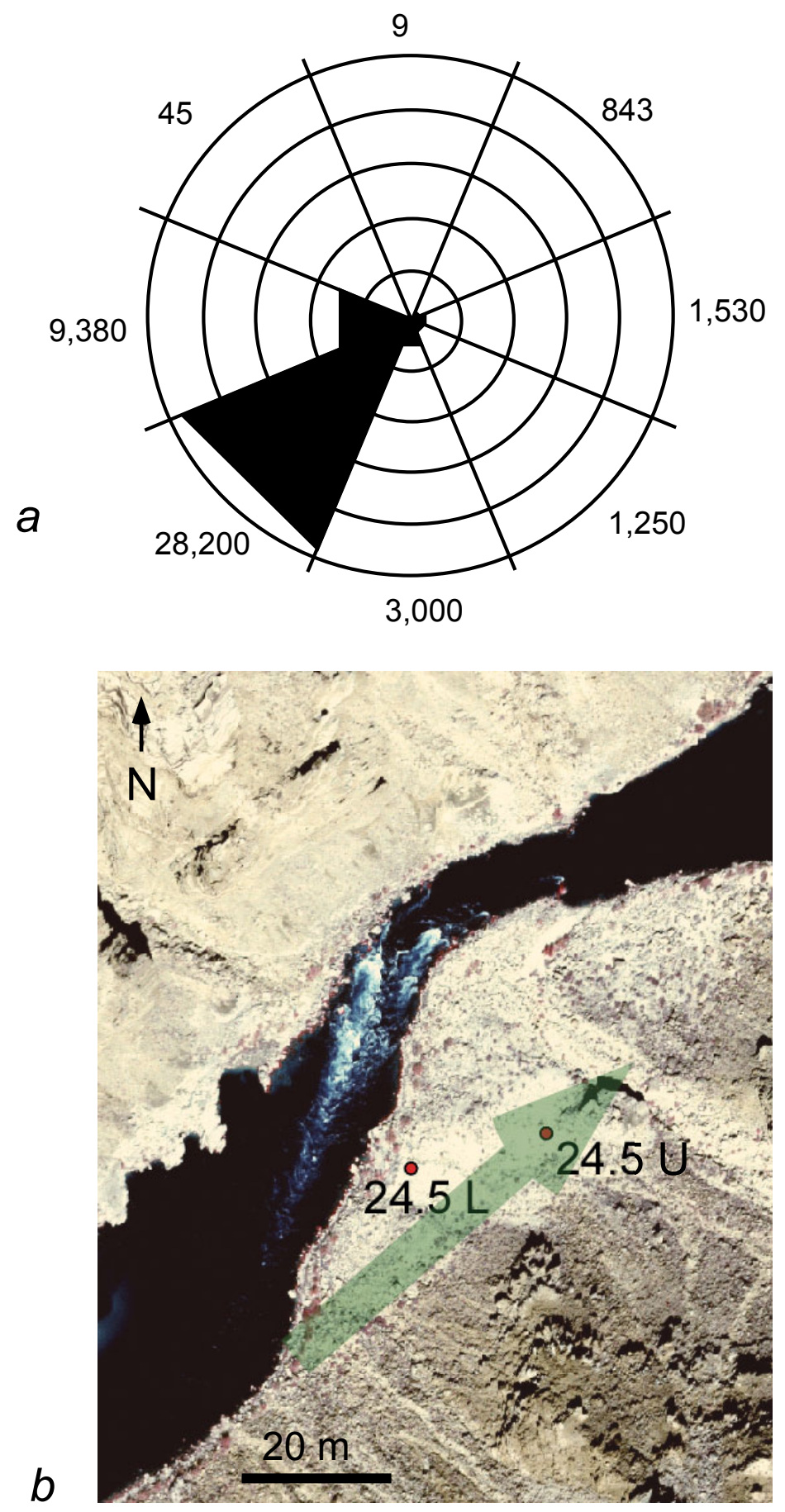

Figure 8. Potential sand transport calculated from wind data for calendar year 2004 at Station $24.5 \mathrm{U}$. a) The rose diagram shows the total magnitude of the quantity $Q_{p}$ for each of eight half-quadrants, indicating total potential for aeolian sediment transport from each sector. A vector sum of the data for calendar year 2004 at this station yields a net $Q_{p}$ magnitude of 35,800 $\mathrm{m}^{3} \mathrm{~s}^{-3}$ from a direction of 229 degrees. b) aerial photograph of the 24.5-mile area. Locations of weather stations $24.5 \mathrm{~L}$ and $24.5 \mathrm{U}$ are indicated. The green arrow shows the net transport direction, from 229 degrees. 
mobilized from a river-level sand bar near Station $24.5 \mathrm{~L}$ and transported uphill along the dune field in which Station $24.5 \mathrm{U}$ is located (fig. 6).

\section{Malgosa:}

The record for Station Mal L begins at 1614 hours on November 17, 2003, and continues through December 31, 2004. This station was intentionally disabled between 1344 hours on November 17, 2004 and 0817 hours on December 9, 2004 because the equipment had to be moved to higher elevation to avoid inundation during the November 2004 flood experiment. It was redeployed at its original location on the first post-flood river trip. Sand-transport, wind, and precipitation data for Station Mal $L$ are shown in figure 9; potential sediment transport by direction is summarized in figure 10. A vector sum of the data for calendar year 2004 at this station (excluding the time of station inactivity between November 17 and December 9) yields a net $Q p$ magnitude of 107,000 $\mathrm{m}^{3} \mathrm{~s}^{-3}$ from a direction of 132 degrees.

The record at Station Mal U begins at 1631 hours on November 17, 2003, and continues through December 31, 2004. Data collection was interrupted during several intervals by anemometer malfunctions: for 22 hours between November 21 and 23, 2003, for six hours on February 25, 2004, for a half hour on March 12, 2004, for two hours on March 13, 2004, for eighty minutes on March 20, 2004. Sand-transport, wind, and precipitation data for Station Mal L are shown in figure 11; potential sediment transport by direction is summarized in figure 12 (figs. 10a and 12a are plotted at the same scale). Rates of sediment transport measured at Station Mal U were higher than those at Station Mal L by an order of magnitude during most of the year, and by two orders of magnitude during the May windy season. Sand traps at Station Mal $U$ were emptied several times during high winds on May 10 and 11, 2004, to prevent over-filling; this accounts for the high transport rates shown in figure 11 during that time.

Sediment transport at Malgosa appears to be dominated by southeast winds that mobilize sand from the river-level sandbar near Station Mal $L$ and transport it up the dune toward Station Mal U (fig. 12). A second source of sand 



Figure 9. Sand-transport, wind, and precipitation data for the lower of the two stations deployed at Malgosa, at river mile 57.9 (Station Mal L). This record begins at 1614 hours on November 17, 2003, and continues through December 31, 2004; this station was not in operation between 1344 hours on November 17, 2004 and 0817 hours on December 9, 2004 because the equipment was moved to avoid inundation by the November 2004 flood experiment and was re-deployed on the first post-flood river trip. 



Figure 10. Potential sand transport calculated from wind data for calendar year 2004 at Station Mal L. a) The rose diagram shows the total magnitude of the quantity $Q_{p}$ for each of eight half-quadrants, indicating total potential for aeolian sediment transport from each sector. A vector sum of the data for calendar year 2004 at this station (excluding the time of station inactivity between 1344 hours on November 17 and 0817 hours on December 9) yields a net $Q_{p}$ magnitude of 107,000 $\mathrm{m}^{3} \mathrm{~s}^{-3}$ from a direction of 132 degrees. b) aerial photograph of the Malgosa (river mile 57.9) area. Locations of weather stations Mal $L$ and Mal $U$ are indicated. The green arrow shows the net transport direction, from 132 degrees. 



Figure 11. Sand-transport, wind, and precipitation data for the upper of the two stations deployed at Malgosa (Station Mal U). This record begins at 1631 hours on November 17, 2003, and continues through December 31, 2004. Data collection was interrupted during several intervals by anemometer malfunctions: for 22 hours between November 21 and 23, 2003, for six hours on February 25, 2004, for a half hour on March 12, 2004, for two hours on March 13, 2004, for eighty minutes on March 20, 2004. 



Figure 12. Potential sand transport calculated from wind data for calendar year 2004 at Station Mal $U$. a) The rose diagram shows the total magnitude of the quantity $Q_{p}$ for each of eight half-quadrants, indicating total potential for aeolian sediment transport from each sector. A vector sum of the data for calendar year 2004 at this station (excluding station inactivity in February and March that totaled $\sim 10$ hours) yields a net $Q_{p}$ magnitude of $757,000 \mathrm{~m}^{3} \mathrm{~s}^{-3}$ from a direction of 143 degrees. b) aerial photograph of the Malgosa (river mile 57.9) area. Locations of weather stations Mal $L$ and Mal $U$ are indicated. The green arrow shows the net transport direction, from 143 degrees. 
at this location is a lower-elevation sandbar on river right $\sim 120 \mathrm{~m}$ away from the dune station in which the weather stations are located; this dune field is directly upwind of the Malgosa dune field and likely serves as a sediment source to the Malgosa dunes during the spring windy season, when aeolian sand transport has been observed to cross the width of the river.

\section{Palisades:}

The record for Station Pal L begins at 1838 hours on November 18, 2003, and continues through December 31, 2004 with no interruptions other than routine maintenance. Sand-transport, wind, and precipitation data for Station Pal $L$ are shown in figure 13; potential sediment transport by direction is summarized in figure 14. A vector sum of the data for calendar year 2004 at this station yields a net $Q p$ magnitude of $38,800 \mathrm{~m}^{3} \mathrm{~s}^{-3}$ from a direction of 149 degrees. Sedimenttransport rates are very low at this station due to its location within a boulder field; an ephemeral sandbar that forms on river left immediately adjacent to the location of Station Pal L (fig. 14b) may occasionally provide a source for windblown sand, but this sandbar was not large enough to be exposed above water before the November 2004 flood experiment.

The record for Station Pal U begins at 1823 hours on November 18, 2003, and continues through December 31, 2004. Data collection was interrupted between 0832 hours on November 19, 2004 and 1304 hours on November 21, 2004 for replacement of the upper $(2.0 \mathrm{~m})$ anemometer. Sand-transport, wind, and precipitation data for Station Pal U are shown in figure 15; potential sediment transport by direction is summarized in figure 16. A vector sum of the data for calendar year 2004 at this station (excluding station inactivity between November 19 and 21) yields a net $Q p$ magnitude of $467,000 \mathrm{~m}^{3} \mathrm{~s}^{-3}$ from a direction of 155 degrees.

The dominant transport direction at Palisades is apparently toward the north-northwest, oriented upstream and slightly away from the location of the ephemeral sandbar that forms near Station Pal L. Rates of sand transport 
measured at Station Pal $\mathrm{U}$, in a relatively inactive dune field, are two to three orders of magnitude higher than those on the cobble-boulder bar at Station Pal L.

\section{Comanche:}

The record at Station Com begins at 1746 hours on April 20, 2004, and continues through December 31, 2004 with no interruptions other than routine maintenance. Sand-transport, wind, and precipitation data for this site are shown in figure 17; potential sediment transport by direction is summarized in figure 18 . A vector sum of the available data for calendar year 2004 at this station (beginning at 1746 hours on April 20) yields a net Qp magnitude of $10,200 \mathrm{~m}^{3} \mathrm{~s}^{-3}$ from a direction of 98 degrees. Net sand transport is therefore apparently directed west toward the river, and slightly upstream, in this part of the Comanche dune field.

\section{Forster:}

The record for the weather station at the mouth of Forster Canyon begins at 1517 hours on April 23, 2004, and continues through December 31, 2004. Collection of wind and precipitation data was interrupted for several weeks by malfunction of the data logger caused by a short circuit; because of this, the station was not in operation between 1448 hours on October 21, 2004 and 1458 hours on November 23, 2004. Sand-transport, wind, and precipitation data for Station For are shown in figure 19; potential sediment transport by direction is summarized in figure 20. A vector sum of the available data for calendar year 2004 at this station (beginning at 1517 hours on April 23 and excluding station inactivity between October 21 and November 23) yields a net $Q p$ magnitude of $219,000 \mathrm{~m}^{3} \mathrm{~s}^{-3}$ from a direction of 21 degrees.

High winds in late April at this site knocked over the 1.5-m-long metal sand-trap pole, resulting in the loss of sand-transport data between its initial deployment and first maintenance visit on May 17. Sand-trap data collected on August 17 and September 20, shown with red circles on figure 19, are minimum 

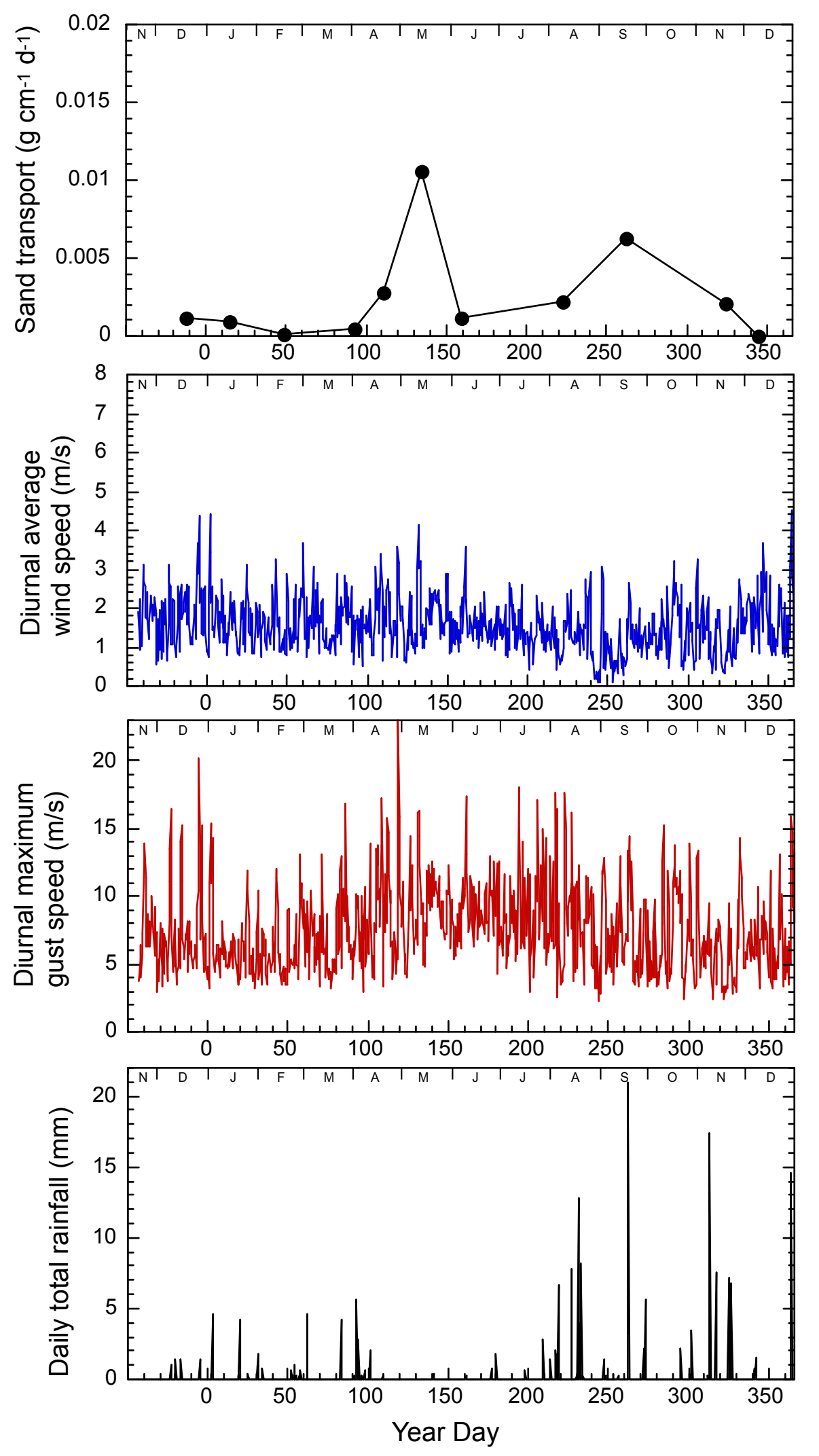

Figure 13. Sand-transport, wind, and precipitation data for the lower of the two stations deployed at Palisades, river mile 66.1 (Station Pal L). This record begins at 1838 hours on November 18, 2003, and continues through December 31, 2004 with no interruptions other than routine maintenance. 

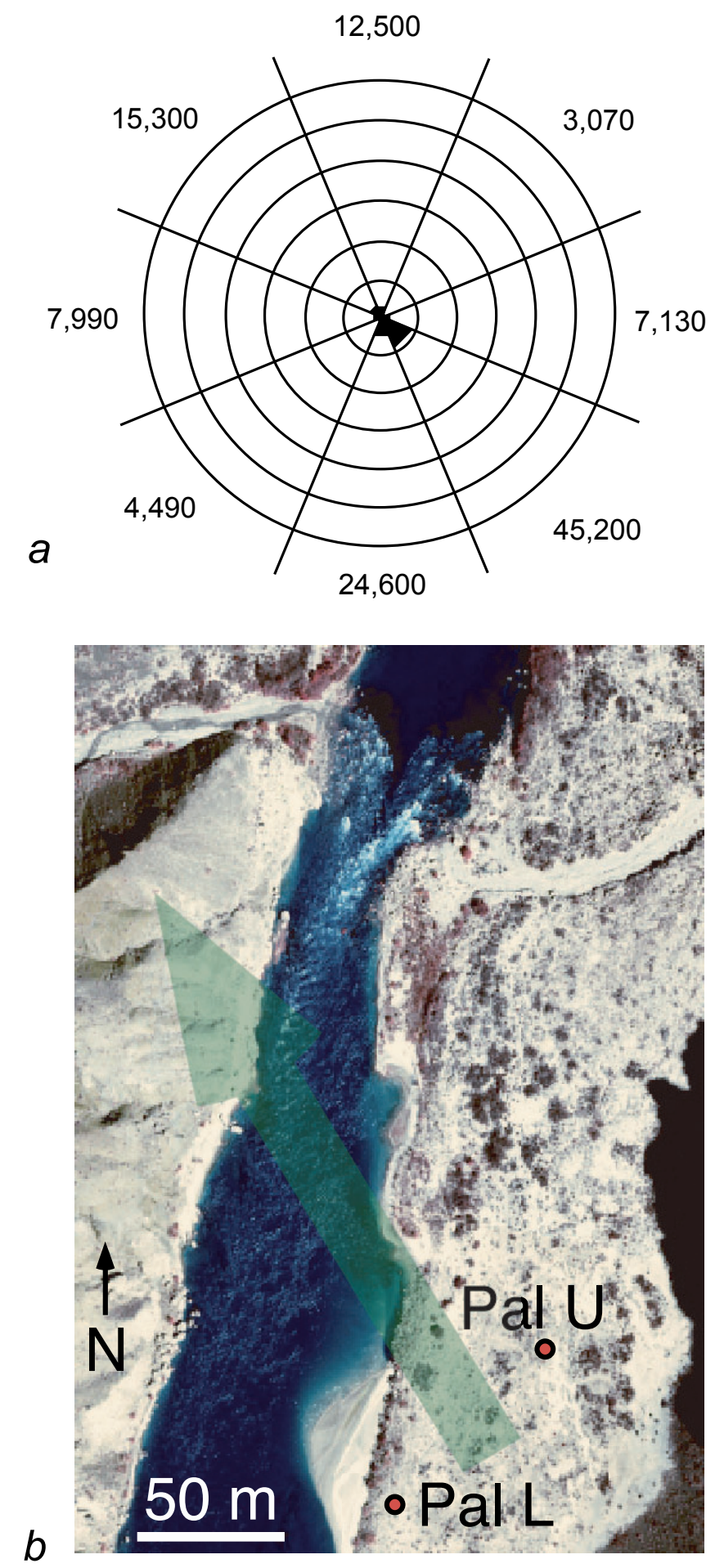

Figure 14. Potential sand transport calculated from wind data for calendar year 2004 at Station $P a l$ L. a) The rose diagram shows the total magnitude of the quantity $Q_{p}$ for each of eight half-quadrants, indicating total potential for aeolian sediment transport from each sector. A vector sum of the data for calendar year 2004 at this station yields a net $Q_{p}$ magnitude of 38,800 $\mathrm{m}^{3} \mathrm{~s}^{-3}$ from a direction of 149 degrees. b) aerial photograph of the Palisades area. Locations of weather stations Pal $L$ and Pal $U$ are indicated. The green arrow shows the net transport direction, from 149 degrees. 

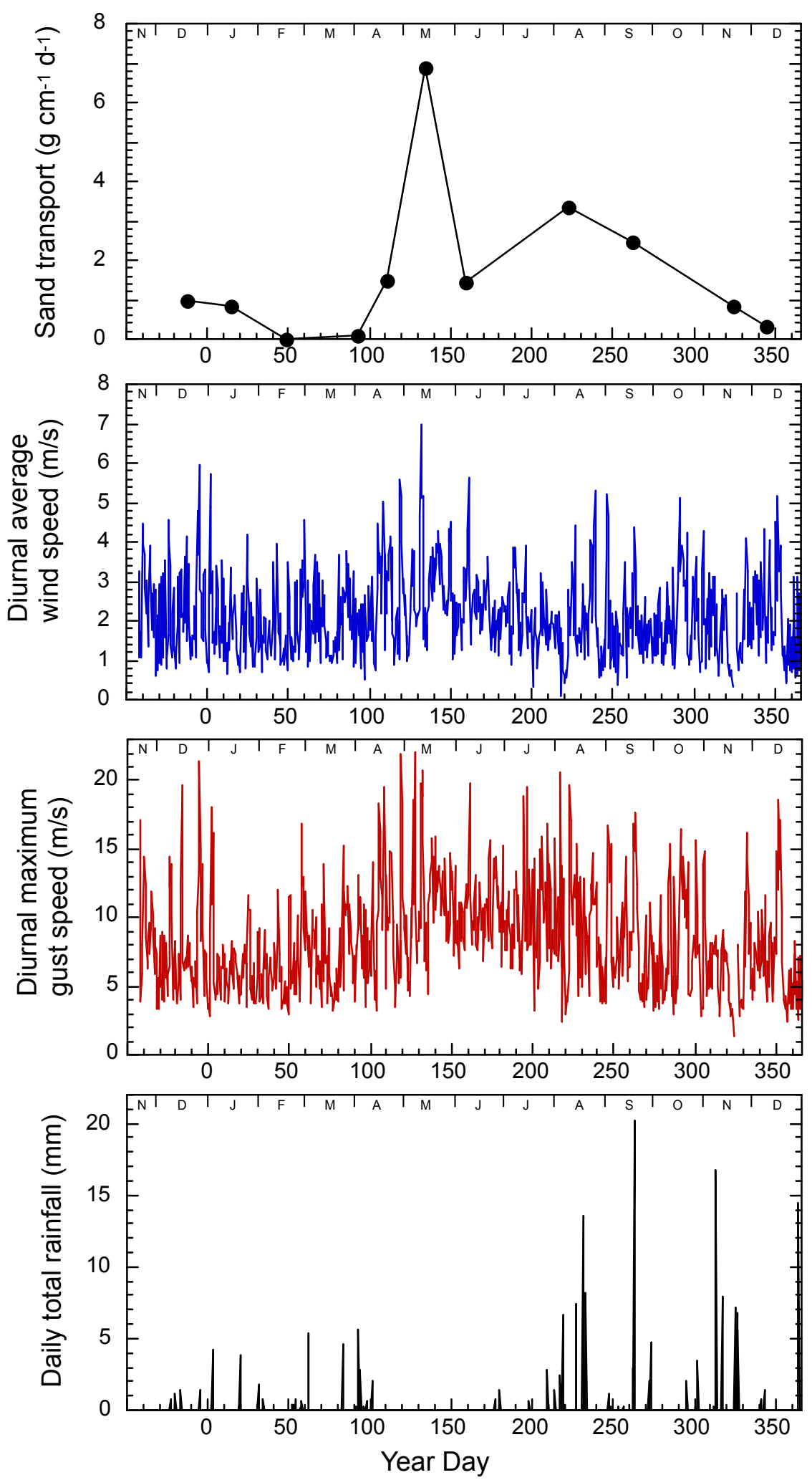

Figure 15. Sand-transport, wind, and precipitation data for the upper of the two stations deployed at Palisades, river mile 66.1 (Station Pal U). This record begins at 1823 hours on November 18, 2003, and continues through December 31, 2004. Data collection was interrupted between 0832 hours on November 19, 2004 and 1304 hours on November 21, 2004 for replacement of the upper $(2.0 \mathrm{~m})$ anemometer. 

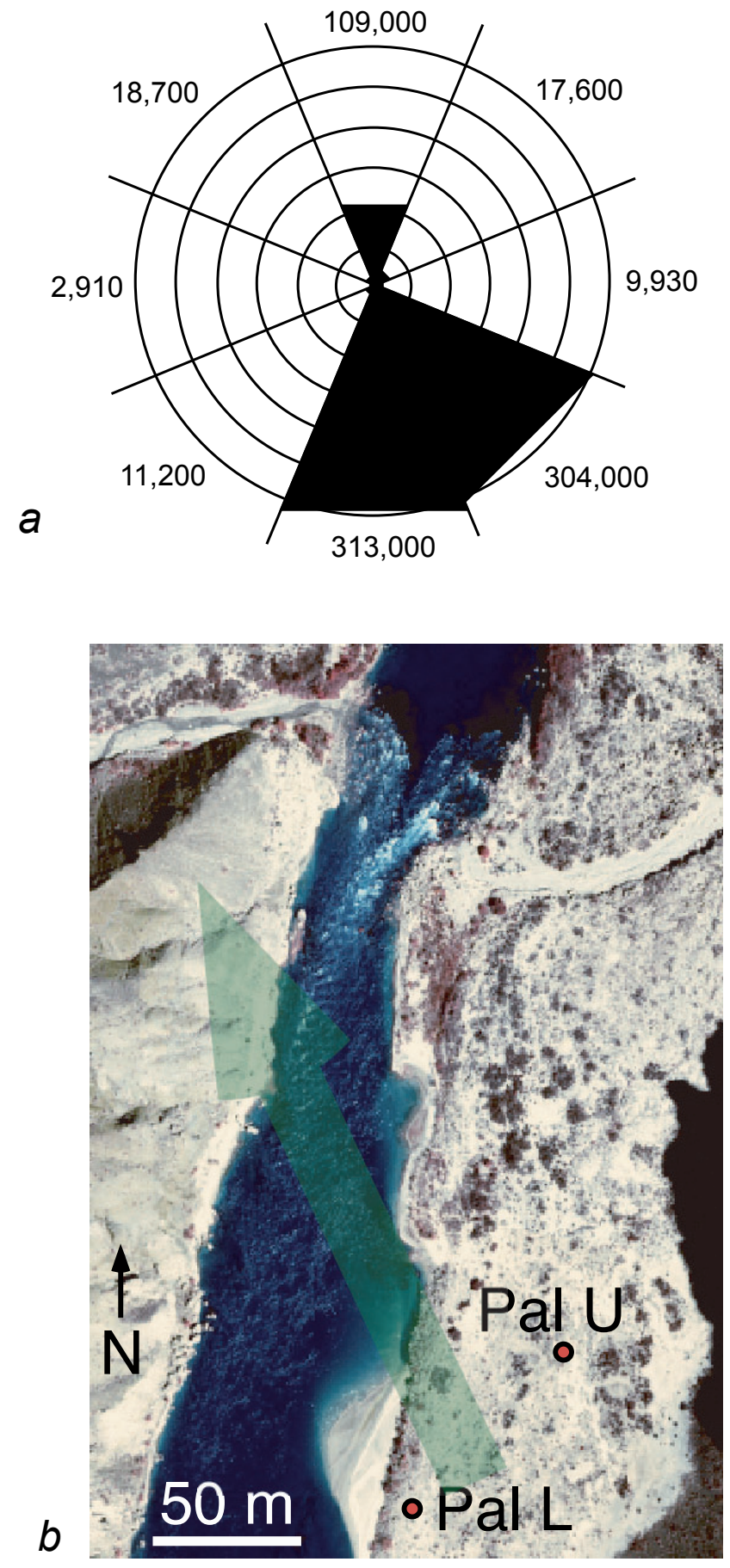

Figure 16. Potential sand transport calculated from wind data for calendar year 2004 at Station Pal U. a) The rose diagram shows the total magnitude of the quantity $Q_{p}$ for each of eight half-quadrants, indicating total potential for aeolian sediment transport from each sector. A vector sum of the data for calendar year 2004 at this station (excluding station inactivity between 0832 hours on November 19 and 1304 hours on November 21) yields a net $Q_{p}$ magnitude of $467,000 \mathrm{~m}^{3} \mathrm{~s}^{-3}$ from a direction of 155 degrees. b) aerial photograph of the Palisades area. Locations of weather stations $\mathrm{Pal} L$ and $\mathrm{Pal} U$ are indicated. The green arrow shows the net transport direction, from 155 degrees. 
estimates of transport rate only, because the lowermost trap had completely filled with sand between maintenance visits.

This site experienced high wind velocities and high rates of sediment transport throughout much of the summer and early fall; the highest wind speed recorded at Forster during the study interval occurred not during the April-May windy season but on July 16, with a gust of $29.13 \mathrm{~m} / \mathrm{s}$ (66 miles per hour). The dominant transport direction is oriented to the south-southwest, along the axis of Forster Canyon, perpendicular to the Colorado River (fig. 20).

\section{9 mile:}

The record for 202.9 mile begins at 1653 hours on April 28, 2004, and continues through November 26, 2004. Data gaps occur in this record due to multiple incidents of equipment failure. Records were interrupted at 0451 hours on September 11 due to a short circuit that damaged the rain gauge. The rain gauge was removed for repairs; the data logger with only an anemometer connected to it was launched again at 0923 hours on September 23. The logger ceased functioning at 1411 hours on November 6 and was re-launched at 1601 hours on November 24, at which time the repaired rain gauge was also reinstalled. At 1150 hours on November 26, the data logger failed again and was not re-launched until January 2005. Sand-transport, wind, and precipitation data for this site are shown in figure 21; potential sediment transport by direction is summarized in figure 22. Wind conditions at 202.9 mile are widely variable (fig. 22). Although sand traps are not used at this station, a vector sum of the available data for calendar year 2004 (excluding time when the station was not in operation) indicates net potential transport oriented upstream, with a net $Q p$ magnitude of $2,160 \mathrm{~m}^{3} \mathrm{~s}^{-3}$ from a direction of 194 degrees. 



Figure 17. Sand-transport, wind, and precipitation data for the station deployed at Comanche, river mile 68.0 (Station Com). This record begins at 1746 hours on April 20, 2004, and continues through December 31, 2004 with no interruptions other than routine maintenance. 

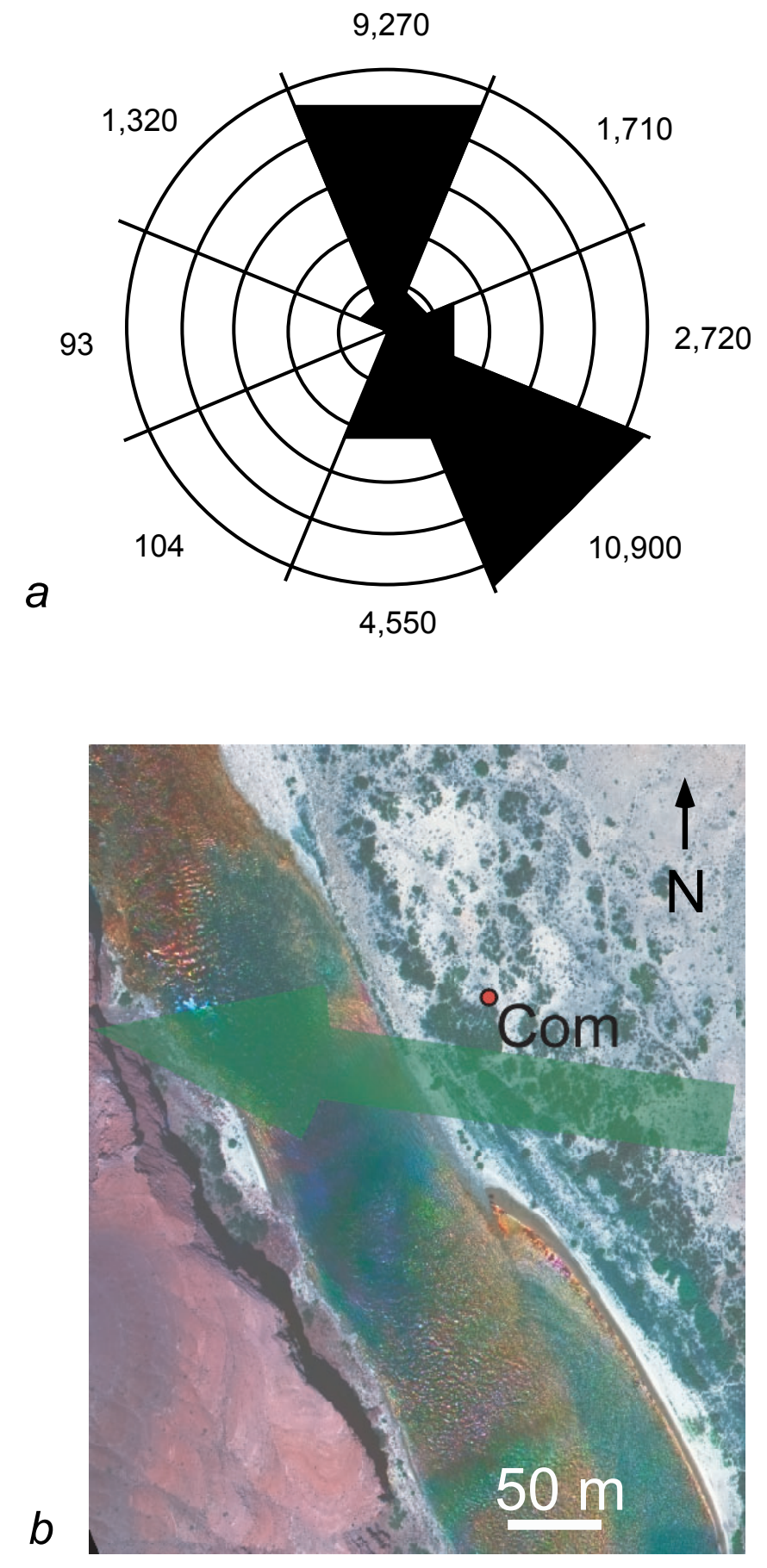

Figure 18. Potential sand transport calculated from wind data for calendar year 2004 at the Comanche weather station. a) The rose diagram shows the total magnitude of the quantity $Q_{p}$ for each of eight half-quadrants, indicating total potential for aeolian sediment transport from each sector. A vector sum of the available data for calendar year 2004 at this station (beginning at 1746 hours on April 20) yields a net $Q_{p}$ magnitude of $10,200 \mathrm{~m}^{3} \mathrm{~s}^{-3}$ from a direction of 98 degrees. b) aerial photograph of the Comanche area. The location of the weather station (Com) is indicated. The green arrow shows the net transport direction, from 98 degrees. 

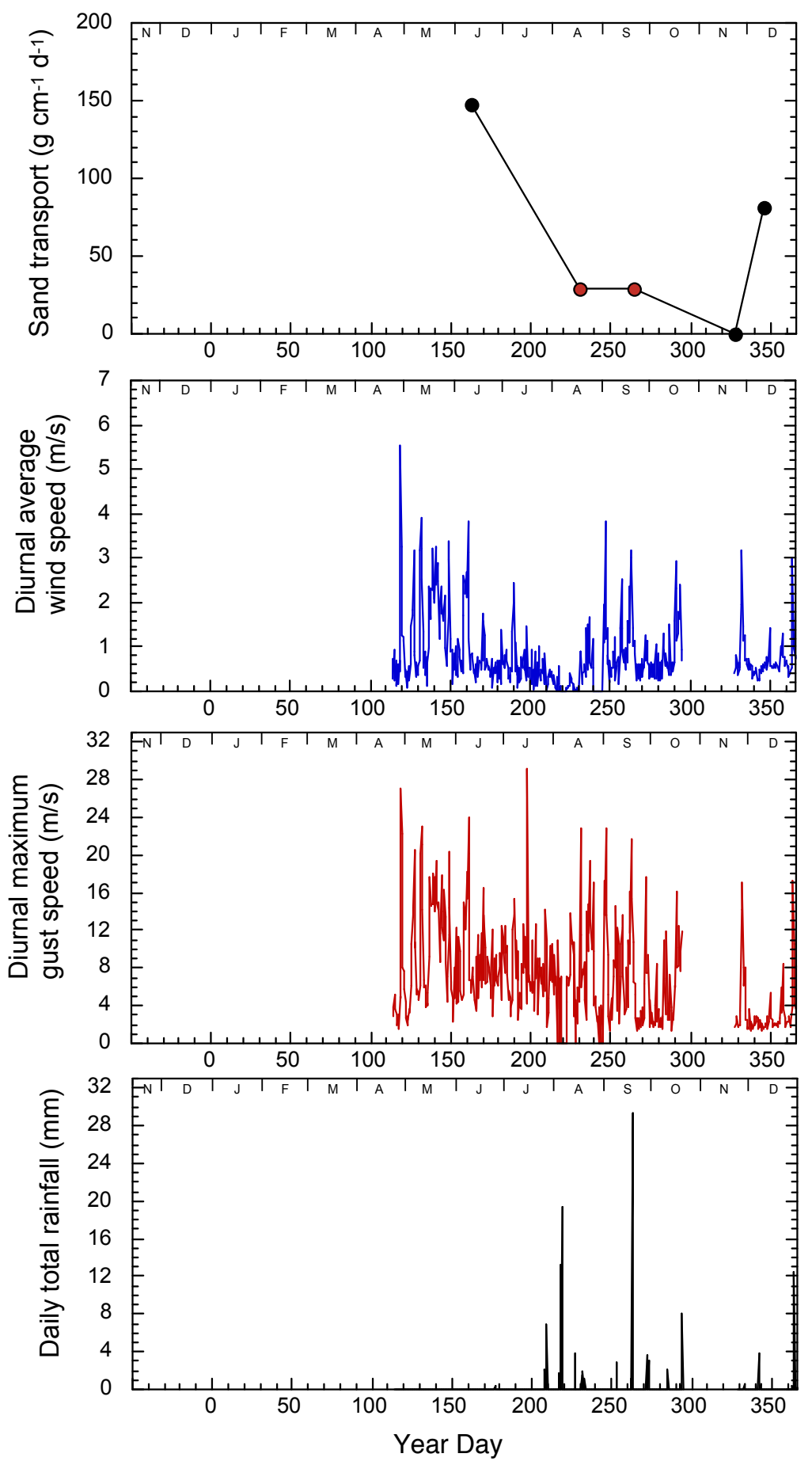

Figure 19. Sand-transport, wind, and precipitation data for the station deployed at Forster, river mile 123 (Station For). This record begins at 1517 hours on April 23, 2004, and continues through December 31, 2004. Collection of wind and precipitation data was interrupted for several weeks by malfunction of the data logger caused by a short circuit; the station was thus not in operation between 1448 hours on October 21, 2004 and 1458 hours on November 23, 2004. Sand-trap data collected on August 17 and September 20 (year days 230 and 264), shown with red circles, are minimum estimates of transport rate only, because the lowermost trap had completely filled with sand between maintenance visits. 

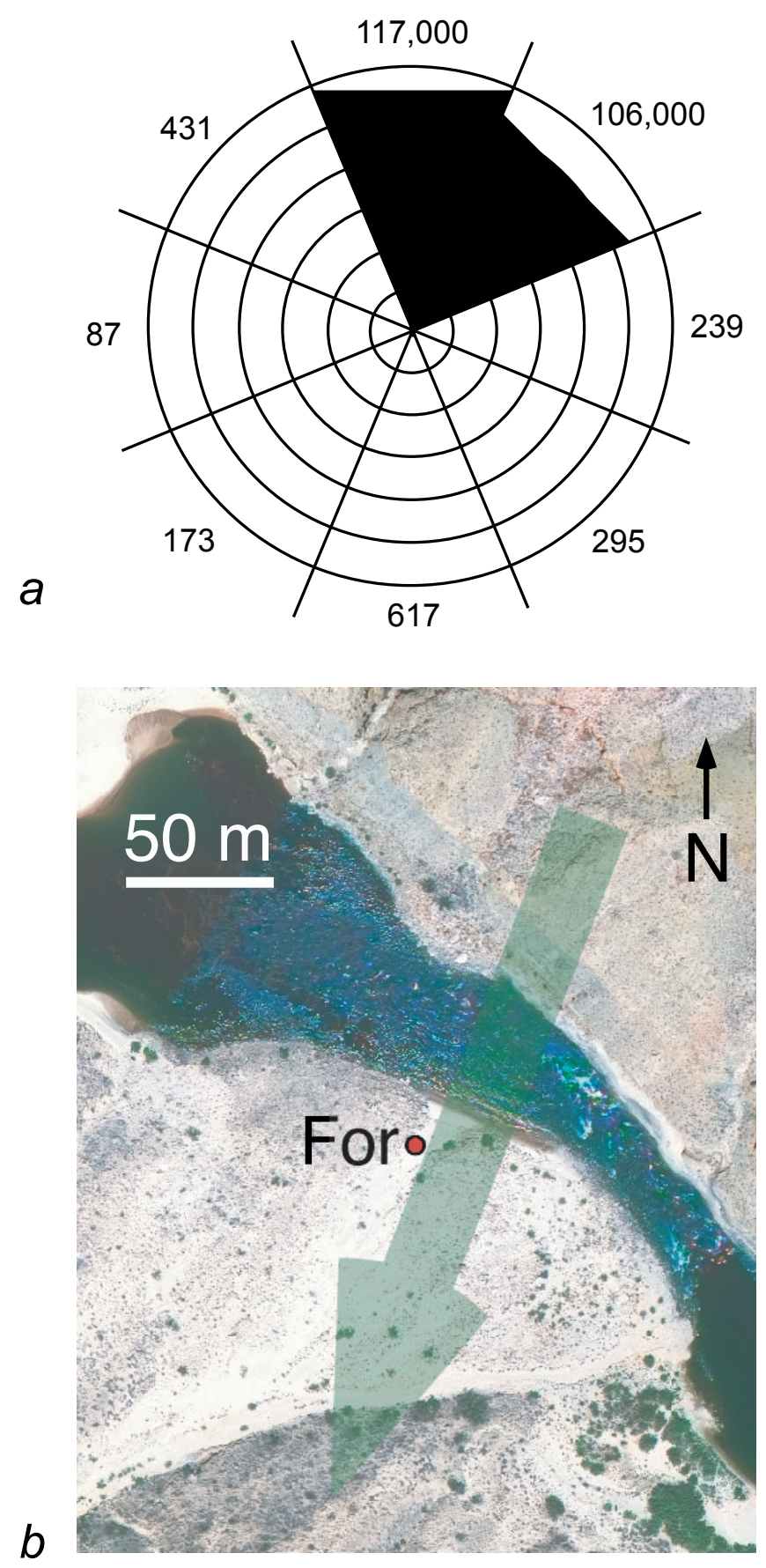

Figure 20. Potential sand transport calculated from wind data for calendar year 2004 at the Forster weather station. a) The rose diagram shows the total magnitude of the quantity $Q_{p}$ for each of eight half-quadrants, indicating total potential for aeolian sediment transport from each sector. A vector sum of the available data for calendar year 2004 at this station (beginning at 1517 hours on April 23 and excluding station inactivity between 1448 hours on October 21 and 1458 hours on November 23) yields a net Qp magnitude of $219,000 \mathrm{~m}^{3} \mathrm{~s}^{-3}$ from a direction of 21 degrees. b) aerial photograph of the Forster area. The location of the weather station (For) is indicated. The green arrow shows the net transport direction, from 21 degrees (directed up the Forster Canyon tributary). 



Figure 21. Wind and precipitation data for the station deployed at river mile 202.9 (sand traps are not in use at this station). This record begins at 1653 hours on April 28, 2004, and continues through November 26, 2004. Data gaps occur in this record due to equipment failure. Records were interrupted at 0451 hours on September 11, 2004 due to a short circuit that permanently damaged the rain gauge. The rain gauge was removed for repairs and the data logger with only an anemometer connected to it was launched again at 0923 hours on September 23. The logger ceased functioning at 1411 hours on November 6 and was re-launched at 1601 hours on November 24, at which time the repaired rain gauge was also reinstalled. At 1150 hours on November 26, the data logger failed again and was not re-launched until January 2005. 

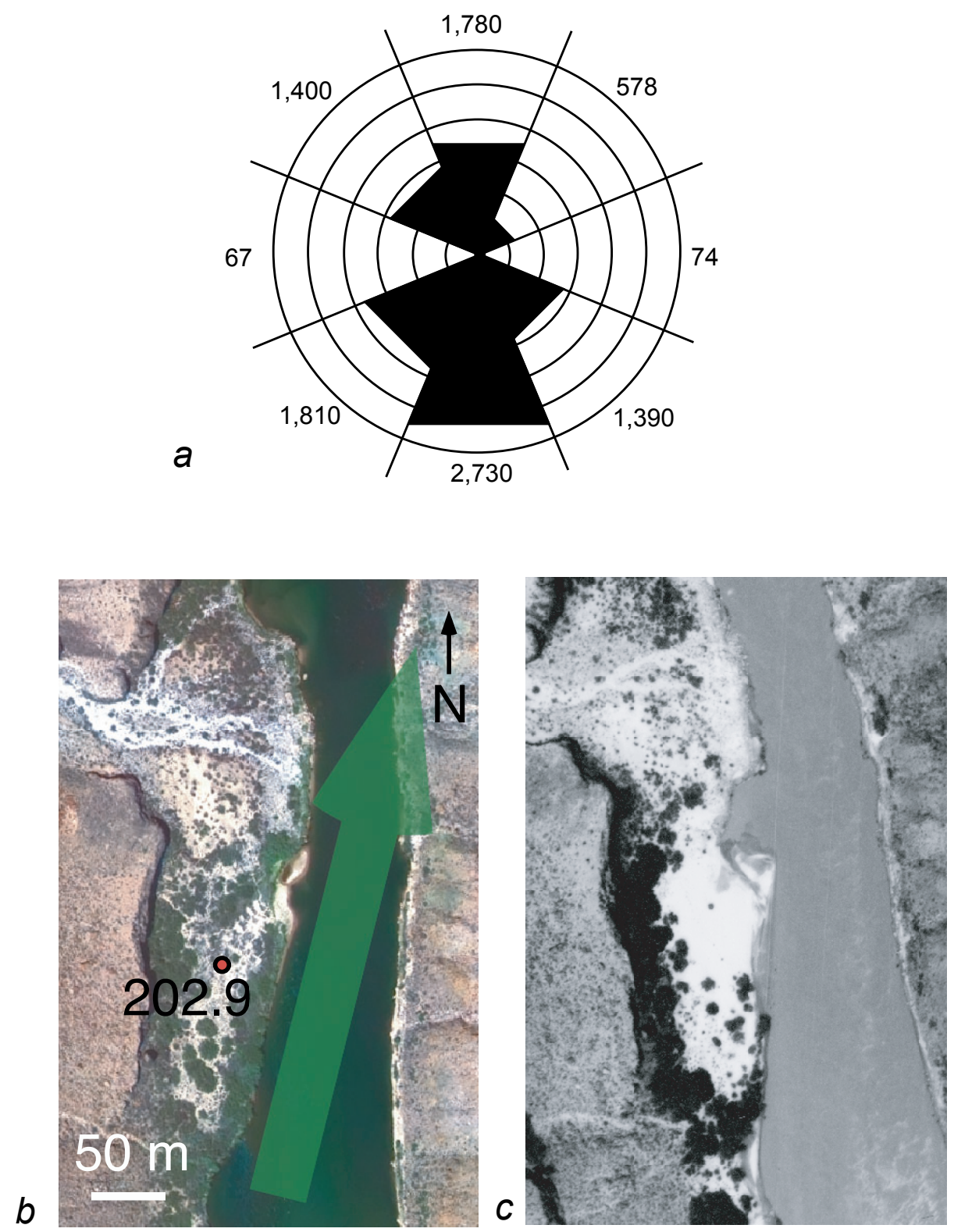

Figure 22. Potential sand transport calculated from wind data for calendar year 2004 at the 202.9-mile weather station. a) The rose diagram shows the total magnitude of the quantity $Q_{p}$ for each of eight half-quadrants, indicating total potential for aeolian sediment transport from each sector. A vector sum of the available data for calendar year 2004 at this station (beginning at 1653 hours on April 28 and excluding station inactivity between 0451 hours on September 11 and 0923 hours on September 23, and between 1411 hours on November 6 and 1601 hours on November 24; the record then ends at 1150 hours on November 26) yields a net $Q_{p}$ magnitude of 2,160 m3s-3 from a direction of 194 degrees. b) aerial photograph of the 202.9-mile area taken in 2002; the location of the weather station (202.9) is indicated. c) aerial photograph of the same area in May 1965. Vegetation encroachment on the sandbar upwind of the instrument station has been widespread in post-dam time. 


\section{DISCUSSION}

At all instrument stations, the highest wind velocities and greatest potential for sediment transport (as well as actual measured sand-transport rates) occurred during the April-May windy season. High wind velocities were also recorded at other times of year but tended to occur as isolated sets of gusts (rather than sustained high wind speed) or during individual rainstorms. When associated with rainfall during a storm event, high wind velocities generally cannot induce sediment transport because the sand is too wet to be mobilized, as in records from fall 2004. A comparison of general sediment-transport potential ( $Q p$ vector sums, tab. $2 a$ ) with that during only dry conditions (tab. $2 b$ ) reveals that although much of the wind data have been removed in order to calculate dry-sand transport potential in table $2 b$, the magnitudes of the dry $Q p$ vectors do not necessarily decrease when wind that occurred only during drysand conditions is considered. In some instances (for example, data for November 2004 at Stations Mal L and Mal U; tab. 2b) removing wind data associated with rainy weather results in higher $Q p$ values because winds opposing the dominant transport direction have been filtered out of the calculation, leaving more net transport in the dominant transport direction.

In general, data from the three locations where two weather stations are located (24.5 mile, Malgosa, and Palisades) indicate that wind velocities and aeolian sand-transport rates are uniformly higher at the higher-elevation station in each case (Stations 24.5 U, Mal U, and Pal U) compared to those measured at river level (Stations $24.5 \mathrm{~L}$, Mal L, and Pal L). This is believed to be caused largely by the effects of vegetation near the river, which reduces wind velocity and, consequently, the potential for aeolian sediment entrainment (Olson, 1958; Bressolier and Thomas, 1977; Ash and Wasson, 1983; Wasson and Nanninga, 1986; Buckley, 1987; Bauer and others, 1996). Wind can also attain higher velocity at higher elevation given the reduced interaction of air flow with 
boundaries, such as the canyon walls, as the canyon widens upward. This is not a universal rule, however, because local topography can cause major local variations in wind speed and direction.

Variations in sand-transport rates between sites are related not only to variable wind strength but also to other local factors that affect sand-entrainment potential. Sand transport may be lower at low-elevation sites near the river because residual moisture in sandbars along the river-channel margin limits entrainment of sand there by wind. Aeolian sediment transport is reduced significantly by the presence of interstitial moisture (Sarre, 1988, 1989; McKenna Neuman and Nickling, 1989; Namikas and Sherman, 1995; Wiggs and others, 2004). In Grand Canyon, sandbars inundated by the river do not have sufficient time to dry thoroughly before being inundated again the following day, in the daily flow-fluctuation regime controlled by Glen Canyon Dam.

The highest rates of sediment transport measured during this study, at Malgosa and Forster, occur in dune fields described as "active" in terms of their geomorphology: dune crests and sand shadows are well defined, with little vegetation and little to no cryptogamic soil crust. At these sites, sand-transport rates of tens of grams per centimeter per day $\left(\mathrm{g} \mathrm{cm}^{-1} \mathrm{~d}^{-1}\right)$ were common, reaching hundreds to (locally) thousands of $\mathrm{g} \mathrm{cm}^{-1} \mathrm{~d}^{-1}$ during the April-May windy season. In contrast, less active aeolian dune fields (those that have substantial vegetation cover and cryptogamic soil, such as Palisades near the Pal $U$ weather station, and Comanche) had sediment-transport rates that were an order of magnitude less than those measured at Forster and Malgosa: order $1 \mathrm{~g} \mathrm{~cm}^{-1} \mathrm{~d}^{-1}$ during the non-windy season to tens of $\mathrm{g} / \mathrm{m} / \mathrm{d}$ during the windy season. This is consistent with documentation by other studies of lower sand-transport rates over dune surfaces where cryptogamic crust is present (Leys and Eldridge, 1998; Belknap, 2001; Goossens, 2004). The dune field at 24.5 mile, which has both active and relatively inactive geomorphic zones, had transport rates that are more similar to those of Palisades and Comanche (order $1 \mathrm{~g} \mathrm{~cm}^{-1} \mathrm{~d}^{-1}$ ) than to the very active Malgosa and Forster sites. With the lowest transport rates of any sites, Station Pal $L$ typically recorded only $\sim 0.01 \mathrm{~g} \mathrm{~cm}^{-1} \mathrm{~d}^{-1}$ of aeolian sediment 
transport over the relict fluvial cobble-boulder bar on which it is located. Such low transport rates at that location are apparently caused by a low supply of available sand, and cannot be accounted for simply by lower wind speeds at Station Pal L compared to those at Station Pal U. At all stations, rates of sand transport during the April-May windy season were approximately ten times higher than at other times of year.

These data provide information on the potential for aeolian redistribution of sand from river-level fluvial deposits. This information can, in turn, be used to evaluate the potential for transport to and restorative deposition on aeolian dune fields that protect archaeological sites following the formation of new fluvial sandbars after a controlled flood. Net transport directions are locally variable; at 24.5 mile, Malgosa, and Forster, the calculated transport directions indicate net motion of sand from areas where fluvial sandbars are present toward aeolian dune fields farther from the river. At those three locations, increasing the open sand area on fluvial sandbars is expected to increase sand transport to the adjacent aeolian dune fields. However, at Palisades and Comanche the calculated dominant transport direction is oriented such that new fluvial sand deposits will not act as major sand sources for adjacent dune fields; instead, at those two sites the net transport from river-level sandbars is directed toward the Colorado River, where wind-blown sand would become entrained in the water. The 14 months of data discussed here may not be representative of wind conditions every year, though, and a longer interval of data collection will result in better defined weather patterns and potential sand-transport pathways. El Niño conditions during the 2004-2005 winter are expected to generate weather patterns, including higher rainfall, that differ from conditions measured during the year discussed here (Cayan and others, 1999; Hereford and others, 2002).

At 202.9 mile, the dominant sand transport is predicted to be directed upstream (north; fig. 22). This supports the idea that the large sandbar downstream of the weather station at that site, over which vegetation has encroached substantially since the 1960 s, formerly provided a sand source to the dune field on the debris fan upstream of the weather station. It is therefore likely 
that reduced sand-entrainment potential from the newly vegetated sandbar has affected the condition of the aeolian deposits downwind of this sandbar; reduced deposition on this dune field would exacerbate the erosive potential of rainfall and deflation by wind, both of which have affected those dunes.

\section{Effects of the November 2004 High-Flow Experiment:}

The November 2004 controlled flood release from Glen Canyon Dam, a 60 -hour steady flow of $1,160 \mathrm{~m}^{3} / \mathrm{s}\left(41,000 \mathrm{ft}^{3} / \mathrm{s}\right)$ on November 22 and 23 , occurred one year after deployment of the weather stations at 24.5 mile, Malgosa, and Palisades. These stations have therefore recorded one year of preflood aeolian sediment-transport data spanning every season, with which to compare a year of post-flood data recorded in the final, upcoming year of this study. Based on the results discussed above, the greatest potential for redistribution of new, flood-deposited sediment is expected to occur during April and May, with aeolian sand-transport rates that are predicted to be up to ten times greater than during the non-windy season.

Study sites at 24.5 mile, Malgosa, and Palisades were photographed immediately before the flood experiment began, again within one to two weeks after the flood, and a third time in March 2005. At all study sites (including Comanche, Forster, and 202.9 mile, which could not be photographed repeatedly given the field-work schedule during the flood and post-flood river trips) this flood experiment resulted in substantial new sand deposition.

From January to March, 2005, operations of Glen Canyon Dam included daily flow fluctuations between 142 and $566 \mathrm{~m}^{3} / \mathrm{s}\left(5,000-20,000 \mathrm{ft}^{3} / \mathrm{s}\right)$. These high fluctuations resulted in the removal of much of the new flood-deposited sand, as shown in figures 23,24 , and 25 . Therefore, aeolian sand-transport rates measured during the windy season beginning in April 2005 may not be substantially higher than in the previous year even with additional flood-deposited sediment, given the erosive effects of high daily fluctuations documented by photographs between January and March (figs. 23-25). At 24.5 mile and Palisades, some of the flood-deposited sand is still present; in particular, at 24.5 

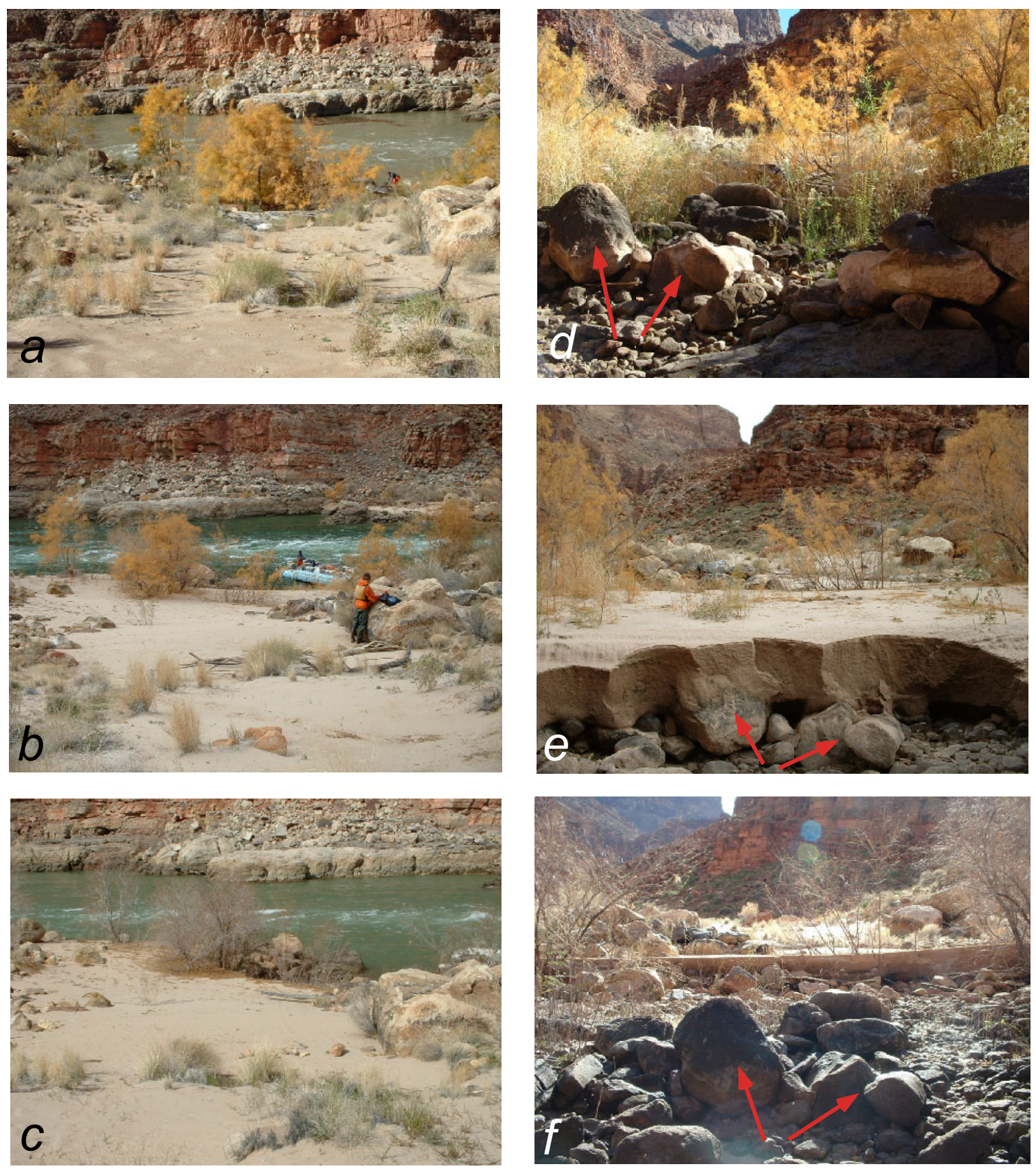

Figure 23. Photographs taken at 24.5 mile on river left (near weather station $24.5 \mathrm{~L}$ ), before $(a, d)$ and after $(b, e)$ the November 2004 flood experiment and $(c, f)$ after several months of daily flow fluctuations during which discharge ranged between 142 and $566 \mathrm{~m}^{3} / \mathrm{s}$ (5,000-20,000 ft3/s). In d, e, and f red arrows mark boulders that appear in these three pictures, for reference. The pre-flood photos (a, c) were taken on November 17, 2004, at a discharge of $226 \mathrm{~m}^{3} / \mathrm{s}(8,000 \mathrm{ft} 3 / \mathrm{s})$. Post-flood photos $(b, e)$ were taken on December 4 , 2004, at a discharge of $226 \mathrm{m3} / \mathrm{s}(8,000 \mathrm{ft} 3 / \mathrm{s})$. Photos that followed high flow fluctuations (c, f) were taken on March 8, 2005, at a discharge of approximately $226 \mathrm{~m}^{3} / \mathrm{s}(8,000 \mathrm{ft} 3 / \mathrm{s})$. New sand deposited by the November flood experiment covered vegetation, driftwood, and rocks to a thickness of up to $\sim 1 \mathrm{~m}$ (e), but had been eroded substantially by March 2005. 

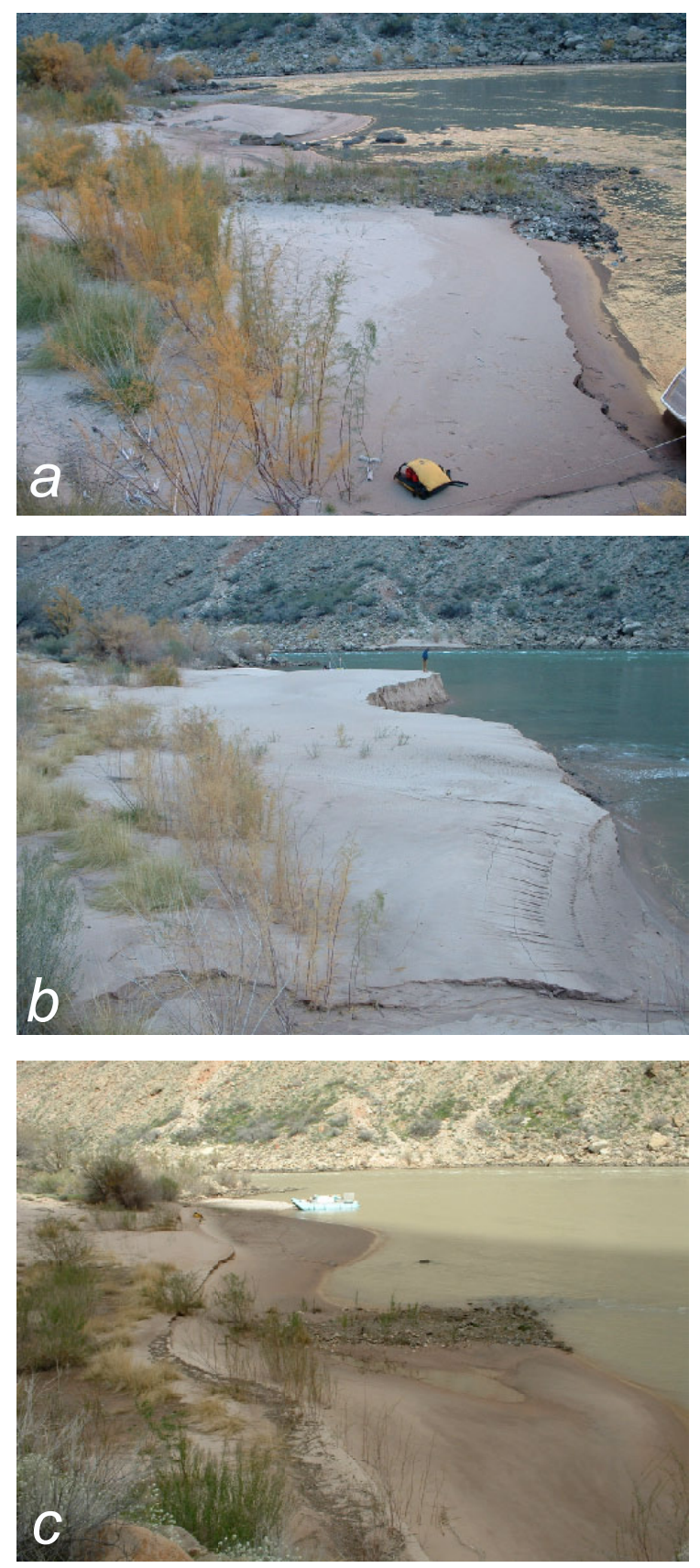

Figure 24. Photographs taken at Malgosa on river right near weather station Mal L before (a) and after (b) the November 2004 flood experiment and (c) after several months of 142-566 m3/s (5,000-20,000 ft3/s) daily flow fluctuations. The pre-flood photo (a) was taken on November 17, 2004, at a discharge of $226 \mathrm{~m}^{3} / \mathrm{s}(8,000 \mathrm{ft} / \mathrm{s})$. The post-flood photo (b) was taken on December 9, 2004, at a discharge of $\sim 226$ $\mathrm{m}^{3} / \mathrm{s}(8,000 \mathrm{ft} 3 / \mathrm{s})$. The photo that followed high flow fluctuations (c) was taken on March 13, 2005, at a discharge of $226 \mathrm{~m}^{3} / \mathrm{s}(8,000 \mathrm{ft} / \mathrm{s})$. New sand deposited at this location during the flood flow was approximately $2 \mathrm{~m}$ thick (a person standing on top of the deposit is visible for scale in the background of b). By March 2005 the flood deposit had been almost entirely removed, and the sandbar in c appears nearly identical to its pre-flood state. 

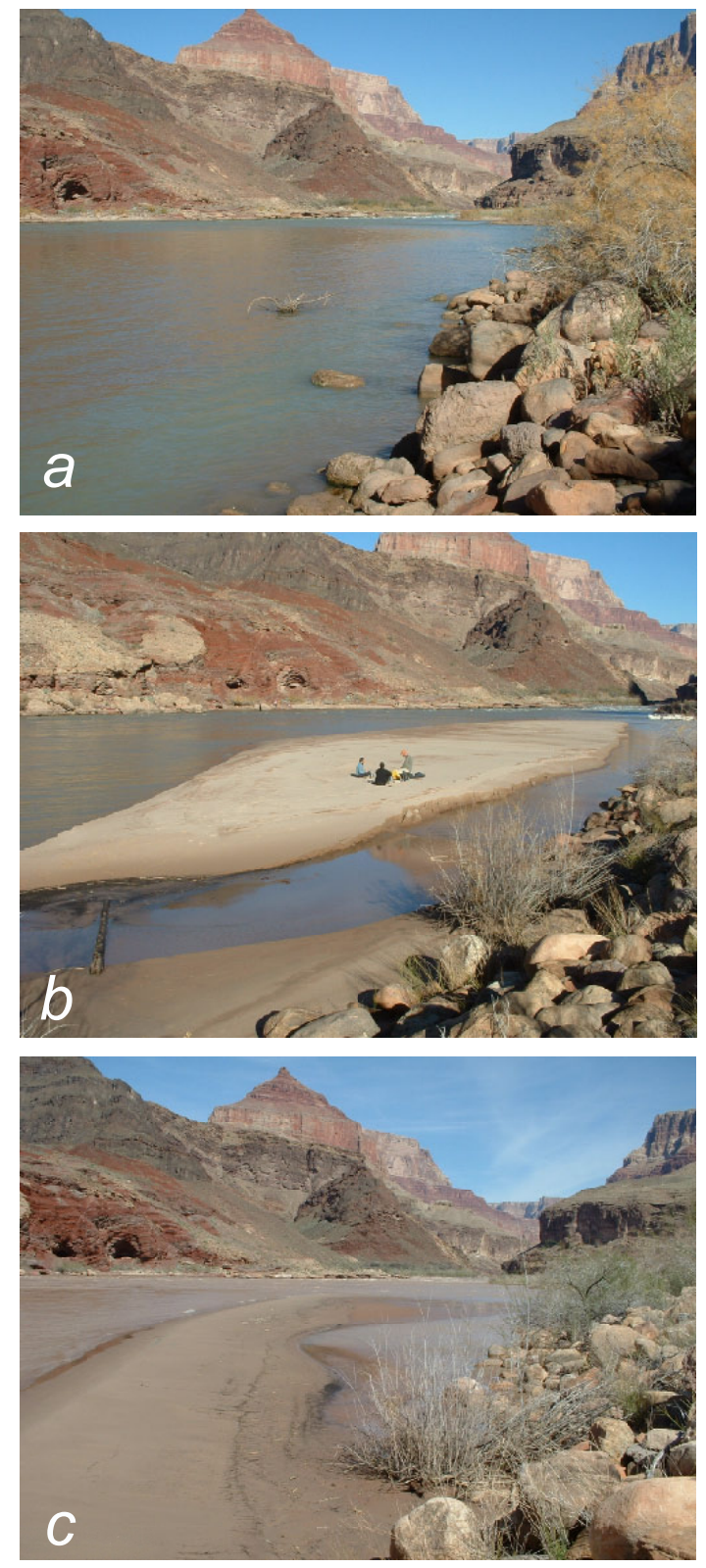

Figure 25. Photographs taken at Palisades on river left near weather station Pal $L$ before (a) and after (b) the November 2004 flood experiment and (c) after several months of 142-566 m3/s (5,000-20,000 ft3/s) daily flow fluctuations. The pre-flood photo (a) was taken on November 19, 2004, at a discharge of $226 \mathrm{~m}^{3} / \mathrm{s}(8,000$ $\left.\mathrm{ft}^{3} / \mathrm{s}\right)$. The post-flood photo (b) was taken on December 10, 2004, at a discharge of $\sim 283 \mathrm{~m}^{3} / \mathrm{s}\left(10,000 \mathrm{ft}^{3} / \mathrm{s}\right)$. The photo that followed high flow fluctuations (c) was taken on March 16, 2005, at a discharge of $\sim 425 \mathrm{~m}^{3} / \mathrm{s}\left(15,000 \mathrm{ft}^{3} / \mathrm{s}\right)$. Sediment that was deposited on this sandbar by the November flood experiment had been reworked by high daily flow fluctuations between January and March, 2005, but its location four miles downstream of the Little Colorado River (LCR) confluence indicates that additional deposition may have occurred on this sandbar during sediment-laden floods of the LCR in February, 2005. 
mile more than half of the new sand area left by the flood was located above the $566 \mathrm{~m}^{3} / \mathrm{s}\left(20,000 \mathrm{ft}^{3} / \mathrm{s}\right)$ stage, which may cause higher sand-transport rates in 2005 than in 2004 (fig. 23). At Malgosa, however, essentially all of the flooddeposited sand has been removed by the high daily flow fluctuations, and by mid-March 2005 the sandbar near Station Mal L looked almost identical to its pre-flood condition (fig. 24).

\section{CONCLUSIONS}

Data collected between November 2003 and December 2004 indicate that wind velocities and sand-transport rates were greatest during April and May 2004 (with maximum winds locally $>25 \mathrm{~m} / \mathrm{s}$, and transport rates locally $>5 \mathrm{~kg} \mathrm{~cm}^{-1} \mathrm{~d}^{-1}$ ). Dominant wind direction during strong winds varies with location, but during the April-May windy season the greatest transport potential was directed upstream in Marble Canyon (upper Grand Canyon). Such information can be used to evaluate the potential for aeolian reworking of new fluvial sand deposits, and restoration of higher-elevation aeolian deposits, following the November 2004 flood experiment. Although the 2004 flood deposited substantial quantities of new sand at all six study locations, high flow fluctuations between January and March 2005 removed much of the new sediment.

\section{ACKNOWLEDGEMENTS}

This study is supported by funding from the U.S. Bureau of Reclamation, through the Grand Canyon Monitoring and Research Center (GCMRC) and is a cooperative effort with the National Park Service (NPS). We thank the scientists and support staff of GCMRC and NPS for their collaboration in this project, as well as students and staff members from Northern Arizona University who have been essential to this project: J. Balsom, H. Chezar, J. Dierker, H. Fairley, C. 
Fritzinger, E. Fuller, R. Griffiths, S. Hueftle, K. Killoy, L. Leap, T. Melis, T. Sabol, E. Todd, D. Topping, R. Tusso, and N. Voichick. We would also like to thank the river guides who have assisted with multiple aspects of this study, in particular $\mathrm{K}$. Burnett, S. Davis, B. Dierker, J. Grissom, D. Harris, M. Piller, S. Reeder, and J. Running. Reviewers Patrick Barnard, Helen Fairley, and Dennis Fenn are thanked for their helpful comments on this manuscript. 


\section{REFERENCES}

Ash, J.E., and Wasson, R.J., 1983, Vegetation and sand mobility in the Australian desert dunefield: Zeitschrift für Geomorphologie Suppl.-Bd. 45, p. 7-25.

Bagnold, R.A., 1941, The physics of blown sand and desert dunes: London, Chapman and Hall ( $4^{\text {th }}$ edition, 1973), 265 pages.

Bauer, B.O., Davidson-Arnott, R.G.D., Nordstrom, K.F, Ollerhead, J., and Jackson, N.L., 1996, Indeterminacy in aeolian sediment transport across beaches: Journal of Coastal Research, v. 12, p. 641-653.

Belknap, J., 2001, Biological soil crusts and wind erosion, in Biological soil crusts-structure, function, and management: New York, Springer-Verlag, p. 339-347.

Beus, S.S., Carothers, S.W., and Avery, C.C., 1985, Topographic changes in fluvial terrace deposits used as campsite beaches along the Colorado River in Grand Canyon: Journal of the Arizona-Nevada Academy of Science, v. 20, p. 111-120.

Bressolier, C., and Thomas, Y.-F., 1977, Studies on wind and plant interactions on French Atlantic coastal dunes: Journal of Sedimentary Petrology, v. 47, p. 331-338.

Buckley, R., 1987, The effect of sparse vegetation on the transport of dune sand by wind: Nature v. 325, p. 426-428.

Cayan, D.R., Redmond, K.T., and Riddle, L.G., 1999, ENSO and hydrologic extremes in the western United States: Journal of Climate, v. 12, p. 2881-2893.

Fryrear, D.W., 1986, A field dust sampler: Journal of Soil and Water Conservation, v. 41, p. 117-119.

Fryrear, D.W., Stout, J.E., Hagen, L.J., and Vories, E.D., 1991, Wind erosionfield measurement and analysis: Transactions of the American Society of Agricultural Engineers, v. 34, p. 155-160. 
Goossens, D., and Offer, Z.Y., 2000, Wind tunnel and field calibration of six aeolian dust samplers: Atmospheric Environment, v. 34, p. 1043-1057.

Goossens, D., Offer, Z., and London, G., 2000, Wind tunnel and field calibration of five aeolian sand traps: Geomorphology, v. 35, p. 233-252.

Goossens, D., 2001, Calibration of aeolian sediment catchers: WEELS (Wind Erosion on European Light Soils) Final Report, section 4.2, http://www.geog.ucl.ac.uk/weels/final_report/section_4.2.pdf

Goossens, D., 2004, Effect of soil crusting on the emission and transport of winderoded sediment: field measurements on loamy sandy soil: Geomorphology, v. 58 , p. $145-160$.

Hereford, R., Webb, R.H., and Graham, S., 2002, Precipitation history of the Colorado Plateau region, 1900-2000: U.S. Geological Survey Fact Sheet 119-02.

Kaplinski, M., Hazel, J.E. Jr., and Beus, S.S., 1995, Monitoring the effects of interim flows from Glen Canyon Dam on sand bars in the Colorado River corridor, Grand Canyon National Park, Arizona: Final report to Glen Canyon Environmental Studies, Northern Arizona University, Flagstaff, Ariz., 62 pages.

Kawamura, R., 1951, Study of sand movement by wind: Tokyo, Japan, University of Tokyo, Report of the Institute of Science and Technology, v. 5, p. 95-112.

Kearsley, L.H., Schmidt, J.C., and Warren, K.D., 1994, Effects of Glen Canyon Dam on Colorado River sand deposits used as campsites in Grand Canyon National Park, USA: Regulated Rivers: Research and Management, v. 9, p. $137-149$.

Lettau, K., and Lettau, H.H., 1977, Experimental and micro-meteorological field studies of dune migration, in Lettau, H.H., and Lettau, K., eds., Exploring the World's Driest Climates: Madison, Wisc., University of Wisconsin, Institute of Environmental Science Report, v. 101, p. 110-147. 
Leys, J.F., and Eldridge, D.J., 1998, Influence of cryptogamic crust disturbance to wind erosion on sand and loam rangeland soils: Earth Surface Processes and Landforms, v. 23, p. 963-974.

McEwan, I.K., and Willets, B.B., 1993, Sand transport by wind-a review of the current conceptual model, in Pye, K., ed., The Dynamics and Environmental Context of Aeolian Sedimentary Systems: Geological Society Special Publication 72, p. 7-16.

McKenna Neuman, C., and Nickling, W.G., 1989, A theoretical and wind-tunnel investigation of the effect of capillary water on the entrainment of sediment by wind: Canadian Journal of Soil Science, v. 69, p. 79-96.

Namikas, S.L., and Sherman, D.J., 1995, A review of the effects of surface moisture content on aeolian sand transport, in Tchakerian, V., ed., Desert aeolian processes: London, Chapman and Hall, p. 269-293.

Nickling, W.G., and McKenna Neuman, C., 1997, Wind tunnel evaluation of a wedge-shaped aeolian sediment trap: Geomorphology, v. 18, p. 333-345.

Olson, J.S., 1958, Lake Michigan dune development 1-wind-velocity profiles: Journal of Geology, v. 66, p. 254-263.

Rubin, D.M., and Topping, D.J., 2001, Quantifying the relative importance of flow regulation and grain size regulation of suspended sediment transport $\square$ and tracking changes in grain size of bed sediment $\square$ : Water Resources Research, v. 37, p. 133-146.

Rubin, D.M., Topping, D.J., Schmidt, J.C., Hazel, J., Kaplinski, M., and Melis, T.S., 2002, Recent sediment studies refute Glen Canyon Dam hypothesis: EOS, Transactions of the American Geophysical Union, v. 83, p. 277-278.

Sarre, R.D., 1988, Evaluation of aeolian sand transport equations using intertidal zone measurements, Saunton Sands, England: Sedimentology, v. 35, p. 671-679.

Sarre, R.D., 1989, Aeolian sand drift from the intertidal zone on a temperate beach: potential and actual rates: Earth Surface Processes and Landforms, v. 14 , p. $247-258$. 
Schmidt, J.C., and Graf, J.B., 1987, Aggradation and degradation of alluvial sand deposits, 1965 to 1986, Colorado River, Grand Canyon National Park, Arizona: U.S. Geological Survey Open-File Report 87-555, 120 pages.

Schmidt, J.C., Topping, D.J., Grams, P.E., and Hazel, J.E., 2004, System-wide changes in the distribution of fine sediment in the Colorado River corridor between Glen Canyon Dam and Bright Angel Creek, Arizona: Final report submitted to Grand Canyon Monitoring and Research Center by the Department of Aquatic, Watershed, and Earth Resources, Utah State University, 107 pages.

Shao, Y., McTainsh, G.H., Leys, J.F., and Raupach, M.R., 1993, Efficiencies of sediment samplers for wind erosion measurements: Australian Journal of Soil Research, v. 31, p. 519-532.

Sherman, D.J., Jackson, D.W.T., Namikas, S.L., and Wang, J., 1998, Windblown sand on beaches-an evaluation of models: Geomorphology, v. 22, p. 113-133.

Sterk, G., and Raats, P.A.C., 1996, Comparison of models describing the vertical distribution of wind-eroded sediment: Soil Science Society of America Journal, v. 60, p. 1914-1919.

Stout, J.E., and Fryrear, D.W., 1989, Performance of a windblown-particle sampler: Transactions of the American Society of Agricultural Engineers, v. 32, p. 2041-2045.

Topping, D.J., Rubin, D.M., and Vierra, L.E. Jr., 2000a, Colorado River sediment transport 1. Natural sediment supply limitation and the influence of Glen Canyon Dam: Water Resources Research, v. 36, p. 515-542.

Topping, D.J., Rubin, D.M., Nelson, J.M., Kinzel III, P.J., and Corson, I.C., 2000b, Colorado River sediment transport 2. Systematic bed-elevation and grain-size effects of sand supply limitation: Water Resources Research, v. 36, p. 543-570.

Vories, E.D., and Fryrear, D.W., 1991, Vertical distribution of wind-eroded soil over a smooth, bare field: Transactions of the American Society of Agricultural Engineers, v. 34, p. 1763-1768. 
Wasson, R.J., and Nanninga, P.M., 1986, Estimating wind transport of sand on vegetated surfaces: Earth Surface Processes and Landforms, v. 11, p. 505-514.

Webb, R.H., Schmidt, J.C., Marzolf, G.R., and Valdez, R.A., eds., 1999, The controlled flood in Grand Canyon: Washington, D.C., American Geophysical Union, Geophysical Monograph 110, p. 1-21.

Wiggs, G.F.S., Baird, A.J., and Atherton, R.J., 2004, The dynamic effects of moisture on the entrainment and transport of sand by wind: Geomorphology, v. 59 , p. $13-30$.

Zobeck, T.M., and Fryrear, D.W., 1986, Chemical and physical characteristics of windblown sediment I, Quantities and physical characteristics: Transactions of the American Society of Agricultural Engineers, v. 29, p. 1032-1036.

Zobeck, T.M., Sterk, G., Funk, R., Rajot, J.L., Stout, J.E., and Van Pelt, R.S., 2003, Measurement and data analysis methods for field-scale wind erosion studies and model validation: Earth Surface Processes and Landforms, v. 28, p. 1163-1188. 